\title{
Um arcabouço generalizado para empacotamento de ramificações e outras estruturas combinatórias
}

\author{
Mario Leston Rey
}

TESE APRESENTADA

AO

Instituto DE MATEMÁticA E EstatísticA

DA

UNIVERSIDADE DE SÃo PAUlo

PARA

OBTENÇÃO DO TÍTULO

$\mathrm{DE}$

DOUTOR EM CIÊNCIAS

Programa: Doutorado em Ciência da Computação

Orientadora: Profa. Dra. Yoshiko Wakabayashi

Durante o desenvolvimento deste trabalho o autor recebeu auxílio financeiro do CNPq

São Paulo, novembro de 2012 


\section{Um arcabouço generalizado para empacotamento de ramificações e outras estruturas combinatórias}

Esta versão da tese contém as correções e alterações sugeridas pela Comissão Julgadora durante a defesa da versão original do trabalho, realizada em 22/11/2012. Uma cópia da versão original está disponível no Instituto de Matemática e Estatística da Universidade de São Paulo.

Comissão Julgadora:

- Profa. Dra. Yoshiko Wakabayashi - IME-USP

- Prof. Dr. Jose Coelho de Pina Junior - IME-USP

- Prof. Dr. Marcelo Henriques de Carvalho - UFMS

- Prof. Dr. Orlando Lee - UNICAMP

- Prof. Dr. Sóstenes Luiz Soares Lins - UFPE 
Ao meu filho, Pedro, que iluminou a minha existência e a de meus pais. Ao meu irmão, Antonio, que é a luz que mais nos faz falta. 


\section{Agradecimentos}

À Yoshiko, pela orientação, compreensão, dedicação, cuidado, atenção, apoio, encorajamento, e, ademais, pela paciência em aturar um ser caótico e intermitente como eu.

Aos professores José Coelho de Pina, Marcelo Henriques de Carvalho, Orlando Lee, e Sóstenes Lins, pelas inúmeras sugestões.

Ao Paulo Feofiloff, um sujeito ímpar, lúcido e inspirador, responsável em grande parte (queira ele ou não) pelos lampejos de qualidade (se é que existem) deste trabalho. É uma honra e alegria tê-lo como amigo.

Ao Pil, outro sujeito ímpar, que há muito tempo já deixou a categoria de amigo e tornou-se irmão, pela irmandade incondicional, por sempre me ouvir, apoiar, compreender e, principalmente, participar positivamente e decisivamente nas escolhas importantes de minha vida.

Ao meus pais - sempre presentes, responsáveis pelas minhas qualidades, mas não pelos meus defeitos - por todo apoio, dedicação, amor e por viabilizarem a realização deste trabalho. Em particular, à minha incansável mãe, que além de cuidar de mim, cuida do meu filho e do meu pai.

À minha Linda, por ser fonte de inspiração, e por todo amor, carinho, apoio, dedicação, encorajamento, e compreensão, sem os quais este trabalho não poderia ter sido realizado.

Aos professores Marcelo Rezende, Dário Alliprandini e Sílvia Boarin da ESEG, por proporcionarem um ambiente de trabalho amistoso, saudável, responsável e honesto. Ao Marcelo, em particular, por toda a amizade e apoio, tanto em questões particulares quanto nas laborais.

Ao Coelho, pelo apoio e pelas conversas.

Ao Yoshiharu, ao Carlinhos, à Cris, ao Alair, ao Finger e a Gordana, pelas conversas e sugestões.

Ao Lymber, amigo já de muitos anos, por estar presente num momento crítico.

Ao Ricardo Witte, o alemão, pela amizade incondicional.

Aos amigos do IME, Elói, Renato do Carmo, Jair, Rozante e Fábio Martinez.

Aos amigos da FIEO, Andrea Machion, Janice, Olímpio, Guardado e Fabiana. Ao Márcio Mathias e ao Sandro Verri, pela amizade e pela diversão que nossas conversas me proporcionam.

À minha família em Pontevedra, e sobretudo à minha avó, por participarem de minha vida mesmo tão distantes.

Ao meu filho, simplesmente por ser meu filho.

Finalmente, ao meu irmão, minha maior influência e fonte de inspiração. 


\section{Resumo}

REY, M. L. Um arcabouço generalizado para empacotamento de ramificações e outras estruturas combinatórias 2012. 91 f. Tese (Doutorado) - Instituto de Matemática e Estatística, Universidade de São Paulo, São Paulo, 2012.

Nesta tese, estudamos um arcabouço, introduzido por Frank, que denominamos de sistemas generalizados de núcleos. Provamos teoremas sobre empacotamentos de certos objetos combinatórios neste arcabouço, tanto para o caso inteiro quanto para o fracionário. Estes teoremas, em particular, implicam em uma melhora nos limitantes superiores de Schrijver, para o empacotamento de ramificações, e de Gabow e Manu, para o empacotamento de arborescências. Além disso, também provamos que o problema de minimização num poliedro relacionado pode ser resolvido em tempo polinomial, dado um oráculo de separação.

Palavras-chave: arborescências, ramificações, laminaridade, submodularidade, supermodularidade. 


\section{Abstract}

\section{Rey, M. L. A general framework for packing branchings and other combinatorial structures.}

2012. 91 f. Tese (Doutorado) - Instituto de Matemática e Estatística, Universidade de São Paulo, São Paulo, 2012.

We study a framework, which we call a generalized kernel system, introduced by Frank. We prove some integral and fractional packing theorems on this framework which, in particular, imply an improvement on the best upper bounds currently known, one due to Schrijver, for packing branchings from a given root-sets, and another, due to Gabow and Manu, for packing spanning arborescences from a given root. We also establish the polynomial time complexity, modulo a separation oracle, of a related minimization problem involving a polyhedron derived from this framework.

Keywords: arborescences, branchings, laminarity, submodularity, supermodularity. 


\section{Sumário}

$\begin{array}{ll}\text { Lista de Abreviaturas } & \text { ix }\end{array}$

Lista de Símbolos $\quad$ xi

1 Introdução 1

1.1 O problema do empacotamento . . . . . . . . . . . . . . . . . . 1

1.2 Sistemas de biconjuntos intersectantes . . . . . . . . . . . . . . 3

1.3 Sistemas capacitados e irrestritos de núcleos . . . . . . . . . . . . . . . . 6

1.4 Definições básicas . . . . . . . . . . . . . . . . . . . . . . . . . 9

1.5 Organização . . . . . . . . . . . . . . . . . . . . 10

2 O arcabouço dos sistema generalizados de núcleos 13

2.1 Posets intersectantes . . . . . . . . . . . . . . . . . . . 13

2.2 Sistemas generalizados de núcleos . . . . . . . . . . . . . . . . 15

2.3 Uma generalização das ideias de Lovász e Frank . . . . . . . . . . . . . . . . 18

2.4 Algumas consequências . . . . . . . . . . . . . . . . . . . . 22

3 Sistemas generalizados de núcleos com a propriedade do cruzamento 31

3.1 Sistemas generalizados de núcleos com a propriedade do cruzamento . . . . . . . . 31

3.2 Um procedimento de descruzamento . . . . . . . . . . . . . . . . . . . 32

3.3 Um algoritmo alternativo . . . . . . . . . . . . . . . . . 33

4 Sistemas generalizados de núcleos com uma família mista 37

4.1 Famílias mistas . . . . . . . . . . . . . . . . . . . . 37

4.2 Alguns teoremas sobre empacotamentos . . . . . . . . . . . . . . . 43

5 Empacotamentos em sgnfm $\quad 47$

5.1 Dois teoremas sobre empacotamentos . . . . . . . . . . . . . . 47

5.2 Mais dois teoremas sobre empacotamentos . . . . . . . . . . . . . . . 58

6 Alguns poliedros $\quad \mathbf{6 5}$

6.1 Uma caracterização do politopo das coberturas . . . . . . . . . . . . . . . . . 65

6.2 A integralidade de um certo poliedro supermodular . . . . . . . . . . . . . . . 67 
viii SUMÁRIO

7 Considerações finais

73

Referências Bibliográficas

Índice Remissivo 


\title{
Lista de Abreviaturas
}

\author{
iposet Poset intersectante \\ pim Propriedade da intersecção mista \\ sgn Sistema generalizado de núcleos \\ sgnmf Sistema generalizado de núcleos com uma família mista \\ sgn-c Sistema generalizado de núcleos com a propriedade do cruzamento \\ sgn-c-fm Sistema generalizado de núcleos com a propriedado cruzamento e uma família mista \\ spi Sequência propriamente intersectante
}




\section{Lista de Símbolos}

$\begin{array}{ll}\rho u & \text { Conjunto dos arcos do núcleo } u \\ c(u) & \text { Capacidade de um núcleo } u \\ \lambda \mathbb{K} & \text { Capacidade de um sgn } \mathbb{K} \\ \Lambda \mathbb{K} & \text { Conjunto dos núcleos de um sgn cuja capacidade é } \lambda \mathbb{K} \\ \mu S & \text { Demanda da parte } \bowtie \text {-fechada } S \\ p(u) & \text { Demanda do núcleo } u \\ \Gamma \mathbb{F} & \text { Conjunto dos núcleos } u \text { de } \mathbb{F} \text { para os quais } c(\rho u)=p(u)>0 \\ \Gamma_{0} \mathbb{F} & \text { Conjunto dos núcleos } u \text { de } \mathbb{F} \text { para os quais } c(\rho u)=p(u) \\ U \mathbb{F} & \text { Conjunto dos núcleos } u \text { de } \mathbb{F} \text { tais que } p(u)>0 \\ f^{+}, \text {supp } f & \text { Suporte de uma função } f \\ \chi^{X} & \text { Função característica de um conjunto } X \\ \text { free }_{S} B & \text { Conjunto dos núcleos } u \text { de } S \text { tais que } \rho u=\emptyset \\ \operatorname{cov}_{S} B & \text { Conjunto dos núcleos de } S \text { cobertos por } B\end{array}$




\section{Capítulo 1}

\section{Introdução}

Neste capítulo, vamos definir os problemas que estudaremos nesta tese. Mostraremos também os resultados conhecidos e destacaremos algumas de nossas contribuições. Além disso, o capítulo também tem por objetivo fixar a notação e definir alguns termos básicos que serão usados durante este trabalho. Salientamos, desde já, que para não interromper o fluxo da leitura, a definição de alguns termos é delegada para o final do capítulo.

\subsection{O problema do empacotamento}

Seja $P$ um conjunto finito, cujos elementos denominamos de núcleos, e $E$ um conjunto finito, cujos elementos denominamos de $\operatorname{arcos}$ e para os quais $P \cap E=\emptyset$. Ademais, seja $\rho$ uma função que leva cada elemento de $P$ em um subconjunto de $E$. Vamos denominar a tripla $\mathbb{K}=(P, E, \rho)$ de sistema irrestrito de núcleos. Seja $B$ uma parte de $E$. O conjunto dos núcleos cobertos por $B$, denotado $\operatorname{cov} B$, é o conjunto $\{u \in P \mid \rho(u) \cap B \neq \emptyset\}$. Dizemos $B$ é uma cobertura de $P$ se $\operatorname{cov} B=P$. Considere uma família disjunta $\mathcal{B}$ de coberturas de $P$. É claro que $|\mathcal{B}| \leqslant|\rho(u)|$ para todo $u \in P$, donde

$$
|\mathcal{B}| \leqslant \min \{|\rho(u)| \mid u \in P\} .
$$

É conveniente, então, definir $\lambda \mathbb{K}:=\min \{|\rho(u)| \mid u \in P\}$. Ademais, escrevemos $\varphi \mathbb{K}$ para denotar a cardinalidade de uma família disjunta máxima de coberturas de $P$. Não é difícil exibir um sistema irrestrito de núcleos para o qual $\varphi \mathbb{K}<\lambda \mathbb{K}$ : tome, por exemplo,

$\triangleright \quad\left(\left\{u_{1}, u_{2}, u_{3}\right\},\{a, b, c\},\left\{\left(u_{1},\{a, b\}\right),\left(u_{2},\{a, c\}\right),\left(u_{3},\{b, c\}\right)\right\}\right)$.

Assim, é natural considerar o seguinte problema: encontrar sistemas irrestritos de núcleos $\mathbb{K}$ para os quais $\varphi \mathbb{K}=\lambda \mathbb{K}$. Neste caso, ou seja, quando a desigualdade minimax vale com igualdade, dizemos que $\mathbb{K}$ empacota.

Parte de nosso interesse, neste trabalho, está em produzir algoritmos eficientes para, por exemplo, decidir se um determinado sistema irrestrito de núcleos empacota. Estes algoritmos tipicamente necessitarão de certos oráculos para calcular um determinado tipo de objeto, que deixaremos explícito no momento apropriado. No entanto, é natural agora, sobretudo em virtude dos exemplos 
que seguem, estabelecer em que condições, módulo os tais oráculos, um algoritmo tem consumo de tempo polinomial neste arcabouço. Dizemos que um algoritmo tem complexidade polinomial se seu consumo de tempo é um polinômio no número de arcos, módulo o número de chamadas aos oráculos destacados na descrição do algoritmo. Assim, é claro que qualquer algoritmo que dependa do tamanho do conjunto de núcleos não é polinomial.

Vamos, agora, exibir alguns exemplos de sistemas irrestritos que empacotam. Começaremos pelo clássico Teorema de Menger [Fra11]. Para isso, serão necessários alguns conceitos elementares de Teoria dos Grafos.

Um digrafo é uma tupla $(V, A, \imath)$ em que $V$ e $A$ são conjuntos finitos e disjuntos, e $\imath: A \rightarrow V \times V$. Cada elemento de $V$ é denominado de vértice, e cada elemento de $A$, de arco. É conveniente definir funções ${ }^{-} \imath, \imath^{+}: A \rightarrow V$ de tal forma que $\imath(a)=\left({ }^{-} \imath(a), \imath^{+}(a)\right)$ para cada arco $a \in A$. Para um arco $a$ em $A$, dizemos que ${ }^{-} a:={ }^{-} \imath(a)$ é a cauda de $a$, e $a^{+}:=\imath^{+}(a)$ é a cabeça de $a$. As pontas de um $\operatorname{arco} a$ em $A$ são os componentes do par $\imath(a)$. Por brevidade, escrevemos $(V, A)$ em vez de $(V, A, \imath)$ ficando então implícitas as funções que produzem a cauda e a cabeça de cada arco.

Seja $D=(V, A)$ um digrafo. Para uma parte $X$ de $V$, o conjuntos dos arcos que entram em $X$, é o conjunto

$\triangleright \quad\left\{a \in A \mid a^{+} \in X,-a \notin X\right\}$,

denotado por $\vec{\delta}(X)$. Para um subconjunto $B$ de $A$, escrevemos $\vec{\delta}_{B}(X)$ para denotar $\vec{\delta}(X) \cap B$. Além disso, pomos $\vec{d}(X):=|\vec{\delta}(X)|$ e, analogamente, $\vec{d}_{B}(X):=\left|\vec{\delta}_{B}(X)\right|$. Para um vértice $r$ de $D$, denotamos por $\mathcal{V}_{D}(\bar{r})$, ou simplesmente $\mathcal{V}(\bar{r})$, o conjunto dos subconjuntos não-vazios de $V$ que não contêm $r$. Analogamente, para vértices $s$ e $t$ de $D$, escrevemos $\mathcal{V}(\bar{s}, t)$, para denotar o conjunto dos subconjuntos de $V$ que contêm $t$ e não contêm $s$.

Podemos, agora, retomar a discussão sobre o Teorema de Menger. Seja $D=(V, A)$ um digrafo, e $s, t$ vértices distintos de $D$. Um st-caminho é uma cobertura minimal de $\mathcal{V}(\bar{s}, t)$. O Teorema de Menger afirma que o sistema irrestrito de núcleos $(\mathcal{V}(\vec{s}, t), A, \vec{\delta})$ empacota. É evidente que o Teorema de Menger admite a seguinte reformulação:

Teorema 1.1 (Menger) Seja $D$ um digrafo, $s \neq t$ vértices de $D$ e $k \in \mathbb{N}$. Então existe uma família disjunta $\mathcal{P}$ de st-caminhos tal que $|\mathcal{P}| \geqslant k$ se e só se $\vec{d}(X) \geqslant k$ para cada $X \in \mathcal{V}(\bar{s}, t)$.

O próximo exemplo é um teorema clássico de Edmonds [Edm73] envolvendo arborescências em digrafos. Seja, então, $D$ um digrafo e $r$ um de seus vértices. Uma $r$-arborescência é uma cobertura minimal de $\mathcal{V}(\bar{r})$. A versão fraca do Teorema das Arborescências de Edmonds afirma que o sistema irrestrito de núcleos $(\mathcal{V}(\bar{r}), A, \vec{\delta})$ empacota. A versão fraca do Teorema de Edmonds, similarmente ao Teorema de Menger, admite a seguinte reformulação:

Teorema 1.2 (Edmonds [Edm73]) Seja D um digrafo, $r$ um de seus vértices e $k \in \mathbb{N}$. Então existe uma família disjunta $\mathcal{P}$ de r-arborescências tal que $|\mathcal{P}| \geqslant k$ se e só se $\vec{d}(X) \geqslant k$ para cada $X \in \mathcal{V}(\bar{r})$.

Vamos, agora, exibir um arcabouço, introduzido por Frank [Fra79], que captura, dentre outros objetos combinatórios, tanto o empacotamento de $s t$-caminhos como o de $r$-arborescências. Seja $\mathcal{F}$ uma família de partes de $V$. Dizemos que $\mathcal{F}$ é intersectante se para todo $X, Y \in \mathcal{F}$ : 
$\triangleright \quad X \cap Y \neq \emptyset$ implica que $X \cup Y \in \mathcal{F}$ e $X \cap Y \in \mathcal{F}$.

O par $(D, \mathcal{F})$ é um sistema de núcleos se $\mathcal{F}$ é intersectante. O Teorema 1.3, a seguir, devido a Frank [Fra79], afirma que o sistema irrestrito de núcleos $(\mathcal{F}, A, \vec{\delta})$ empacota. É essencial destacar que a prova de tal fato é uma generalização de uma prova de Lovász [Lov76] para a versão fraca do Teorema de Edmonds. Vale ressaltar também que o argumento usado na prova depende fortemente do fato de que a função $\vec{d}$ é submodular, isto é, para todo $X, Y \subseteq V$ :

$$
\vec{d}(X)+\vec{d}(Y) \geqslant \vec{d}(X \cup Y)+\vec{d}(X \cap Y) .
$$

Teorema 1.3 (Frank [Fra79]) Seja $(D, \mathcal{F})$ um sistema de núcleos e $k \in \mathbb{N}$. Então D possui uma família disjunta $\mathcal{B}$ de coberturas de $\mathcal{F}$ tal que $|\mathcal{B}| \geqslant k$ se e só se $\vec{d}(X) \geqslant k$ para cada $X \in \mathcal{F}$.

Há diversas formas de atacar o problema mencionado no início desta seção. Uma motivação é procurar por arcabouços mais gerais (que os sistemas de núcleos) onde a essência da ideia envolvida na prova de Lovász, da versão fraca do Teorema de Edmonds, pode ainda ser aplicada com sucesso. Uma parte importante desta tarefa foi desenvolvida inicialmente por Frank [Fra79], para os sistemas de núcleos, e depois estendida por Szegő [Sze01], como veremos adiante. Há ainda uma extensão de Bérczi e Frank [BF08] que inclui estes dois últimos arcabouços. Neste trabalho, vamos mostrar que as ideias de Lovász podem ser aplicadas num contexto ainda mais geral. A seguir, para ilustrar, vamos apresentar um teorema - e sua respectiva prova - envolvendo um arcabouço que inclui como casos particulares os Teoremas 1.1, 1.2 e 1.3 e que servirá como um prenúncio da prova de um teorema mais geral que apresentaremos no Capítulo 2.

\subsection{Sistemas de biconjuntos intersectantes}

Vamos, agora, exibir uma prova de uma versão mais fraca de um Teorema de Frank [Fra09], que, em particular, contém como casos especiais os Teoremas 1.1, 1.2 e 1.3. A prova, cuja organização é original, é uma extensão de uma prova de Frank [Fra79] do Teorema 1.3. Enfatizamos, todavia, que a prova serve como um prenúncio do que faremos adiante. Além disso, é importante destacar que os sistemas de biconjuntos intersectantes, definidos no que segue, constituem uma abstração conveniente para lidar com problemas envolvendo vértice-conectividade em digrafos; veja, por exemplo, [Fra99, Fra09]. Antes de apresentarmos este resultado, necessitamos de algumas definições preliminares.

Seja $D=(V, A)$ um digrafo. Um biconjunto é um par $X=\left(X_{I}, X_{O}\right)$ tal que $\triangleright \quad \emptyset \neq X_{I} \subseteq X_{O} \subseteq V$.

Para biconjuntos $X$ e $Y$, escrevemos $X \sqsubseteq Y$ se $X_{I} \subseteq Y_{I}$ e $X_{O} \subseteq Y_{O}$. Para uma família $\mathcal{R}$ de biconjuntos, dizemos que $X \in \mathcal{R}$ é minimal (maximal) em $\mathcal{R}$ se $Y \in \mathcal{R}$ e $Y \sqsubseteq X(X \sqsubseteq Y)$ implicam que $X=Y$. Além disso, definimos

$\triangleright \quad X \sqcup Y:=\left(X_{I} \cup Y_{I}, X_{O} \cup Y_{O}\right) \quad$ e $\quad X \sqcap Y:=\left(X_{I} \cap Y_{I}, X_{O} \cap Y_{O}\right)$. 
É evidente que $X \sqcap Y$ é um biconjunto se, e só se, $X_{I} \cap Y_{I} \neq \emptyset$. Um arco $a$ de $D$ entra em $X$ (ou cobre $X$ ) se $a \in \vec{\delta} X_{I} \cap \vec{\delta} X_{O}$. Uma parte $B$ de $A$ entra em $X$ (ou cobre $X$ ) se existe $a \in B$ tal que $a$ entra em $X$. Vamos sobrecarregar a notação e escrever $\vec{\delta} X$ para denotar o conjunto dos arcos que entram em $X$. Além disso, estendendo-se naturalmente a notação previamente definida pomos $\vec{d}(X):=|\vec{\delta}(X)|$. É fácil estabelecer que para todo par $X, Y$ de biconjuntos:

$$
\vec{d} X+\vec{d} Y \geqslant \vec{d}(X \sqcup Y)+\vec{d}(X \sqcap Y) .
$$

Vamos destacar algumas propriedades que, embora elementares, serão usadas frequentemente na argumentação do teorema principal desta seção.

(i) Para cada tripla $X, Y, Z$ de biconjuntos e cada $a \in A$ :

se $X \sqsubseteq Y \sqsubseteq Z$ e $a$ entra em $X$ e em $Z$, então $a$ entra em $Y$.

(ii) Para cada dupla $X, Y$ de biconjuntos e cada $a \in A$ :

se $a$ entra em $X \sqcup Y$, então $a$ entra em $X$ ou $Y$, e

(iii) Para cada dupla $X, Y$ de biconjuntos e cada $a \in A$ :

se $X_{I} \cap Y_{I} \neq \emptyset$ e $a$ entra em $X \sqcap Y$, então $a$ entra em $X$ ou $Y$, e

Sistemas de biconjuntos intersectantes Uma coleção $\mathcal{F}$ de biconjuntos é intersectante se para todo $X, Y \in \mathcal{F}$ :

$\triangleright \quad X_{I} \cap Y_{I} \neq \emptyset$ implica $X \sqcup Y, X \sqcap Y \in \mathcal{F}$.

Um sistema de biconjuntos intersectantes, ou simplesmente um sbi, é um par $\mathbb{K}=(D, \mathcal{F})$ em que $D=(V, A)$ é um digrafo e $\mathcal{F}$ é uma coleção intersectante de biconjuntos. O grau de $\mathbb{K}$, denotado $\lambda \mathbb{K}$, é o número

$\triangleright \quad \min \{\vec{d} X \mid X \in \mathcal{F}\}$.

Dizemos que $\mathbb{K}$ é não-trivial se $\lambda \mathbb{K}>0$. Seja $B$ uma parte de $A$. A família dos biconjuntos de $\mathcal{F}$ coberta por $B$ é denotada por $\operatorname{cov} B$. Dizemos que $B$ cobre $\mathcal{F}$ se $\operatorname{cov} B=\mathcal{F}$, ou seja, $B$ entra em $X$ para cada $X \in \mathcal{F}$. Também dizemos que $X \in \mathcal{F}$ de $V$ é evitado por $B$ se $B \cap \vec{\delta} X=\emptyset$. Definimos $\mathbb{K}^{B}:=(D-B, \mathcal{F})$.

Arbóreo Este é um conceito fundamental que aparecerá em outras ocasiões. Um subconjunto $B$ de $A$ é arbóreo se para cada $X, Y \in \mathcal{F}$ :

$\triangleright \quad$ se $X \sqsubseteq Y$ e $B$ entra em $X$, então $B$ entra em $Y$.

Lema 1.4 Seja $\mathbb{K}$ um sbi e B uma parte arbórea de A. Se U é um biconjunto maximal em $\mathcal{F}$ evitado por $B$, e $a \in \vec{\delta} U$, então $B \cup\{a\}$ é arbóreo.

Prova. Suponha, por contradição, que exista um biconjunto $U$ maximal que é evitado por $B$, e um arco $a \in \vec{\delta} U$ tal que $B \cup\{a\}$ não é arbóreo. Então existem $X, Y \in \mathcal{F}$ tais que

$$
X \sqsubseteq Y, \quad B \cup\{a\} \text { entra em } X, \quad \text { e } \quad B \cup\{a\} \text { evita } Y .
$$


Como $B$ é arbóreo, então $B$ evita $X$ e $Y$. Assim, $a$ entra em $X$ e evita $Y$. Como $a$ entra em $X$ e $X \sqsubseteq Y$, então $a^{+} \in Y_{I}$. Mas $a$ evita $Y$, donde ${ }^{-} a \in Y_{O}$. Agora, $a$ entra em $U, \operatorname{logo} a^{+} \in U_{I} \mathrm{e}^{-} a \notin U_{O}$. Portanto, $U \sqcup Y \sqsupset U$. No entanto, a maximalidade de $U$ implica que $B$ entra em $U \sqcup Y$, donde, devido a (1.1.ii), $B$ entra em $U$ ou $Y$, o que é uma contradição.

Lema 1.5 Seja $\mathbb{K}=(D, \mathcal{F})$ um sbi não-trivial e $B$ uma parte de $A$.

Se $B$ é arbóreo e $\lambda\left(\mathbb{K}^{B}\right) \geq \lambda \mathbb{K}-1$ e $B$ não cobre $\mathcal{F}$, então existe $a \in A \backslash B$ tal que

(i) $\lambda\left(\mathbb{K}^{B \cup\{a\}}\right) \geqslant \lambda \mathbb{K}-1$,

(ii) $B \cup\{a\}$ é arbóreo, $e$

(iii) $\operatorname{cov}(B \cup\{a\}) \supset \operatorname{cov} B$.

Prova. Suponha que $B$ é arbóreo e não cobre $\mathcal{F}$. Seja $U$ um biconjunto maximal em $\mathcal{F}$ que $B$ evita. É claro que $\vec{\delta} U=\vec{\delta}_{D-B} U$. Seja $\mathcal{R}:=\left\{X \in \mathcal{F} \mid \vec{d}_{D-B}(X)=\lambda \mathbb{K}-1\right\}$. O objetivo agora consiste em mostrar que existe um arco que entra em $U$ mas não entra em nenhum elemento de $\mathcal{R}$.

Fato 1.5.1 A família $\mathcal{R}$ é intersectante.

- Sejam $X, Y \in \mathcal{R}$. Então

$$
\lambda-1+\lambda-1=\vec{d}_{D-B}(X)+\vec{d}_{D-B}(Y) \geqslant \vec{d}_{D-B}(X \sqcup Y)+\vec{d}_{D-B}(X \sqcap Y) \geqslant \lambda-1+\lambda-1,
$$

e, por conseguinte, $\vec{d}_{D-B}(X \sqcup Y)=\vec{d}_{D-B}(X \sqcap Y)=\lambda-1$, donde $X \sqcup Y, X \sqcap Y \in \mathcal{R}$.

Seja $\mathcal{J}:=\left\{X \in \mathcal{R} \mid U_{I} \cap X_{I} \neq \emptyset\right\}$. Suponha, primeiro, que $\mathcal{J}=\emptyset$. Como $\mathbb{K}$ é não-trivial e $B$ evita $U$, então existe $a \in \vec{\delta} U$; ademais, $a$ evita cada biconjunto em $\mathcal{R}$, o que estabelece (i). Mas, o Lema 1.5 implica que $B \cup\{a\}$ é arbóreo, o que prova (ii). Além disso, $B \cup\{a\}$ cobre $U$ e, consequentemente, $\operatorname{cov}(B \cup\{a\}) \supset \operatorname{cov} B$, o que garante (iii).

Suponha, agora, que $\mathcal{J} \neq \emptyset$. Seja $R$ um biconjunto minimal em $\mathcal{J}$.

Fato 1.5.2 Existe $a \in \vec{\delta} U \backslash \vec{\delta} R$ tal que $a \in \vec{\delta}(U \sqcap R)$.

- Como $B$ é arbóreo e $B$ entra em $R$, então $R \nsubseteq U$. Ademais, $B$ arbóreo implica que $B$ evita $U \sqcap R$ e, consequentemente, $\vec{d}_{D-B}(U \sqcap R)=\vec{d}(U \sqcap R) \geqslant \lambda$ o qual, aliado à $\vec{d}_{D-B}(R)=\lambda-1$, implica na existência de $a \in \vec{\delta} U \backslash \vec{\delta} R$ tal que $a \in \vec{\delta}(U \sqcap R)$.

Seja, então, $a$ o arco cuja existência é garantida pelo Fato 1.5.2.

Fato 1.5.3 Para todo $S \in \mathcal{R}: a$ evita $S$.

- Suponha, por contradição, que $a$ entra em $S$ para algum $S \in \mathcal{R}$. Ora, $a$ entra em $U \sqcap R$, donde $a^{+} \in R_{I} \cap S_{I}$. Mas, $a$ evita $R$ e, daí, ${ }^{-} a \in R_{O}$. Ademais, ${ }^{-} a \notin S_{O}$. Logo, $R \sqcap S \sqsubset R$. De $R, S \in \mathcal{R}$, pelo Fato 1.5.1, vem que $R \sqcap S \in \mathcal{R}$. Agora, $a$ entra em $R \sqcap S$. De fato, de ${ }^{-} a \notin S_{O}$, vem que ${ }^{-} a \notin R_{O} \cap S_{O}$ o que, combinado com $a^{+} \in R_{I} \cap S_{I}$, implica que $a$ entra em $R \sqcap S$. Finalmente, de $a$ entra em $U$ e em $R \sqcap S$ vem que $U_{I} \cap(R \sqcap S)_{I} \neq \emptyset$, donde $R \sqcap S \in \mathcal{J}$, o que contraria a minimalidade de $R$. 
O Fato 1.5.3 implica que $\lambda\left(\mathbb{K}^{B \cup\{a\}}\right) \geqslant \lambda \mathbb{K}-1$, o que garante (i). O Lema 1.5 implica que $B \cup\{a\}$ é arbóreo — provando, assim, (ii) — e, finalmente, a escolha de $a$ estabelece (iii), pois $\operatorname{cov}(B \cup\{a\}) \supset \operatorname{cov} B$. Isto completa a prova do lema.

O próximo teorema pode ser obtido usando-se o lema anterior aliado a um argumento indutivo corriqueiro e, por isso, sua prova é omitida.

Teorema 1.6 (Frank [Fra09]) Seja $\mathbb{K}=(D, \mathcal{F})$ um sbi, $e k \in \mathbb{N}$.

Então existe uma família disjunta $\mathcal{B}$ de coberturas de $\mathcal{F}$ tal que $|\mathcal{B}| \geqslant k$ se e só se $\lambda \mathbb{K} \geqslant k$.

\subsection{Sistemas capacitados e irrestritos de núcleos}

Um sistema capacitado e irrestrito de núcleos é uma tupla $\mathbb{K}=(P, E, \rho, c)$, em que $(P, E, \rho)$ é um sistema irrestrito de núcleos e $c: E \rightarrow \mathbb{R}_{+}$. Quando a imagem de $c$ está em $\mathbb{N}$ dizemos que o sistema é inteiro. Estendemos, de forma natural, a noção de grau através da definição:

$\triangleright \quad \lambda \mathbb{K}:=\min \{c(u) \mid u \in P\}$,

onde, por simplicidade, escrevemos $c(u):=c(\rho(u)):=\sum[c(a) \mid a \in \rho(u)]$ para cada $u \in P$.

Seja, agora, $\mathcal{B}$ a família das coberturas de $P$. Dizemos que $y: \mathcal{B} \rightarrow \mathbb{R}_{+}$é viável se

$\triangleright \quad \sum\left[y(B) \chi^{B} \mid B \in \mathcal{B}\right] \leqslant c$.

Além disso, seja $\varphi \mathbb{K}:=\max \left\{\sum[y(B) \mid B \in \mathcal{B}] \mid y\right.$ é viável $\}$ e considere o problema de decidir se $\mathbb{K}$ empacota, ou seja, se $\varphi \mathbb{K}=\lambda \mathbb{K}$. Se um tal $y$, que atinge o máximo existe, então dizemos que y é um empacotamento de $\mathbb{K}$. Note que se $\mathbb{K}$ é inteiro e $(P, E, \rho)$ empacota, então $\mathbb{K}$ também empacota. É claro que isto pode ser provado através da adição de $c(a)$ cópias de cada $\operatorname{arco} a \in E$. No entanto, o empacotamento produzido, digamos $y$, é tal que seu suporte, $y^{+}$, tem tamanho $\lambda \mathbb{K}$, o que evidentemente não é, em geral, um polinômio em $|E|$. Assim, uma pergunta natural consiste em decidir se existem empacotamentos cujo suporte é um polinômio no número de arcos.

Considere uma generalização natural da versão fraca do Teorema de Edmonds que envolve adicionar capacidades, isto é, números não-negativos aos arcos do digrafo. Seja, então, $D=(V, A)$ um digrafo e $c: A \rightarrow \mathbb{R}_{+}$uma função capacidade. Seja também $r$ um vértice de $D$. Edmonds [Edm73] estabeleceu que o sistema capacitado e irrestrito de núcleos $\mathbb{D}:=(\mathcal{V}(\bar{r}), A, \vec{\delta}, c)$ empacota. Gabow e Manu [GM98] provaram o seguinte teorema que garante a existência de um empacotamento com poucas coberturas.

Teorema 1.7 (Gabow e Manu [GM98]) Seja $D=(V, A)$ um digrafo, $c: A \rightarrow \mathbb{R}_{+}$uma função capacidade, e r um vértice de $D$. Então $(\mathcal{V}(\bar{r}), A, \vec{\delta}, c)$ possui um empacotamento y tal que

(i) $\left|y^{+}\right| \leqslant|A|$.

(ii) se c é inteiro, então yé inteiro e $\left|y^{+}\right| \leqslant|A|+|V|-2$

Vamos mostrar, no Capítulo 5, que é possível obter um empacotamento cujo suporte é não mais que $|A|-|V|+2$ e, no caso inteiro, não mais que $|A|$. 
É conveniente, agora, discutir brevemente e de forma superficial, um algoritmo de Gabow e Manu [GM98] para o empacotamento fracionário de arborescências geradoras, uma vez que tal algoritmo será generalizado neste trabalho. Ressaltamos que este algoritmo foi estendido por Barahona [Bar04] para o problema do empacotamento fracionário de $T$-junções e, por Matsuoka [Mat12], para o problema do empacotamento fracionário em clutters ideais.

O algoritmo de Gabow e Manu [GM98], para o empacotamento fracionário de arborescências, produz um empacotamento cujo suporte é não superior ao número de arcos do digrafo. Para uma parte $B$ de $A$ e um real positivo $\varepsilon$, seja $c^{\varepsilon B}:=c[B \ni a \mapsto c(a)-\varepsilon]$. O sistema capacitado e irrestrito de núcleos, obtido de $\mathbb{D}$, pela remoção de $\varepsilon$ cópias de $B$, isto é, $\left(\mathcal{V}(\bar{r}), A, \vec{\delta}, c^{\varepsilon B}\right)$ é denotado por $\mathbb{D}^{\varepsilon B}$. A ideia central do algoritmo consiste em selecionar uma $r$-arborescência $B \subseteq c^{+} \mathrm{e}$ remover um número máximo, digamos $\varepsilon$, de cópias de $B$ de tal forma que $\lambda\left(\mathbb{D}^{\varepsilon B}\right) \geqslant \lambda \mathbb{D}-\varepsilon$. Surpreendentemente esta estratégia produz em empacotamento cujo suporte é não superior ao número de $\operatorname{arcos}$ de $D$. A análise que garante este limitante depende de um certo subconjunto laminar de $\{X \in \mathcal{V}(\bar{r}) \mid c(\vec{\delta} X)=\lambda\}$. A cada iteração ou um arco é "zerado", ou esta família laminar aumenta de tamanho.

Gabow e Manu [GM98] também desenvolveram um algoritmo para a versão inteira do problema, produzindo, assim, um empacotamento inteiro. A ideia é similar a do algoritmo para empacotamento fracionário e consiste em selecionar uma $r$-arborescência $B$ e remover um número inteiro máximo, digamos $k$, de cópias de $B$. Se este número $k$ é igual a $\varepsilon$, definido no parágrafo anterior, então empacotam-se estas $k$ cópias e inicia-se uma nova iteração. Se $k<\varepsilon$ então o algoritmo seleciona uma parte maximal $J$ de $B$ tal que $\lambda\left(\left(\mathbb{D}^{B}\right)^{J}\right)=\lambda\left(\left(\mathbb{D}^{B}\right)^{J}\right)-1$ e estende $J$ para uma $r$-arborescência $J^{\prime}$ de $D$. Tal estratégia produz um empacotamento cujo suporte tem tamanho não superior a $|A|+|V|-2$.

\section{Empacotamentos simultâneos}

Vamos, agora, generalizar os modelos vistos anteriormente e considerar o problema do empacotamento simultâneo. Informalmente, são dados subconjuntos de núcleos e suas respectivas demandas. O objetivo é o de encontrar, para cada um de tais subconjuntos, uma família de coberturas e suas respectivas multiplicidades, de tal forma que a demanda de cada conjunto seja satisfeita exatamente e, ademais, nenhum arco seja usado mais vezes que sua capacidade. No que segue, vamos formalizar tais ideias. Suponha que $\mathbb{K}=(P, E, \rho, c)$ é um sistema capacitado e irrestrito de núcleos. Seja $\mu: 2^{P} \rightarrow \mathbb{R}_{+}$uma função demanda e para cada $S \in 2^{P}$, seja $\mathcal{B}_{S}$ a família das coberturas de $S$. Para cada $S \in 2^{P}$, seja $y_{S}: \mathcal{B}_{S} \rightarrow \mathbb{R}_{+}$. Finalmente, seja $y$ a função que leva cada $S \in 2^{P}$ em $y_{S}$. Dizemos que y é um empacotamento de $(\mathbb{K}, \mu)$ se

(i) para cada $S \in 2^{P}: \sum\left[y_{S}(B) \mid B \in \mathcal{B}_{S}\right]=\mu S$, e

(ii) $\sum\left[y_{S}(B) \chi^{B} \mid S \in 2^{P} ; B \in \mathcal{B}_{S}\right] \leqslant c$.

Vamos tecer alguns comentários antes de continuar. É claro que nossa definição de função demanda, que envolve todos os subconjuntos de $P$, é mais uma conveniência que uma necessidade, 
afinal, como na descrição informal, basta fornecer o suporte de $\mu$. Ademais, agora, um algoritmo tem complexidade polinomial se seu consumo de tempo é polinomial no número de arcos e no tamanho do suporte da função demanda (módulo oráculos). Este modelo captura, por exemplo, a versão forte, enunciada abaixo, do Teorema das Arborescências (ou das Ramificações) de Edmonds [Edm73]. Seja $D=(V, A)$ um digrafo. Para uma parte $R$ de $V$, seja $\mathcal{V}(\bar{R}):=\{X \subseteq V \mid$ $X \cap R=\emptyset ; X \neq \emptyset\}$. Uma $R$-ramificação é uma cobertura minimal de $\mathcal{V}(\bar{R})$.

Teorema 1.8 (Edmonds [Edm73]) Seja D um digrafo e $R_{1}, \ldots, R_{k}$ subconjuntos não-vazios de $V$. Então $D$ possui subconjuntos $B_{1}, \ldots, B_{k}$ de $E$, dois a dois disjuntos, tais que $B_{i}$ é uma $R_{i}$-ramificação para cada $i \in[k]$ se e só se $\vec{d}(X) \geqslant\left|\left\{i \in[k] \mid R_{i} \cap X=\emptyset\right\}\right|$ para cada $\emptyset \neq X \subseteq V$.

É evidente que o Teorema 1.8 implica o Teorema 1.2. No entanto, não se conhece uma redução do Teorema 1.2 para o Teorema 1.8. A seguinte generalização, que passamos a descrever, foi estabelecida por Schrijver [Sch03].

Teorema 1.9 (Schrijver [Sch03]) Seja $D=(V, A)$ um digrafo, $c: A \rightarrow \mathbb{N}$ uma função capacidade e $\mu: 2^{V} \rightarrow \mathbb{N}$ uma função demanda $e \mathbb{D}:=\left(2^{V}, A, \vec{\delta}, c\right)$. Para cada $U \subseteq V$, seja

$\triangleright \quad p(U):=\sum\left[\mu(R) \mid R \in 2^{V}, R \cap U=\emptyset\right]$.

Se c $(\vec{\delta}(U)) \geq p(U)$ para cada $U \subseteq V$, então $(\mathbb{D}, \mu)$ possui um empacotamento inteiro y tal que

$$
\sum\left[\left|y_{S}^{+}\right| \mid S \in 2^{V}\right] \leq|A|+\left|\mu^{+}\right|+|V|^{3} .
$$

Vamos, no Capítulo 5, provar um resultado geral a respeito de empacotamentos em um arcabouço que denominamos de sistemas generalizados de núcleos que, em particular, implica na existência de um empacotamento cujo suporte é não superior a $|A|+\left|\mu^{+}\right|+|V|-2$.

$\mathrm{O}$ arcabouço dos sistema de núcleos não contém (ao menos) diretamente a versão forte do Teorema das Arborescências de Edmonds. Para finalizar esta seção, vamos apresentar um arcabouço, devido a Szegó [Sze01], que contorna esta possível limitação. Frisamos, no entanto, que os sistemas generalizados de núcleos, que passaremos a estudar no próximo capítulo, são uma generalização deste e, também, de um outro arcabouço mais geral proposto por Frank e Bércsi [BF08].

Seja $D$ um digrafo e $\mathcal{F}_{1}, \ldots, \mathcal{F}_{k}$ famílias intersectantes, não necessariamente distintas, de subconjuntos de $V$. Dizemos que $\mathcal{F}_{1}, \ldots, \mathcal{F}_{k}$ satisfaz a propriedade da intersecção mista se para todo $i, j \in[k]$ e todo $X \in \mathcal{F}_{i}$ e $Y \in \mathcal{F}_{j}$ :

$\triangleright \quad X \cap Y \neq \emptyset$ implica $X \cap Y \in \mathcal{F}_{i} \cap \mathcal{F}_{j}$.

Para cada subconjunto $X$ de $V$, seja $p(X):=\left|\left\{i \in[k] \mid X \in \mathcal{F}_{i}\right\}\right|$. É claro que se para cada $i \in[k]$ existe uma cobertura $B_{i}$ de $\mathcal{F}_{i}$ e, ademais, $B_{i} \cap B_{j}=\emptyset$ para todo $i \neq j \in[k]$, então $\vec{d}(X) \geqslant p(X)$ para toda parte $X$ de $V$. Szegő [Sze01] estabeleceu que esta última condição além de necessária é também suficiente.

Teorema 1.10 (Szegó [Sze01]) Seja D um digrafo, $k \in \mathbb{N} e \mathcal{F}_{1}, \ldots, \mathcal{F}_{k}$ famílias intersectantes, não necessariamente distintas, de subconjuntos de V. Existe para cada $i \in[k]$ uma cobertura $B_{i}$ de $\mathcal{F}_{i}$ tal que $B_{i} \cap B_{j}=\emptyset$ para todo $i \neq j \in[k]$ se e só se $\vec{d}(X) \geqslant p(X)$ para toda parte $X$ de $V$. 


\subsection{Definições básicas}

Para não tornar o texto repetitivo, vamos desde já estabelecer uma convenção a respeito da notação que vamos usar. Vamos lidar com diversos objetos, tais como digrafos. Para não sobrecarregar a notação vamos tipicamente omitir, quando o contexto permitir, o objeto sobre o qual uma certa função está definida. Assim, por exemplo, o conjunto dos arcos que entram em um certo subconjunto $X$ dos vértices de um digrafo $D$ é denotado por $\vec{\delta}(X)$ em vez de $\vec{\delta}_{D}(X)$ ou, ainda, $\vec{\delta}(X ; D)$. Enfatizamos que tal convenção só será usada quando não houver possibilidade de confusão.

\section{Conjuntos}

Sejam $X$ e $Y$ conjuntos. Então $X$ é uma parte, ou um subconjunto de $Y$ se $x \in X$ implica $x \in Y$; o conjunto $X$ é uma parte própria de $Y$, denotado $X \subset Y$ se $X$ é uma parte de $Y$ e $Y$ não é uma parte de $X$. A diferença dos conjuntos $X$ e $Y$, denotada por $X \backslash Y$, é o conjunto $\{x \in X \mid x \notin Y\}$. Se $X$ é parte de $Y$ então $\bar{X}$, o complemento de $X$ em $Y$, denota o conjunto $Y \backslash X$; é evidente que tal notação só será usada quando o conjunto em relação ao qual tomamos o complemento estiver óbvio do contexto.

Vamos também, às vezes, confundir conjuntos unitários com os seus elementos. Por exemplo, vamos definir alguns conceitos envolvendo conjuntos e para simplificar, quando o conjunto for unitário, isto é, da forma $\{s\}$, vamos escrever $s$ em vez de $\{s\}$.

Uma família é um conjunto cujos elementos também são conjuntos. Assim, uma família é apenas um outro nome para um conjunto. Dizemos que uma família $\mathcal{F}$ é disjunta se

$\triangleright \quad$ para todo $X, Y \in \mathcal{F}: X \neq Y$ implica $X \cap Y=\emptyset$.

O conjunto dos naturais $\{0,1,2, \ldots\}$ é denotado por $\mathbb{N}$; o conjunto dos reais é denotado por $\mathbb{R}$ enquanto que o conjunto dos reais não-negativos, isto é, $\{r \in \mathbb{R} \mid r \geqslant 0\}$ é denotado por $\mathbb{R}_{+}$. Para cada $k \in \mathbb{N}$, adotamos a abreviatura $[k]:=\{1,2, \ldots, k\}$. Desta forma, $[0]=\emptyset$.

\section{Funções}

Sejam $X$ e $Y$ conjuntos. Um subconjunto $f$ de $X \times Y$ é uma função de $X$ em $Y$, o que é denotado por $f: X \rightarrow Y$, se

$\triangleright$ para cada $x \in X$ existe $y \in Y$ tal que $(x, y) \in f$, e

$\triangleright$ para cada $x \in X$ se $(x, y) \in f$ e $\left(x, y^{\prime}\right) \in f$ então $y=y^{\prime}$.

Como de hábito, para cada $x \in X$, o único $y$ tal que $(x, y) \in f$ é denotado por $f(x)$, ou simplesmente $f x$, quando não houver possibilidade de confusão.

Seja $U$ um conjunto, $f: X \rightarrow Y$ e $h: U \rightarrow Y$. Escrevemos $f[U \ni u \mapsto h(u)]$ para denotar a função que coincide com $f$ nos elementos de $X \backslash U$ e coincide com $h$ nos elementos de $U$. Formalmente,

$\triangleright \quad f[U \ni u \mapsto h(u)]:=(f \backslash\{(u, f(u)) \mid u \in U \cap X\}) \cup\{(u, h(u)) \mid u \in U\}$.

Observe que $f[U \ni u \mapsto h(u)]:(X \cup U) \times Y$. Seja $V$ um conjunto e $h: V \rightarrow Y$. Então 
$\triangleright \quad f[U \ni u \mapsto h(u), V \ni v \mapsto g(v)]$

denota a função $(f[U \ni u \mapsto h(u)])[V \ni v \mapsto g(v)]$. Como de costume, quando $U^{\prime}=\{x\}$ para algum $x \in X$, por brevidade, escrevemos $f[x \mapsto h(x)]$. Para cada $y$ em $Y$, escrevemos $\vec{y}_{X}$, ou simplesmente $\vec{y}$, para denotar a função $\emptyset[X \ni x \mapsto y]$.

Para uma função $f$ de $X$ em $\mathbb{R}$, o suporte de $f$, denotado $f^{+}$, ou ainda supp $f$, é o conjunto $\{x \in X \mid f(x)>0\}$.

Finalmente, para um subconjunto $X$ de um conjunto $U$, denotamos por $\chi^{X}$, a função característica de $X$, onde para cada $u \in U$ :

$\triangleright \quad \chi^{X}(u):=$ se $u \in X$ então 1 , senão 0.

\section{Sequências}

Por vezes, vamos ter que lidar com sequências definidas sobre um conjunto. Nossas sequências serão finitas e por isso omitiremos tal adjetivo. Seja, então, $X$ um conjunto e $\mathbf{f}$ uma sequência sobre $X$, isto é, $\mathbf{f}:[k] \rightarrow X$ para algum $k \in \mathbb{N}$. Evidentemente, vamos adotar a convenção habitual e escrever $\left(\mathbf{f}_{1}, \ldots, \mathbf{f}_{k}\right)$ para denotar a sequência $\mathbf{f}$. No entanto, é conveniente também empregar a notação que segue. A sequência vazia, de forma não convencional, será denotada por $\emptyset$, uma vez que os candidatos naturais, $\varepsilon$ e $\lambda$, serão usados com outras finalidades. Se $\mathbf{f}$ é uma sequência finita sobre $X$ e $x$ é um elemento de $X$ então $\mathbf{f} \cdot x$ é a sequência obtida de $\mathbf{f}$ ao adicionarmos $x$ como um último elemento. Formalmente, $\mathbf{f} \cdot x:=\mathbf{f}[(k+1) \mapsto x]$. Definimos também

$\triangleright$ para toda sequência f sobre $X: \mathbf{f} \cdot \emptyset:=\mathbf{f}$, e

$\triangleright$ para todo par $\mathbf{f}, \mathbf{g}$ de sequências sobre $X$ e todo $x \in X: \mathbf{f} \cdot(\mathbf{g} \cdot x):=(\mathbf{f} \cdot \mathbf{g}) \cdot x$.

É claro que $(\mathbf{f} \cdot \mathbf{g}) \cdot \mathbf{h}=\mathbf{f} \cdot(\mathbf{g} \cdot \mathbf{h})$ para quaisquer sequências $\mathbf{f}, \mathbf{g}$ e $\mathbf{h}$ sobre $X$. Para simplificar, às vezes, vamos omitir o operador · e escrever $\mathbf{f g}$ em vez de $\mathbf{f} \cdot \mathbf{g}$. Sejam $\mathbf{f}$ e $\mathbf{g}$ sequências sobre $X$. Dizemos que gé prefixo de $\mathbf{f}$ se existe uma sequência $\mathbf{h}$ tal que $\mathbf{f}=\mathbf{g h}$. É conveniente confundir $\mathbf{f}$ com o conjunto $\{\mathbf{f}(i) \mid i \in[k]\}$. Assim, vamos, por exemplo, escrever $\mathbf{f} \subseteq Y$, onde $Y$ é uma parte de $X$, se a imagem de $\mathbf{f}$ é um subconjunto de $Y$. Ademais, $a \in \mathbf{f}$ é, então, uma abreviatura para existe $i \in[k]$ tal que $a=\mathbf{f}(i)$.

\subsection{Organização}

No Capítulo 2, vamos apresentar os sistemas generalizados de núcleos, que constituem o objeto combinatório sobre o qual dedicaremos grande parte deste trabalho. Neste capítulo estenderemos algumas ideias de Lovász e Frank, cujas origens remontam à prova da versão fraca do Teorema de Edmonds, e provaremos alguns teoremas envolvendo tal arcabouço. No Capítulo 3, vamos exibir um arcabouço, que é uma restrição de um sistema generalizado de núcleos que permite uma certa operação de descruzamento. Com isso, poderemos lidar com a noção de conjuntos laminares e definir operações que permitem obter extensões de conjuntos laminares, usando esta operação de descruzamento. Esta operação permitirá deduzir um algoritmo alternativo para o problema do 
empacotamento neste arcabouço. No Capítulo 4, vamos introduzir os sistemas generalizados de núcleos com uma família mista (sgnfm), que generalizam os sistemas de biconjuntos com a propriedade da intersecção mista. Este últimos, por sua vez, generalizam o problema do empacotamento de ramificações em digrafos. Provaremos diversas propriedades fundamentais dos sgnfms e, em particular, mostraremos um primeiro teorema envolvendo empacotamentos em tais arcabouços. No Capítulo 5, vamos apresentar diversos teoremas sobre empacotamentos em sgnfms e, em sua versão mais restrita, aquela que possui a propriedade do cruzamento. Estes teoremas fornecerão melhores limitantes para o tamanho de um empacotamento, quando especializados para o problema do empacotamento de ramificações e de arborescências em digrafos. Finalmente, no Capítulo 6 vamos provar uma caracterização para o politopo das coberturas de um sistema generalizado de núcleos e, além disso, vamos mostrar que é possível resolver, em tempo polinomial, módulo um oráculo de separação, o problema de minimizar uma função linear no poliedro de um sistema generalizado de núcleos com a propriedade do cruzamento delimitado por uma função supermodular e positiva em seus membros intersectantes. O material contido nestes capítulos, a menos que dito o contrário, é original. 
12 INTRODUÇÃO 


\section{Capítulo 2}

\section{$O$ arcabouço dos sistema generalizados de núcleos}

Neste capítulo, vamos apresentar um arcabouço, devido a Frank [Fra99], que generaliza os diversos arcabouços apresentados na introdução. O principal resultado deste capítulo está contido na Proposição 2.6. Esta proposição generaliza algumas ideias de Lovász [Lov76], estendidas posteriormente, na ordem, por Frank [Fra79], Szegő [Sze01], e, finalmente, por Bérczi e Frank [BF08], a qual permitirá mostrar que tal arcabouço empacota. Além disso, tal proposição contém os ingredientes necessários para estabelecer, como veremos no Capítulo 4, que é possível empacotar simultaneamente, diante de certas condições, uma família de conjuntos, num sentido ainda mais geral, que remete à versão forte do Teorema de Edmonds.

\subsection{Posets intersectantes}

Um poset é um par $(P, \preccurlyeq)$ em que $P$ é um conjunto finito e $\preccurlyeq$ é uma relação binária sobre $P$ tal que para todo $u, v, w \in P$ :

$\triangleright u \preccurlyeq u$,

$\triangleright u \preccurlyeq w$ e $w \preccurlyeq u$ implicam $u=w, \mathrm{e}$

$\triangleright u \preccurlyeq v$ e $v \preccurlyeq w$ implicam $u \preccurlyeq w$.

Tipicamente vamos denominar cada elemento de $P$ de núcleo. Para $u, v \in P$, escrevemos $u \prec v$, se $u \preccurlyeq v$ e $u \neq v$. Para uma parte $X$ de $P$, um núcleo $u \in X$ é minimal em $X$ (maximal em $X$ ) se não existe $t \in X$ tal que $t \prec u(u \prec t)$. Para um subconjunto $X$ de $P$, o conjunto dos elementos minimais (maximais) de $X$ é denotado por $\check{X}(\widehat{X})$. Para cada $v \in P$, seja

$\triangleright \quad v^{\downarrow}:=\{u \in P \mid u \preccurlyeq v\}$.

Dizemos que $u, v \in P$ são comparáveis, denotado $u \sim v$, se $u \preccurlyeq v$ ou $v \preccurlyeq u$. Também dizemos que $u, v \in P$ são propriamente intersectantes, denotado $u \bowtie v$, se

$\triangleright \quad u \nsim v \quad$ e $\quad u^{\downarrow} \cap v^{\downarrow} \neq \emptyset$. 
É conveniente, para simplificar, escrever $u \widetilde{\bowtie} v$ se $u \bowtie v$ ou $u \sim v$. Um subconjunto $L$ de $P$ é laminar se nenhum par de seus elementos é propriamente intersectante, isto é,

$\triangleright \quad$ para cada $\ell, m \in L: \ell \not \Delta m$.

iposet Um poset $P$ é intersectante, ou simplesmente um iposet, se $P$ é munido de duas operações comutativas $\vee$ e $\wedge$, definidas sobre cada par $(u, w) \in P \times P$ tal que $u \widetilde{\bowtie} w$, e que satisfazem para cada $u, w \in P$ :

(o.1) se $u \preccurlyeq w$, então $u \wedge w=u$ e $u \vee w=w$,

(o.2) se $u \bowtie w$, então $u \wedge w \prec u$ e $u \wedge w \prec w$, e $u \prec u \vee w$ e $w \prec u \vee w$.

Há duas noções de conjuntos fechados que vamos usar. Seja, então, $X$ uma parte de $P$. Dizemos que $X$ é $\bowtie$-fechada, se para cada $u, w \in X$ :

$\triangleright \quad u \bowtie w$ implica $u \wedge w \in X$ e $u \vee w \in X$.

Por fim, dizemos que $X$ é $\wedge$-fechada, se para cada $u, w \in X$ :

$\triangleright \quad u \bowtie w$ implica $u \wedge w \in X$.

Para simplificar, vamos sobrecarregar a notação, e quando o contexto permitir, vamos escrever $P$ no lugar do mais longo $(P, \preccurlyeq, \vee, \wedge)$.

O próximo lema será útil num vindouro procedimento de descruzamento. Vamos, no entanto, exibí-lo agora em virtude seu de caráter básico e por depender tão somente dos axiomas que acabamos de enumerar. Vamos, entretanto, necessitar da seguinte definição que será usada frequentemente nesta tese. Para uma parte laminar $L$ de $P$, e $u \in P$, denotamos por $L_{u}$, o conjunto dos núcleos maximais $\ell \in L$ tais que $\ell \bowtie u$, ou seja, $\ell \in L_{u}$ se e só se

$\ell \bowtie u \quad$ e $\quad(m \bowtie u$ e $m \sim \ell$ implicam $m \preccurlyeq \ell)$.

Lema 2.1 Para cada iposet $P$, cada subconjunto $\wedge$-fechado I de P, cada subconjunto laminar $L$ de $I$, e cada subconjunto $X$ de $I$, se $X \neq \emptyset$ então existe $u \in X$ tal que

para cada $\ell \in L_{u}$ e cada $z \in I: z \prec \ell$ implica $z \notin X$.

Prova. Suponha, por contradição, que existe um iposet $P$, um subconjunto $\wedge$-fechado $I$ de $P$, uma parte laminar $L$ de $I$, e um subconjunto não-vazio $X$ de $I$ tais que

para cada $u \in X$ existem $\ell \in L_{u}$ e $z \in X$ tais que $z \prec \ell$,

Para cada $u \in X$, fixe arbitrariamente $\ell_{u} \in L_{u}$ e $z_{u} \in X$ tais que $z_{u} \prec \ell_{u}$. Selecione $u \in X$ para o qual $\ell_{u}$ é minimal, isto é, não existe $v \in X$ tal que $\ell_{v} \prec \ell_{u}$. Como $z_{u} \in X$, então a laminaridade de $L$ e a minimalidade de $\ell_{u}$ implicam

$$
\ell_{u} \preccurlyeq \ell_{z_{u}}, \quad \text { ou } \quad\left(\ell_{z_{u}} \nsim \ell_{u} \quad \text { e } \quad \ell_{z_{u}}^{\downarrow} \cap \ell_{u}^{\downarrow}=\emptyset\right)
$$

Lembre-se que, por definição, $\ell_{z_{u}} \bowtie z_{u}$. Admita, primeiro, que $\ell_{u} \preccurlyeq \ell_{z_{u}}$. Neste caso, $z_{u} \prec \ell_{u} \preccurlyeq \ell_{z_{u}}$ contradiz $\ell_{z_{u}} \bowtie z_{u}$. Suponha, agora, que $\ell_{z_{u}} \nsim \ell_{u}$ e $\ell_{z_{u}}^{\downarrow} \cap \ell_{u}^{\downarrow}=\emptyset$. Como $\ell_{z_{u}} \bowtie z_{u}$ e $I$ é $\wedge$-fechado, 
então $\ell_{z_{u}} \wedge z_{u}$ está em $I$. Mas $\ell_{z_{u}} \wedge z_{u} \prec \ell_{z_{u}}$ e, ademais, $\ell_{z_{u}} \wedge z_{u} \prec z_{u} \prec \ell_{u}$. Portanto, $\ell_{z_{u}} \wedge z_{u} \in \ell_{z_{u}}^{\downarrow} \cap \ell_{u}^{\downarrow}$, o que é uma contradição.

É claro que a prova do lema anterior sugere o seguinte algoritmo que recebe um iposet $P$, uma parte laminar $L$ de $I$, e um subconjunto não-vazio $X$ de $I$, onde $I$ é uma parte $\bowtie$-fechada de $P$, e devolve um núcleo $u \in X$ tal que

para cada $\ell \in L_{u}$ e cada $z \in I: z \prec \ell$ implica $z \notin X$.

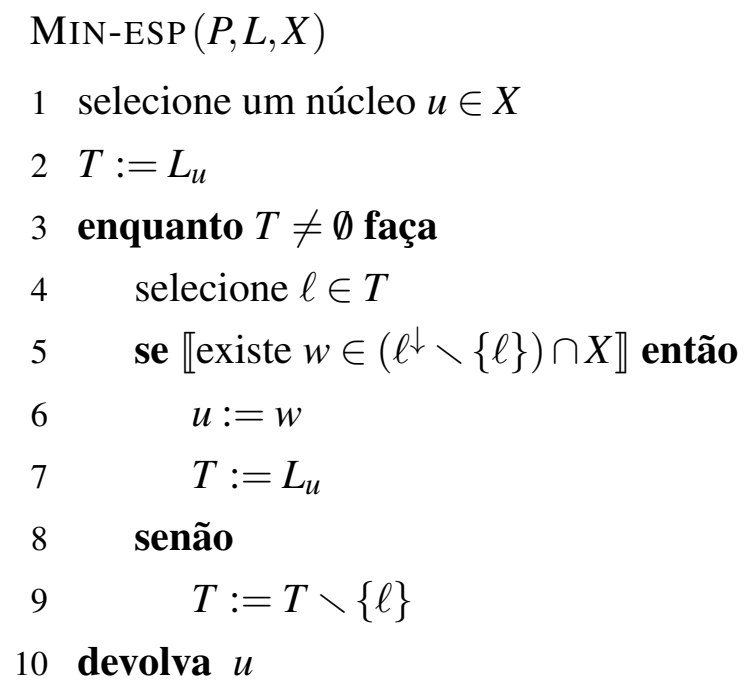

É oportuno, agora, tecer um primeiro comentário sobre a complexidade computacional dos algoritmos. Nesta tese, questões de complexidade computacional, embora fundamentais, não serão discutidas com profundidade. Nossa maior preocupação está em estabelecer polinomialidade em função de certos oráculos, os quais, nos algoritmos que vamos descrever, serão exibidos

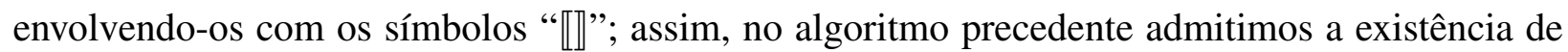
um oráculo que determina, se existe, um $w \in\left(\ell^{\downarrow} \backslash\{\ell\}\right) \cap X$. Em relação ao algoritmo acima ainda dispomos de poucos elementos para determinar quais parâmetros serão considerados para estabelecer sua complexidade. É claro que o número de iterações do algoritmo está limitado por $|L|$. Além disso, se supusermos que $P$ é parte da entrada e que o oráculo tem complexidade de tempo polinomial, então o algoritmo também tem consumo de tempo polinomial. No entanto, para as aplicações que veremos, $P$ tipicamente terá tamanho exponencial em outro parâmetro de entrada.

\subsection{Sistemas generalizados de núcleos}

Seja $P$ um iposet, $E$ um conjunto finito, disjunto de $P$, cujos elementos são denominados de

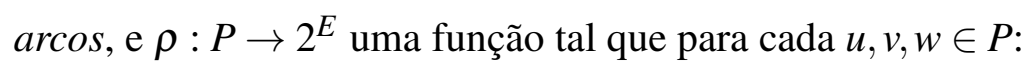

( $\rho .1)$ se $u \preccurlyeq v \preccurlyeq w$, então $\rho u \cap \rho w \subseteq \rho v$,

$(\rho .2)$ se $u \bowtie v$, então $\rho(u \vee v) \cup \rho(u \wedge v) \subseteq \rho u \cup \rho v$, e

( $\rho .3)$ se $\rho u \cap \rho v \neq \emptyset$, então $u \widetilde{\bowtie} v$. 
Lembre-se que $\rho u$ é uma notação alternativa para $\rho(u)$. Seja $B$ uma parte de $E$. Escrevemos $\triangleright \quad \rho_{B}(u):=(\rho u) \cap B \quad$ e $\quad d_{B}(u):=\left|\rho_{B}(u)\right|$

para cada $u \in P$. Por brevidade, escrevemos $d(u)$ em vez de $d_{E}(u)$ para cada $u \in P$. Também definimos para cada $u \in P$ :

$\triangleright \quad c(u):=c(\rho u):=\sum[c(a) \mid a \in \rho u]$,

e esta notação é transportada para outros contextos onde uma função é definida sobre elementos de algum conjunto, e aplicamos a função a um subconjunto de elementos.

Um sistema generalizado de núcleos, ou simplesmente um sgn é uma tupla

$\triangleright \quad(P, \preccurlyeq, \vee, \wedge, E, \rho, c)$,

onde $(P, \preccurlyeq, \vee, \wedge)$ é um iposet, $\rho$ satisfaz $(\rho .0)-(\rho .3)$ e $c: E \rightarrow \mathbb{R}_{+}$é uma função capacidade. Quando $c: P \rightarrow \mathbb{N}$, dizemos que o sgn é inteiro. Isto completa a definição do arcabouço introduzido por Frank [Fra99]. É conveniente também definir

$$
\begin{aligned}
& \triangleright \lambda \mathbb{K}:=\min \{c(u) \mid u \in P\}, \mathrm{e} \\
& \triangleright \Lambda \mathbb{K}:=\{u \in P \mid c(u)=\lambda \mathbb{K}\} .
\end{aligned}
$$

Dizemos que $\mathbb{K}$ é não-trivial se $\lambda \mathbb{K}>0$. Note, entretanto, que $\lambda \mathbb{K}=0$ e $\rho u \neq \emptyset$ para todo $u \in S$ podem, evidentemente, ser válidos simultaneamente. Quando o contexto permitir, vamos, como de hábito, escrever $\lambda$ e $\Lambda$ em vez de $\lambda \mathbb{K}$ e $\Lambda \mathbb{K}$.

Vamos, tipicamente, denotar por $\mathbb{K}$ um sgn arbitrário e, além disso, devido à nossa convenção sobre denotação de iposets, supomos $\mathbb{K}=(P, \rho, c)$, deixando implícito o conjunto dos arcos - o qual, salvo o dito o contrário, será denotado por $E$ - sobre o qual tanto $\rho$ quanto $c$ são definidas. Quando nenhuma função capacidade é dada, admitimos $c=d$ e, neste caso, dizemos que $\mathbb{K}$ é $n a \tilde{o}$ capacitado. Vamos evitar, sempre que possível, o subscrito $\mathbb{K}$ nas funções que dependem de $\mathbb{K}$. Para as funções $\rho$ e $d$ e também para as operações $\vee, \wedge$, o contexto deixará claro a qual sgn estas funções se referem, donde nenhuma de tais referências será necessária.

O próximo lema estabelece a submodularidade de certas funções que serão fundamentais para os sistemas generalizados de núcleos.

\section{Lema 2.2 Seja $\mathbb{K} u m \operatorname{sgn} e u, w \in P \operatorname{com} u \bowtie w$. Então}

(i) para cada $a \in E: d_{\{a\}}(u)+d_{\{a\}}(w) \geqslant d_{\{a\}}(u \vee w)+d_{\{a\}}(u \wedge w)$,

(ii) para cada $B \subseteq E: d_{B}(u)+d_{B}(w) \geqslant d_{B}(u \vee w)+d_{B}(u \wedge w)$, $e$

(iii) $c(u)+c(w) \geqslant c(u \vee w)+c(u \wedge w)$.

Prova. Sejam $u, w \in P$ tais que $u \bowtie w$. Para a prova de (i), seja $a \in E$. Se $a \in \rho(u \wedge w) \backslash \rho(u \vee w)$ ou $a \in \rho(u \vee w) \backslash \rho(u \wedge w)$ então, por $(\rho .2), a \in \rho u \cup \rho w$ e, portanto,

$$
d_{\{a\}}(u)+d_{\{a\}}(w) \geqslant d_{\{a\}}(u \vee w)+d_{\{a\}}(u \wedge w) .
$$

Suponha, agora, que $a \in \rho(u \wedge w) \cap \rho(u \vee w)$. De $u \wedge w \prec u, w \prec u \vee w$ vem, usando $(\rho .1)$, que $a \in \rho u$ e $a \in \rho w$, donde 


$$
d_{\{a\}}(u)+d_{\{a\}}(w)=d_{\{a\}}(u \vee w)+d_{\{a\}}(u \wedge w) .
$$

o que estabelece (i).

Para a prova (ii), seja $B \subseteq E$. Então

$$
\begin{aligned}
& d_{B}(u)+d_{B}(w)=\sum_{a \in B} d_{\{a\}}(u)+\sum_{a \in B} d_{\{a\}}(w) \\
& =\sum_{a \in B}\left(d_{\{a\}}(u)+d_{\{a\}}(w)\right) \geqslant \sum_{a \in B}\left(d_{\{a\}}(u \vee w)+d_{\{a\}}(u \wedge w)\right) \\
& =\sum_{a \in B} d_{\{a\}}(u \vee w)+\sum_{a \in B} d_{\{a\}}(u \wedge w)=d_{B}(u \vee w)+d_{B}(u \wedge w),
\end{aligned}
$$

onde a primeira desigualdade segue em virtude de (i).

Para a prova de (iii), observe que

$$
\begin{aligned}
& c(u)+c(w)=\sum_{a \in E} c(a) d_{\{a\}}(u)+\sum_{a \in E} c(a) d_{\{a\}}(w) \\
& =\sum_{a \in E} c(a)\left(d_{\{a\}}(u)+d_{\{a\}}(w)\right) \geqslant \sum_{a \in E} c(a)\left(d_{\{a\}}(u \vee w)+d_{\{a\}}(u \wedge w)\right) \\
& =\sum_{a \in E} c(a) d_{\{a\}}(u \vee w)+\sum_{a \in E} c(a) d_{\{a\}}(u \wedge w)=c(u \vee w)+c(u \wedge w),
\end{aligned}
$$

onde a primeira desigualdade vem de $c \geqslant 0$ e de (i). Isto completa a prova do lema.

\section{Lema 2.3 Seja $\mathbb{K}$ um sgn, e u,w núcleos em P tais que}

$$
u \bowtie w \quad e \quad c(u)+c(w)=c(u \vee w)+c(u \wedge w) .
$$

\section{Então}

(i) se $a \in c^{+}$, então $d_{\{a\}}(u)+d_{\{a\}}(w)=d_{\{a\}}(u \vee w)+d_{\{a\}}(u \wedge w)$.

(ii) se $\rho(u) \cup \rho(w) \subseteq c^{+}$, então $\chi^{\rho u}+\chi^{\rho w}=\chi^{\rho(u \vee w)}+\chi^{\rho(u \wedge w)}$.

Prova. É claro que $\chi^{\rho v}(a)=d_{\{a\}}(v)$ para todo $v \in P$ e todo $a \in E$. Observe, agora, que

$$
\begin{aligned}
& c(u)+c(w) \\
& =\sum_{a \in E} d_{\{a\}}(u) c(a)+\sum_{a \in E} d_{\{a\}}(w) c(a) \\
& =\sum_{a \in E} c(a)\left(d_{\{a\}}(u)+d_{\{a\}}(w)\right) \\
& \geqslant \sum_{a \in E} c(a)\left(d_{\{a\}}(u \vee w)+d_{\{a\}}(u \wedge w)\right) \\
& =c(u \vee w)+c(u \wedge w) \\
& =c(u)+c(w),
\end{aligned}
$$

onde a desigualdade segue do Lema 2.2. Assim, vale a igualdade em todas as passagens e, portanto,

$$
\sum_{a \in E} c(a)\left(d_{\{a\}}(u)+d_{\{a\}}(w)\right)=\sum_{a \in E} c(a)\left(d_{\{a\}}(u \vee w)+d_{\{a\}}(u \wedge w)\right) .
$$

Seja $a \in c^{+}$. Então, em virtude de $(*)$ e do Lema $2.2 \mathrm{i}$, temos que

$$
d_{\{a\}}(u)+d_{\{a\}}(w)=d_{\{a\}}(u \vee w)+d_{\{a\}}(u \wedge w),
$$


o que estabelece (i). Para a prova de (ii), suponha que $\rho u \cup \rho w \subseteq c^{+}$. Seja $a \in E$. Se $a \in c^{+}$então, devido a $(i)$, temos que

$$
\chi^{\rho u}(a)+\chi^{\rho w}(a)=\chi^{\rho(u \vee w)}(a)+\chi^{\rho(u \wedge w)}(a) .
$$

Se $a \notin c^{+}$então $a \in E \backslash(\rho u \cup \rho w)$, donde, em virtude de $(\rho .2), a \notin \rho(u \vee w) \cup \rho(u \wedge w)$ e daí

$$
\chi^{\rho u}(a)+\chi^{\rho w}(a)=\chi^{\rho(u \vee w)}(a)+\chi^{\rho(u \wedge w)}(a),
$$

o que completa a prova de (ii).

\subsection{Uma generalização das ideias de Lovász e Frank}

Nesta seção, vamos provar que uma generalização de um algoritmo de Frank [Fra79], o qual é inspirado em algoritmo de Lovász [Lov76], pode ser usado para estabelecer que sistemas generalizados de núcleos empacotam. A generalização que vamos apresentar é suficiente também, como veremos no Capítulo 4, para deduzir, em particular, um teorema, similar em espírito à versão forte do Teorema de Edmonds, que mostrará que é possível empacotar simultaneamente coberturas de uma família de subconjuntos de núcleos. Antes, entretanto, vamos precisar de algumas definições.

Seja $\mathbb{K}$ um sgn, $B$ uma parte de $c^{+}$e $u$ um núcleo em $P$. Dizemos que $B$ cobre $u$ se $\rho_{B}(u) \neq \emptyset$, e para $X \subseteq P$, dizemos que $B$ cobre $X$, ou é uma cobertura de $X$, se $B$ cobre cada $u \in X$. Vale repetir que uma cobertura, por definição, contém somente elementos do suporte de $c$. Para um subconjunto $S$ de $P$, escrevemos $\operatorname{cov}_{S} B$ para denotar o conjunto

$\triangleright \quad\{u \in S \mid B$ cobre $u\}$.

Por outro lado, dizemos que $B$ evita $u$ se $\rho_{B}(u)=\emptyset$, e $B$ evita uma parte $R$ de $P$ se $B$ evita cada um dos núcleos em $R$; finalmente, para um subconjunto $S$ de $P$, o conjunto

$\triangleright \quad\{u \in S \mid B$ evita $u\}$

é denotado por free ${ }_{S} B$. Para simplificar, quando $S=P$, escrevemos free $B$ e $\operatorname{cov} B$ no lugar de free ${ }_{P} B$ e $\operatorname{cov}_{P} B$, respectivamente.

\section{Conjuntos arbóreos}

Os conjuntos arbóreos nos sgns constituem uma generalização dos conjuntos de arcos de um digrafo que contêm ramificações. Eis sua definição formal. Seja $\mathbb{K}$ um sgn e $S$ uma parte de $P$. Um subconjunto $B$ de $E$ é $S$-arbóreo se

$\triangleright \quad$ para cada $u, v \in S$ : se $u \prec v$ e $B$ cobre $u$, então $B$ cobre $v$.

Quando $S=P$, para abreviar, dizemos que $B$ é arbóreo. Além disso, para uma parte $X$ de $P$ dizemos que $B$ é boa para $X$ se

$\triangleright \quad$ para cada $x \in X: d_{B}(x) \leqslant 1$. 
Lema 2.4 Seja $\mathbb{K}$ um sgn, e S uma parte $\bowtie$-fechada de P. Para cada $B \subseteq c^{+}$, cada u maximal em free $_{S} B$, e cada $e \in \rho u \cap c^{+}$,

(i) se Bé S-arbóreo, então $B \cup\{e\}$ é $S$-arbóreo, e

(ii) se B é S-arbóreo e boa para $\check{S}$, então $B \cup\{e\}$ é $S$-arbóreo e boa para $\check{S}$.

Prova. Para a prova de (i), admita que $B$ é uma parte $S$-arbórea de $c^{+}$e suponha, por contradição, que existem $u$ maximal em free $_{S} B$ e $e \in \rho u \cap c^{+}$tais que $B^{\prime}:=B \cup\{e\}$ não é $S$-arbóreo. Então existem $\ell, m \in S$ tais que

$$
\ell \prec m, \quad B^{\prime} \text { evita } m, \quad \text { e } \quad B^{\prime} \text { cobre } \ell .
$$

Como $B^{\prime}$ evita $m$ e $B \subset B^{\prime}$, então $B$ também evita $m$. Note que $u \neq m$, pois $e \in \rho u \backslash \rho m$. Ora, $B$ é $S$-arbóreo, donde $B$ evita $\ell$. No entanto, $B^{\prime}$ cobre $\ell$ e, daí, $e$ cobre $\ell$. De $e \in \rho u \cap \rho \ell$, usando ( $\left.\rho .3\right)$, vem que $u \widetilde{\bowtie} \ell$.

Fato 2.4.1 $\ell \prec u$.

- Suponha, por contradição, que $u \bowtie \ell$. Lembre-se que $B$ evita $u$ e $\ell$ o qual, combinado com ( $\rho .2)$, implica que $B$ evita $u \vee \ell$. Note que $u \vee \ell \in S$, pois $S$ é $\bowtie$-fechado. Agora, devido a (o.2), $u \prec u \vee \ell$, o que é uma contradição com a maximalidade de $u$ em free $B$. Assim, $u \sim \ell$ o que, aliado a $\ell \in$ free $_{S} B$ e $u$ maximal em free $B$, acarreta $\ell \preccurlyeq u$; além disso, $\ell \prec u$ pois, caso contrário, $u=\ell \prec m$ e $m \in$ free $_{S} B$ contraria a maximalidade de $u$ em free $B$.

Temos, assim, que $\ell \prec u$ e $\ell \prec m$. Se $u \neq m$, então $u \bowtie m$. De $B$ evita $u$ e $m$, combinado com ( $\rho .2)$, segue que $B$ evita $u \vee m$. No entanto, $u \vee m \in S$, donde $u \vee m \in$ free $_{S} B$ o que, mais uma vez, contraria a maximalidade de $u$ em free $B$. Logo, $u \sim m$. Finalmente, a maximalidade de $u$ e $m \neq u$ implicam que $m \prec u$ e, consequentemente, $\ell \prec m \prec u$. Agora, $(\rho .1)$ e $e \in \rho \ell \cap \rho u$ acarretam $e \in \rho m$, uma contradição. Portanto, $B^{\prime}$ é $S$-arbóreo, como queríamos.

Para a prova de (ii), suponha que $B$ é $S$-arbóreo e bom para $\breve{S}$. O conjunto $B^{\prime}$ é $S$-arbóreo, em virtude de (i). Falta verificar que $B^{\prime}$ é bom para $\check{S}$. Seja $v \in \check{S}$. Então $d_{B}(v) \leqslant 1$. Suponha, por contradição, que $d_{B^{\prime}}(v) \geqslant 2$. Neste caso, $e \in \rho u \cap \rho v$, donde $u \widetilde{\bowtie} v$; ademais, $d_{B}(v)=1$ o que, aliado à $d_{B}(v)=0$, acarreta $u \neq v$. Ora, $u \bowtie v$ uma vez que $v \in \check{S}$ e $S$ é $\bowtie$-fechado e, consequentemente, $u \sim v$. A minimalidade de $v$ em $S$ implica que $v \prec u$. Entretanto, $d_{B}(v)=1$ e $u \in$ free $_{S} B$ contradizem o caráter $S$-arbóreo de $B$. Concluímos, assim, que $B$ é bom para $\breve{S}$, o que completa a prova de (ii) e do lema.

Corolário 2.5 Seja $\mathbb{K}$ um sgn não-trivial, e $S$ uma parte $\bowtie$-fechada de P. Então $\mathbb{K}$ possui uma cobertura de $S$ boa para $\check{S}$.

Prova. Seja $B$ uma parte maximal de $c^{+}$que é $S$-arbórea e boa para $\breve{S}$. Suponha que $B$ não cobre $S$. Tome $u$ maximal em free $B$. Como $\mathbb{K}$ é não trivial, então $\rho u \cap c^{+} \neq \emptyset$. Seja, então, $e \in \rho u \cap c^{+}$. Agora, o Lema 2.4 implica que $B^{\prime}:=B \cup\{e\}$ é uma parte de $c^{+}$que é $S$-arbórea e boa para $\check{S}$, o que contraria a maximalidade de $B$.

É claro que a prova do Corolário 2.5 sugere o seguinte algoritmo que recebe um sgn não-trivial $\mathbb{K}$ e uma parte $\bowtie$-fechada $S$ de $P$ e devolve uma cobertura de $S$, boa para $\check{S}$. 
Algoritmo Cobertura-BoA $(\mathbb{K}, S)$

$1 \quad B:=\emptyset$

2 enquanto free $B \neq \emptyset$ faça

3 【selecione $u$ maximal em free $B \rrbracket$

$4 \quad$ selecione $a \in \rho u \cap c^{+}$

$5 \quad B:=B \cup\{a\}$

6 devolva $B$

Retomando-se a discussão do início do capítulo e relembrando-se os comentários do Capítulo 1, podemos agora mencionar com mais precisão alguns aspectos de complexidade computacional. Os sgns são arcabouços bem gerais e para a maior parte de suas aplicações o conjunto dos núcleos será normalmente muito grande. Para as aplicações envolvendo digrafos tal conjunto será tipicamente exponencial no número de vértices. Assim, vamos dizer que um algoritmo tem complexidade polinomial se seu consumo de tempo é um polinômio no número de arcos do sgn, módulo os oráculos utilizados pelo algoritmo. Lembre-se que vamos identificar nos algoritmos uma chamada a um oráculo colocando-a delimitada pelos símbolos “包”. Desta forma, no algoritmo COBERTURA-BOA a operação "selecione $u$ maximal em free ${ }_{S} B$ " constitui uma chamada para o oráculo. Esta convenção será usada no decorrer de todo este texto e, por isso, não mais a repetiremos.

Como de hábito, admitindo-se que o oráculo acima possui complexidade polinomial, é claro que o algoritmo também possui complexidade polinomial uma vez que o número de iterações está limitado pelo número de arcos do sgn.

\section{A generalização}

No Capítulo 1, exibimos, implicitamente, um algoritmo que produz um empacotamento de um sistema de bi-conjuntos intersectantes. Vamos fazer uma breve digressão a respeito de tal algoritmo. Suponha, assim, que $(D, \mathcal{F})$ é um sbi. A ideia central da prova, da qual é possível derivar um algoritmo, era manter uma parte arbórea $B$ de $A$ tal que $\lambda\left(\mathbb{K}^{B}\right) \geqslant \lambda \mathbb{K}-1$ e mostrar que era possível estender $B$ através da adição de um arco $a$ tal que $B \cup\{a\}$ é arbóreo e, ademais, não entra em nenhum bi-conjunto da família a ser evitada, a saber, $\left\{X \in \mathcal{F} \mid \vec{d}_{D-B}(X)=\lambda \mathbb{K}-1\right\}$. Vamos mostrar que podemos estender estas ideias para o arcabouço dos sgns. Já vimos no Lema 2.4 que conjuntos arbóreos podem ser estendidos de forma a produzir novos conjuntos arbóreos. Além disso, vamos identificar algumas propriedades do conjunto a ser evitado. A identificação destas propriedades permitirá derivar diversas versões e estratégias para a determinação de coberturas em sgns.

Seja $\mathbb{K}$ um sgn. Para uma parte $B$ de $E$ e um real positivo $\varepsilon$ seja

$$
\triangleright \quad c^{\varepsilon B}:=c[B \ni a \mapsto c(a)-\varepsilon]=c-\varepsilon \chi^{B} .
$$

Denotamos por $\mathbb{K}^{\varepsilon B}$ a tripla $\left(P, \rho, c^{\varepsilon B}\right)$ a qual, evidentemente, é um sgn se e só se $c^{\varepsilon B} \geqslant 0$. Lembre- 
se que um subconjunto $R$ de $P$ é $\wedge$-fechado se para todo $r, s \in R$ :

$\triangleright \quad r \bowtie s$ implica $r \wedge s \in R$.

Seja $B$ uma parte de $c^{+}, S$ uma parte $\bowtie$-fechada de $P, R$ uma parte de $P$ e $\varepsilon$ um número real positivo. Dizemos que $(R, S, B, \varepsilon)$ é evitável se

(n.0) $r \in R, s \in S$ e $r \widetilde{\bowtie} s$ implicam $r \wedge s \in S$,

(n.1) $R$ é $\wedge$-fechado,

(n.2) $R \cap$ free $_{S} B=\emptyset$,

(n.3) para cada $r \in R, s \in S \operatorname{com} r \widetilde{凶} s:$

free $_{S} B \ni r \wedge s \prec r$ implica $c^{\varepsilon B}(r \wedge s)>c^{\varepsilon B}(r)$, e

(n.4) para cada $r, s \in R$ :

$r \bowtie s$ implica $c^{\varepsilon B}(r)+c^{\varepsilon B}(s)=c^{\varepsilon B}(r \vee s)+c^{\varepsilon B}(r \wedge s)$.

Proposição 2.6 Seja $\mathbb{K}$ um sgn não-trivial, $R$ uma parte de $P$, $S$ uma parte $\bowtie$-fechada de $P, B$ uma parte $S$-arbórea de $c^{+}$, e $\varepsilon$ um real positivo. $S e(R, S, B, \varepsilon)$ é evitável e $B$ não cobre $S$, então existe $a \in c^{+}$tal que

(i) a evita $R$,

(ii) $B \cup\{a\}$ é S-arbóreo,

(iii) $\operatorname{cov}_{S}(B \cup\{a\}) \supset \operatorname{cov}_{S} B$,

(iv) se $B$ é bom para $\breve{S}$, então $B \cup\{a\}$ também é bom para $\breve{S}$.

Prova. Suponha que $B$ não cobre $S$, e seja $u$ um núcleo maximal em free $B$.

Fato 2.6.1 Existe $a \in \rho u \cap c^{+}$tal que $a$ evita $R$.

- Seja $I:=\{t \in R \mid t \widetilde{\bowtie} u\}$. Suponha, primeiro, que $I=\emptyset$. Como $B$ evita $u$ e $\mathbb{K}$ é não-trivial, então $c^{\varepsilon B}(u)=c(u)>0$ e, por conseguinte, existe $a \in \rho u \operatorname{com} c(a)>0$. Seja $t \in R$. De $I=\emptyset$, aliado à ( $\rho .3)$, vem que $\rho t \cap \rho u=\emptyset \mathrm{e}$, portanto, $a$ evita $R$, o que estabelece o Fato 2.6.1, neste caso.

Suponha, no que segue, que $I$ é não-vazio e seja $r$ um núcleo minimal em $I$, isto é, $r \in \check{I}$. Observe que $u \wedge r \in S$, em virtude de (n.0).

Fato 2.6.1.1 $u \wedge r \prec r \quad$ e existe $a \in\left(c^{+} \cap \rho u \cap \rho(u \wedge r)\right) \backslash \rho r$.

•• Note que $u \wedge r \in$ free $_{S} B$. De fato, como $u \wedge r \preccurlyeq u$ então $u \wedge r=u$ ou $u \wedge r \prec u$. No primeiro caso, não há mais nada a provar. Suponha, pois, que $u \wedge r \prec u$. Neste caso, como $B$ é $S$-arbóreo e $u \in$ free $_{S} B$, então $u \wedge r \in$ free $_{S} B$, como queríamos. De $r \in R$ e $u \wedge r \in$ free $_{S} B$ segue, em virtude de (n.2), que $u \wedge r \prec r$. Assim, usando-se $(n .3)$ obtemos que $c^{\varepsilon B}(u \wedge r)>c^{\varepsilon B}(r)$ e, portanto, existe $a \in \rho(u \wedge r) \backslash \rho r \operatorname{com} c(a)>0$. Note que $(\rho .2)$ implica que $a \in \rho u$, o que completa a prova do Fato 2.6.1.1.

Seja, então, $a$ o arco cuja existência é garantida pelo fato acima.

Fato 2.6.1.2 Para cada $s \in R$ : se $a \in \rho s$ então $s \in I$ e $r \bowtie s$. 
- Seja $s \in R$ e suponha que $a \in \rho s$. Então $r \neq s$ pois $a \notin \rho r$. Note, entretanto, que $a \in \rho s \cap \rho u$ e daí, em virtude de $(\rho .3)$, segue que $s \widetilde{\bowtie} u$, donde $s \in I$.

Resta provar que $r \bowtie s$. Vamos primeiro estabelecer que $r \not s$. Suponha, por contradição, que $r \sim s$. Como $r \in \check{I}, s \in I$ e $r \neq s$, então $r \prec s$. No entanto, $u \wedge r \prec r \prec s, a \in \rho(u \wedge r) \cap \rho s$, e ( $\rho .1)$ implicam $a \in \rho r$, uma contradição. Assim, $r \neq s$. De $a \in \rho(u \wedge r) \cap \rho s$ segue, por ( $\rho .3)$, que $(u \wedge r) \widetilde{\bowtie} s$. Suponha que $(u \wedge r) \sim s$. Se $u \wedge r=s$, então $s=u \wedge r \preccurlyeq r$ contraria $r \neq s$. Se $u \wedge r \prec s$, então de $u \wedge r \prec r$ segue que $r \bowtie s$. Se $s \prec u \wedge r$, então $s \prec u \wedge r \prec r$ implica $s \prec r$, novamente uma contradição com $r \not s$. Admita, finalmente, que $(u \wedge r) \bowtie s$. Então $(u \wedge r) \wedge s$ está em $S$ e de $(u \wedge r) \wedge s \prec u \wedge r \prec r$ e $(u \wedge r) \wedge s \prec s$ vem que $r \bowtie s$. Isto completa a prova do Fato 2.6.1.2.

Se $a \notin \rho s$ para todo $s \in R$ então não há mais nada a provar, pois $a \notin B$. Suponha, então, que $a \in \rho s$ para algum $s \in R$. O Fato 2.6.1.2 implica que $r \bowtie s$. No entanto, como $r, s \in R$ e $R$ é $\wedge$ fechado então $r \wedge s \in R$. Vamos, agora, provar que $a \in \rho(r \wedge s)$. Como $c^{\varepsilon B}(a)=c(a)>0$, então (n.4) e o Lema $2.3 \mathrm{i}$ acarretam

$$
d_{\{a\}}(r)+d_{\{a\}}(s)=d_{\{a\}}(r \wedge s)+d_{\{a\}}(r \vee s),
$$

o que, por sua vez, combinado com $a \notin \rho r$ e $a \in \rho s$ implicam que $a \in \rho(r \wedge s)$ ou $a \in \rho(r \vee s)$. Suponha que $a \in \rho(r \vee s)$. Então $a \in \rho(u \wedge r) \cap \rho(r \vee s)$. Mas, $u \wedge r \prec r \prec r \vee s$, e $(\rho .1)$ implicam que $a \in \rho r$, o que é uma contradição. Logo, $a \in \rho(r \wedge s)$. No entanto, como $a \in \rho(r \wedge s) \cap \rho u$, então $(r \wedge s) \widetilde{\bowtie} u$. Assim, $r \wedge s \in I$. Mas, de $r \bowtie s$ vem que $r \wedge s \prec r$. Daí $I \ni r \wedge s \prec r$ contraria a minimalidade de $r$. Concluímos, assim, que $a$ evita $R$, completando a prova do Fato 2.6.1.

O Fato 2.6.1 estabelece (2.1i). Pelo Lema 2.4, $B \cup\{a\}$ é $S$-arbóreo, donde vale (2.1ii). Ademais, $B \cup\{a\}$ cobre $u$ e $B$ evita $u$ estabelece (2.1iii). Finalmente, (2.1iv) também segue em virtude do Lema 2.4 .

Desta forma, a Proposição 2.6 garante a existência de uma extensão de um conjunto arbóreo diante de certas restrições. A próxima subseção se dedicará a exibir algumas de suas consequências.

\subsection{Algumas consequências}

Vamos agora examinar algumas consequências e aplicações da Proposição 2.6. Nos próximos capítulos vamos investigar algumas aplicações um pouco mais sofisticadas.

A definição seguinte será útil na derivação de consequências da Proposição 2.6. Seja $\mathbb{K}$ um sgn, $B$ um subconjunto de $E$ e $\varepsilon$ um número real. Note que $\mathbb{K}^{\varepsilon B}$ é um sgn se e só se $c^{\varepsilon B} \geqslant 0$. Dizemos que o par $(B, \varepsilon)$ é positivo se

$\triangleright \quad 0<\varepsilon \leqslant \min \{c(e) \mid e \in B\} \quad$ e $\quad \lambda\left(\mathbb{K}^{\varepsilon B}\right) \geqslant \lambda \mathbb{K}-\varepsilon$.

É evidente que se $(B, \varepsilon)$ é positivo então $B \subseteq c^{+}$.

Lema 2.7 Seja $\mathbb{K}$ um $\mathbf{s g n}$ inteiro, $B \subseteq E$ tal que $(B, 1)$ é positivo, e $R:=\left\{u \in P \mid c^{B}(\rho u)=\lambda-1\right\}$. Então 
(i) $(R, P, B, 1)$ é evitável, $e$

(ii) se a $\in c^{+}$evita $R$, então $(B \cup\{a\}, 1)$ também é positivo.

Prova. Vamos primeiro provar que $R$ é $\bowtie$-fechado. Sejam $u, w \in R$ tais que $u \bowtie w$. Então

$$
\begin{aligned}
& \lambda-1+\lambda-1=c^{B}(u)+c^{B}(w) \\
& \geqslant c^{B}(u \vee w)+c^{B}(u \wedge w) \geqslant \lambda-1+\lambda-1,
\end{aligned}
$$

onde a primeira desigualdade é válida pelo Lema $2.2 \mathrm{e}$, a segunda, porque $(B, 1)$ é positivo. Assim, vale a igualdade em todas as passagens, donde $u \vee w, u \wedge w \in R$.

A propriedade (n.0) é válida, uma vez que $P$ é $\bowtie$-fechado; $(n .1)$ é válida, pois $R$ é $\bowtie$-fechado; (n.2) é trivial; (n.3) é válida, uma vez que $c^{B}(u)>c^{B}(r)$ para todo $u \in$ free $B, r \in R$; finalmente, (n.4) é válida, pois $c^{B}(u)=c^{B}(w)$ para todo $u, w \in R$. Isto estabelece (i). Para ver (ii), admita que $a \in c^{+}$evita $R$. Se $a$ cobre $u \in P$, então $c$ inteiro implica $c^{B}(u) \geqslant \lambda$ e, daí, $c^{B \cup\{a\}}(u) \geqslant \lambda-1$. Portanto, $\lambda\left(\mathbb{K}^{B \cup\{a\}}\right) \geqslant \lambda \mathbb{K}-1$, como queríamos.

Teorema 2.8 Seja $\mathbb{K}$ um sgn inteiro e não-trivial.

Então $\mathbb{K}$ possui uma cobertura $B$ de P boa para $\check{P}$ tal que $\lambda\left(\mathbb{K}^{B}\right)=\lambda \mathbb{K}-1$.

Prova. Seja $B$ uma parte maximal de $c^{+}$que é arbórea, boa para $\check{P}$ e tal que $(B, 1)$ é positivo. Suponha, por contradição, que $B$ não é uma cobertura de $P$. Seja $R:=\left\{u \in P \mid c^{B}(u)=\lambda-1\right\}$. O Lema 2.7 implica que $(R, P, B, 1)$ é evitável e, assim, pela Proposição 2.6, existe um arco $a \in c^{+}$ que evita $R$ e para o qual $B \cup\{a\}$ é arbóreo, bom para $\check{P}$ e $\operatorname{cov}(B \cup\{a\}) \supset \operatorname{cov} B$. No entanto, o Lema 2.7 implica que $(B \cup\{a\}, 1)$ é positivo, o que é contrário a maximalidade de $B$. Isto estabelece $\operatorname{cov} B=P$, como queríamos.

Note que o teorema admite a seguinte reformulação.

Teorema 2.9 Seja $\mathbb{K}$ um sgn inteiro e não-trivial. Seja B uma parte arbórea de E, boa para $\check{P}$, tal que $(B, 1)$ é positivo. Então existe uma cobertura $B^{\prime}$ de $P$, boa para $\check{P}$, e tal que

$$
B^{\prime} \supseteq B \quad e \quad \lambda \mathbb{K}^{B^{\prime}}=\lambda \mathbb{K}-1 . \quad \diamond
$$

Usando o Teorema 2.8, uma simples indução em $\lambda \mathbb{K}$ estabelece o resultado seguinte.

Teorema 2.10 Seja $\mathbb{K}$ um sgn não-capacitado.

Então o número máximo de coberturas disjuntas de P que são boas para $\check{P}$ é igual a $\lambda \mathbb{K}$. $\diamond$

Ou ainda, mais geralmente:

Teorema 2.11 Todo sgn inteiro empacota. $\diamond$

É claro que a discussão precedente sugere o seguinte algoritmo que recebe um sgn inteiro $\mathbb{K}$ e devolve um empacotamento de $\mathbb{K}$. 


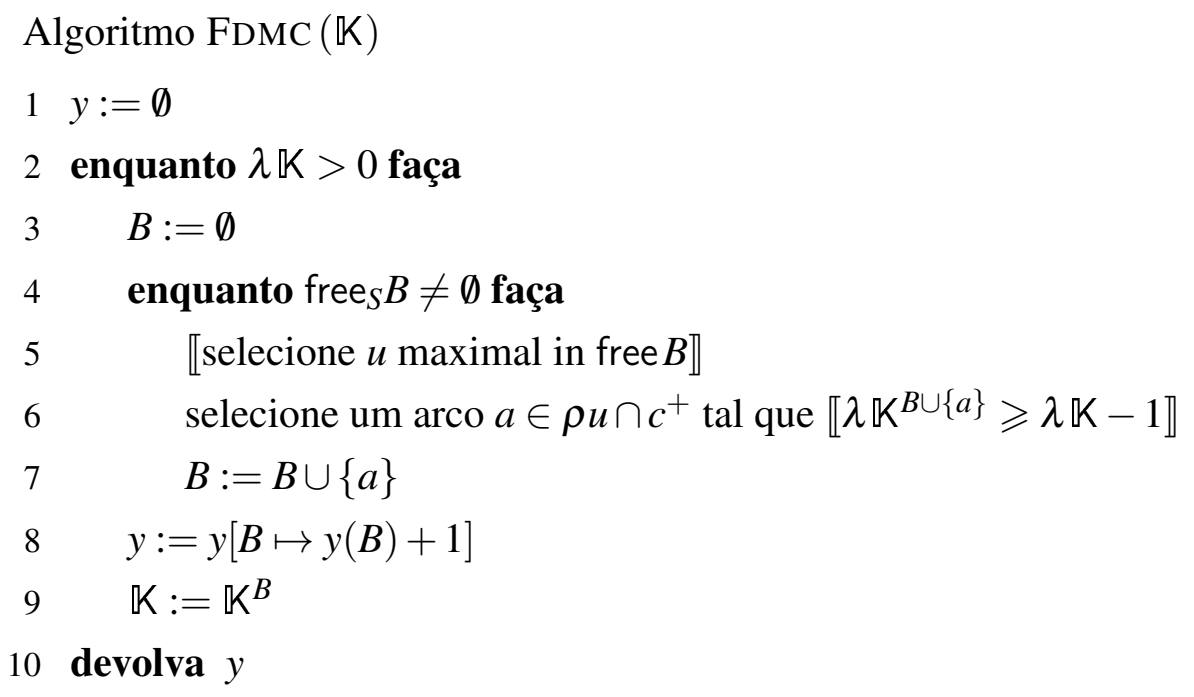

É claro que este algoritmo tem complexidade polinomial se $\mathbb{K}$ é não-capacitado. Ademais, neste caso, $y^{+}$é uma coleção disjunta de coberturas de $P$, boas para $\check{P}$. Observe, entretanto, que, em geral, o algoritmo não é polinomial.

O Teorema 2.11 generaliza, dentre outros, os Teoremas 1.1, 1.2, 1.3 e 1.6 tanto em suas versões capacitadas (inteiras) quanto, evidentemente, as não-capacitadas.

\section{Uma prova e um algoritmo alternativo para o Teorema 2.8}

Vamos exibir agora uma prova alternativa para o Teorema 2.8, cujo propósito é servir como um prenúncio do que faremos em capítulos posteriores. Para isso, vamos precisar da definição que segue. Seja $\mathbb{K}$ um sgn e $L$ uma parte de $P$. Dizemos que $L$ é afim-independente se o conjunto

$\triangleright \quad\left\{\chi^{\rho u} \mid u \in L\right\}$

é afim-independente ${ }^{1}$. A prova induzirá um algoritmo para determinar um empacotamento de $\mathbb{K}$. A ideia do algoritmo consiste em manter, a cada iteração, uma parte arbórea $B$ de $c^{+}$tal que $(B, 1)$ é positivo e uma parte $L$ de $\Lambda \mathbb{K}^{B}$ que é afim-independente. A cada iteração um dentre os conjuntos, $B$ e $L$, é estendido. Ou $B$ é estendido através da adição de um arco $a$ de tal forma que $B \cup\{a\}$ é arbóreo e $(B \cup\{a\}, 1)$ é positivo, ou $L$ é estendido através da adição de um núcleo $w \in \Lambda \mathbb{K}^{B}$ tal que $L \cup\{w\}$ é afim-independente.

Lema 2.12 Sejam $\mathbb{K}$ um sgn inteiro e não-trivial, $J:=B \cup\{e\}$ uma parte arbórea de $c^{+}$, boa para $\check{P}$, e L uma parte de $\Lambda \mathbb{K}^{B} \backslash \check{P}$ tais que

(i) $\lambda\left(\mathbb{K}^{B}\right) \geqslant \lambda \mathbb{K}-1$,

(ii) $\lambda\left(\mathbb{K}^{J}\right)=\lambda \mathbb{K}-2$,

(iii) e evita $L$.

Então existe $w \in P$ tal que

\footnotetext{
${ }^{1}$ Um conjunto finito $X$ de vetores em $\mathbb{R}^{n}$ é afim-independente se para todo $\lambda \in \mathbb{R}^{X}, \sum_{x \in X} \lambda_{x} x=0$ e $\sum_{x \in X} \lambda_{x}=0$ implicam que $\lambda=\overrightarrow{0}$.
} 

(i) $w \notin L \cup \check{P}$,
(ii) $w \in \Lambda \mathbb{K}^{B}$,e
(iii) se L é afim-independente, então $L \cup\{w\}$ é afim-independente

Prova. Existe, devido à (2.3ii), $w \in P$ tal que $c^{J}(w)=\lambda \mathbb{K}-2$ o qual, por sua vez, implica que $d_{J}(w) \geqslant 2$ e, portanto, $w \notin \check{P}$; ademais, $e$ evita $L$ e entra em $w$, donde $w \notin L$, o que prova (2.4i). No entanto, (2.3i) implica que $c^{B}(w)=\lambda \mathbb{K}-1$, o que prova (2.4ii). Para a prova de (2.4iii), note primiero que $c^{B}(\ell)=\lambda \mathbb{K}-1$ para todo $\ell \in L$. Suponha que $L$ é afim-independente e seja $L^{\prime}:=$ $L \cup\{w\}$. Suponha, por contradição, que $L^{\prime}$ não é afim-independente. Como $L$ é afim-independente, então $\chi^{\rho w}$ é uma combinação afim de $L$. Então existe $\delta: L \rightarrow \mathbb{R}$ tal que

$$
\sum_{u \in L} \delta_{u}=1 \quad \text { e } \quad \chi^{\rho w}=\sum_{u \in L} \delta_{u} \chi^{\rho u} .
$$

Segue daí que

$$
\lambda-2=c^{J}(w)=\sum\left[\delta_{u} c^{J}(u) \mid u \in L\right]=\sum\left[\delta_{u}(\lambda-1) \mid u \in L\right]=\lambda-1,
$$

o que é uma contradição. Isto estabelece (2.4iii) e completa a prova do lema.

Proposição 2.13 Seja $\llbracket$ um sgn inteiro e não-trivial, B uma parte arbórea de $c^{+}$, boa para $\check{P}$, tal que $(B, 1)$ é positivo, e L uma parte afim-independente de $\left\{u \in P \backslash \check{P} \mid c^{B}(u)=\lambda \mathbb{K}-1\right\}$. Se free $B \neq$ $\emptyset$, então existe $a \in c^{+}$tal que

$\triangleright$ a evita $L, e$

$\triangleright B \cup\{a\}$ é arbóreo,

e para o qual vale uma das alternativas:

(i) $\lambda \mathbb{K}^{B \cup\{a\}} \geqslant \lambda \mathbb{K}-1$, ou

(ii) existe $w \in P$ tal que $c^{B}(w)=\lambda \mathbb{K}-1, w \notin L e L \cup\{w\}$ é afim-independente.

Prova. Observe primeiro que $L \subseteq\left\{u \in P \mid c^{B}(u)=\lambda \mathbb{K}-1\right\}=: R$ e $(B, 1)$ é positivo, donde, pela Proposição 2.6 e pelo Lema 2.7, existe $a \in c^{+}$que evita $R$ e, portanto, evita $L$. Suponha que não vale (i). Neste caso, o Lema 2.12 implica (ii).

Prova do Teorem 2.8 Eis, então, uma prova alternativa para o Teorema 2.8: tome uma parte arbórea $B$ de $c^{+}$, boa para $\check{P}$, e uma parte afim-independente $L$ de $\Lambda \mathbb{K}^{B} \backslash \check{P}$ tais que

$\triangleright(B, 1)$ é postivo, e

$\triangleright|B|+|L|$ é máximo.

Como $|B|+|L|$ é máximo, então, de acordo com a Proposição $2.13, B$ cobre $P$, donde $\lambda \mathbb{K}^{B}=$ $\lambda \mathbb{K}-1$, o que estabelece o Teorema 2.8 .

A discussão precedente sugere o seguinte algoritmo para determinar um empacotamento de um sgn $\mathbb{K}$ : 


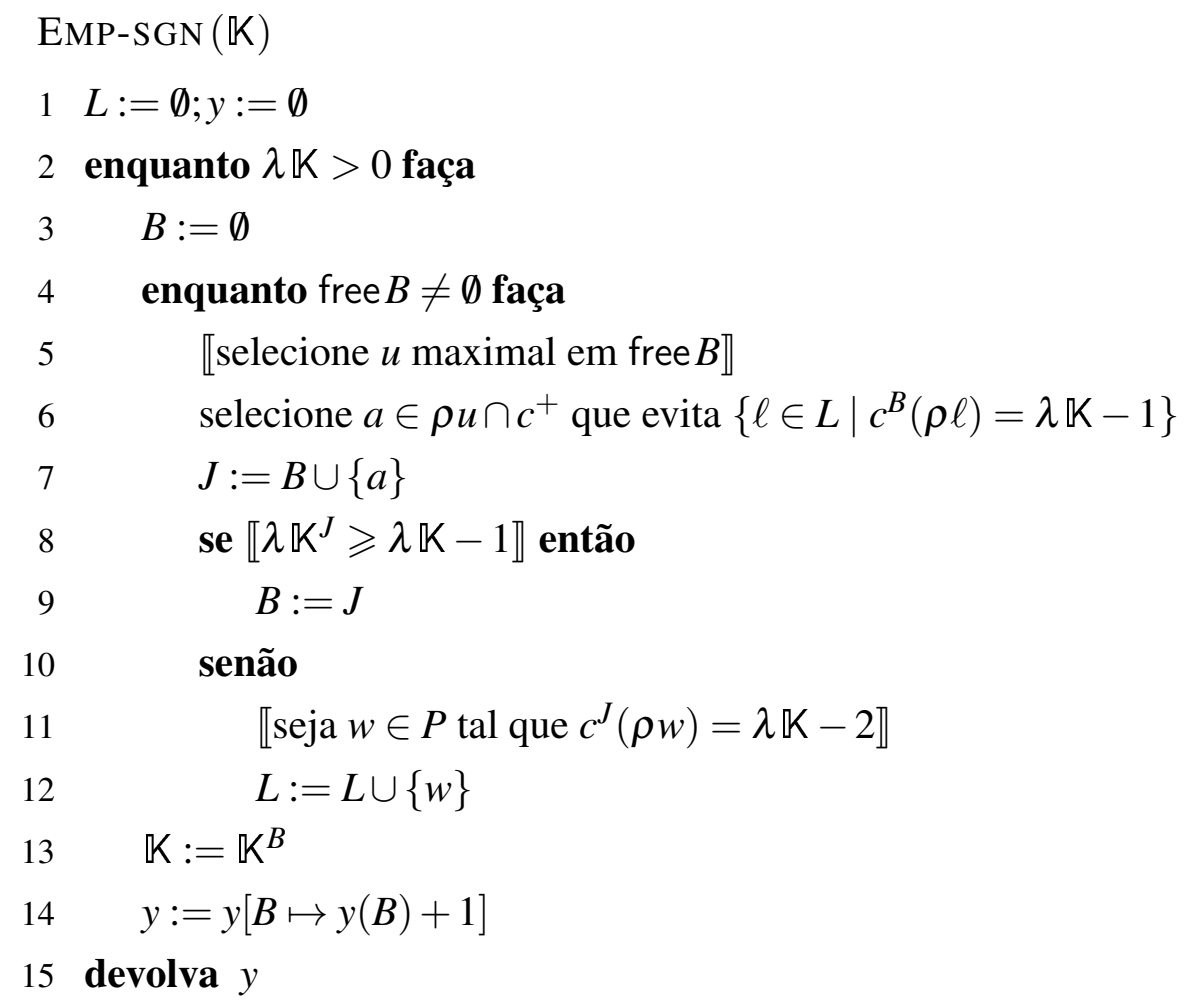

\section{Capacidade de um conjunto de arcos}

Vamos, agora, definir a noção de capacidade de um conjunto de arcos. A ideia aqui é a seguinte: dado um sgn $\mathbb{K}$ e um conjunto de $\operatorname{arcos} B$ definir um número $\varepsilon \geqslant 0$ tal que $\lambda\left(\mathbb{K}^{\varepsilon B}\right) \geqslant \lambda \mathbb{K}-\varepsilon$; um tal $\varepsilon$ naturalmente satisfaz $0 \leqslant \varepsilon \leqslant \min \{c(a) \mid a \in B\}$. Seja $u$ núcleo em $P$. É claro que $u$ é relevante se $B$ cobre $u$. Assim, suponha que $u \in \operatorname{cov} B$. Se $d_{B}(u)=1$, então $c^{\varepsilon B}(u)=c(u)-\varepsilon \geqslant \lambda-\varepsilon$. Se $d_{B}(u) \geqslant 2$, então

$$
\lambda-\varepsilon \leqslant c^{\varepsilon B}(u)=c(u)-\varepsilon d_{B}(u)
$$

se e só se

$$
\varepsilon \leqslant \frac{c(u)-\lambda}{d_{B}(u)-1}
$$

Esta discussão motiva as definições que seguem. Um núcleo $u \in P$ é especial para $B$ se $d_{B}(u) \geqslant 2$. A capacidade de um núcleo $u$, especial para $B$, é o número

$$
\triangleright \quad \operatorname{cap}(u ; B):=\operatorname{cap}_{\mathbb{K}}(u ; B):=\frac{c(u)-\lambda}{d_{B}(u)-1} .
$$

A capacidade de $B$, denotada por $\alpha_{\mathbb{K}}(B)$, ou simplesmente $\alpha B$, é o número

$$
\min (\{c(a) \mid a \in B\} \cup\{\operatorname{cap}(u ; B) \mid u \in P \text { é especial para } B\}) .
$$

O próximo lema, embora trivial, resume algumas propriedades importantes de função capacidade.

Lema 2.14 Seja $\mathbb{K}$ um sgn não-trivial, e B uma parte de $c^{+}$, boa para $\Lambda \mathbb{K}$. Então

(i) $\lambda \mathbb{K}^{(\alpha B) B} \geqslant \lambda \mathbb{K}-\alpha B, e$ 
(ii) se B é uma cobertura de $P$, então $\lambda\left(\mathbb{K}^{\varepsilon B}\right)=\lambda \mathbb{K}-\varepsilon$ para cada $0 \leqslant \varepsilon \leqslant \alpha B$.

Prova. Note que (ii) segue de (i) da definição (2.5). Para a prova de (i), escreva $\mathbb{K}^{\prime}:=\mathbb{K}^{(\alpha B) B}$. Tome $u \in P$. Se $d_{B}(u) \leqslant 1$, então $B$ bom para $\Lambda$ implica $c^{\prime}(u) \geqslant \lambda-\alpha B$. Suponha que $d_{B}(u) \geqslant 2$. Como $\alpha B \leqslant \operatorname{cap}(u ; B)$, então $c^{\prime}(u) \geqslant \lambda-\alpha B$, devido à definição (2.5). Isto estabelece (i).

Lema 2.15 Seja $\mathbb{K}$ um sgn, $B \subseteq E$ tal que $(B, \alpha B)$ é positivo, e $R:=\Lambda \cap \operatorname{cov} B$. Então

(i) $(R, P, B, \alpha B)$ é evitável, $e$

(ii) se $a \in c^{+}$evita $R$, então $(B \cup\{a\}, \alpha(B \cup\{a\}))$ é também positivo.

Prova. A propriedade (n.0) é trivial, assim como (n.2) e (n.3). Além disso, $B$ bom para $\Lambda$ e $R \subseteq \Lambda$ implicam (n.4). Resta, pois, provar (n.1). Primeiro observe que $(B, \alpha B)$ positivo implica em $B$ bom para $\Lambda$. Sejam $u, w \in R$ tais que $u \bowtie w$. Como $R \subseteq \Lambda$, então

$$
c(u)+c(w)=c(u \vee w)+c(u \wedge w) .
$$

Seja $a \in B$. Como $(B, \alpha B)$ é positivo, então $c(a)>0$ o que, aliado ao Lema 2.3i, implica que

$(*) \quad d_{\{a\}}(u)+d_{\{a\}}(w)=d_{\{a\}}(u \vee w)+d_{\{a\}}(u \wedge w)$.

A prova de que $R$ é $\bowtie$-fechado é por indução em $|B|$. O caso $B=\emptyset$ é trivial. Assim, suponha que $B \neq \emptyset$. Seja $e \in B$, defina $B^{\prime}:=B \backslash\{e\}$ e seja $R^{\prime}:=\Lambda \cap \operatorname{cov} B^{\prime}$. É claro que $\left(B^{\prime}, \alpha B^{\prime}\right)$ é positivo, donde, por hipótese de indução, $R^{\prime}$ é $\bowtie$-fechado. Sejam $u, w \in R$ tais que $u \bowtie w$. Se $u, w \in R^{\prime}$, então não há nada a provar. Se $u, w \in R \backslash R^{\prime}$, então $e$ cobre $u$ e $w$ o que, aliado à (*), implica que $e$ cobre $u \vee w$ e $u \wedge w$ e, portanto, $u \vee w$ e $u \wedge w$ estão ambos em $R$. Daqui para frente, vamos admitir que exatamente um, dentre $u$ e $w$, digamos $u$, está em $R \backslash R^{\prime}$. Desta forma, $w \in R^{\prime}$ e, assim, um dos $\operatorname{arcos}$ em $B^{\prime}$, digamos $f$, é tal que $f \in \rho w$. Ademais, $e \in \rho u \backslash \rho w$ pois $B$ é bom para $\Lambda$. Como $u \notin R^{\prime}$, então temos também que $f \notin \rho u$. Agora, de $e \in \rho u \backslash \rho w$ combinado com (*) vem que $e \in \rho(u \vee w)$ ou $e \in \rho(v \wedge w)$. Se $e \in \rho(u \vee w)$ então $f \notin \rho(u \vee w)$ pois $B$ é bom para $\Lambda$. Mas $f \in \rho w \backslash \rho u, f \notin \rho(u \vee w)$ e $(*)$ fornecem $f \in \rho(u \wedge w)$. Portanto, $u \vee w, u \wedge w \in R$, como queríamos. O caso remanescente - quando $e \in \rho(u \wedge w)$ - segue de forma análoga. Isto prova (n.1). Finalmente, o item (ii) é trivial, por isso omitimos sua prova.

Usando o Lema 2.15, e imitando a prova do Teorema 2.8, podemos derivar o teorema que segue.

Teorema 2.16 Seja $\mathbb{K}$ um sgn não-trivial.

Então existe uma cobertura $B$ de $P$, que é boa para $\check{P}$, tal que $\alpha B>0$ e $\lambda \mathbb{K}^{(\alpha B) B}=\lambda \mathbb{K}-\alpha B$.

Observe que a cobertura garantida pelo teorema é, na verdade, boa para $\check{P} \cup \Lambda \mathbb{K}$. O próximo lema constitui uma forma alternativa de derivar o Teorema 2.16.

Lema 2.17 Seja $\mathbb{K}$ um $\mathbf{s g n}$ não-trivial, B uma parte de E tal que $(B, \alpha B)$ é positivo, $e$

$$
R:=\left\{u \in P \mid c^{(\alpha B) B}(u)=\lambda \mathbb{K}-\alpha B\right\} .
$$


Então

(i) $(R, P, B, \alpha B)$ é evitável, $e$

(ii) se $a \in c^{+}$evita $R$, então $(B \cup\{a\}, \alpha(B \cup\{a\}))$ é também positivo.

Prova. Eis a prova de (i). As propriedades $(n .0),(n .2),(n .3)$ e $(n .4)$ seguem trivialmente. Resta, assim, verificar (n.1). Vamos provar que $R$ é $\bowtie$-fechado. Para abreviar, escreva $\varepsilon:=\alpha B$. Sejam $u, w \in R \operatorname{com} u \bowtie w$. Então

$$
\lambda-\varepsilon+\lambda-\varepsilon=c^{\varepsilon B}(u)+c^{\varepsilon B}(w) \geqslant c^{\varepsilon B}(u \vee w)+c^{\varepsilon B}(u \wedge w) \geqslant \lambda-\varepsilon+\lambda-\varepsilon .
$$

A primeira igualdade é válida pois $u$ e $w$ estão em $R$. A primeira desigualdade segue do Lema 2.2. A segunda desigualdade é verdadeira, pois $(B, \varepsilon)$ é positivo. Assim, vale a igualdade em todas as passagens e, por conseguinte, $u \vee w, u \wedge w \in R$. Isto estabelece que $R$ é $\bowtie$-fechado e, portanto, também (n.1). Finalmente, observe que (ii) segue diretamente da definição de capacidade.

Note, entretanto, que derivar a versão minimax para capacidades arbitrárias, ou seja, estabelecer que um sgn $\mathbb{K}$ empacota, garantindo que o número de coberturas é polinomial no número de arcos não é imediato. Exibimos, abaixo, um algoritmo baseado na derivação do Teorema 2.16. Surpreendentemente, como veremos no contexto de um arcabouço mais geral, este algoritmo produz um empacotamento com um número de coberturas polinomial (linear) no número de arcos. No algoritmo que segue podemos tomar, a cada iteração,

$$
R_{B}:=\Lambda(\mathbb{K}) \cap \operatorname{cov} B \quad \text { ou } \quad R_{B}:=\left\{r \in P \mid c^{(\alpha B) B}(\rho r)=\lambda \mathbb{K}-\alpha B\right\} .
$$

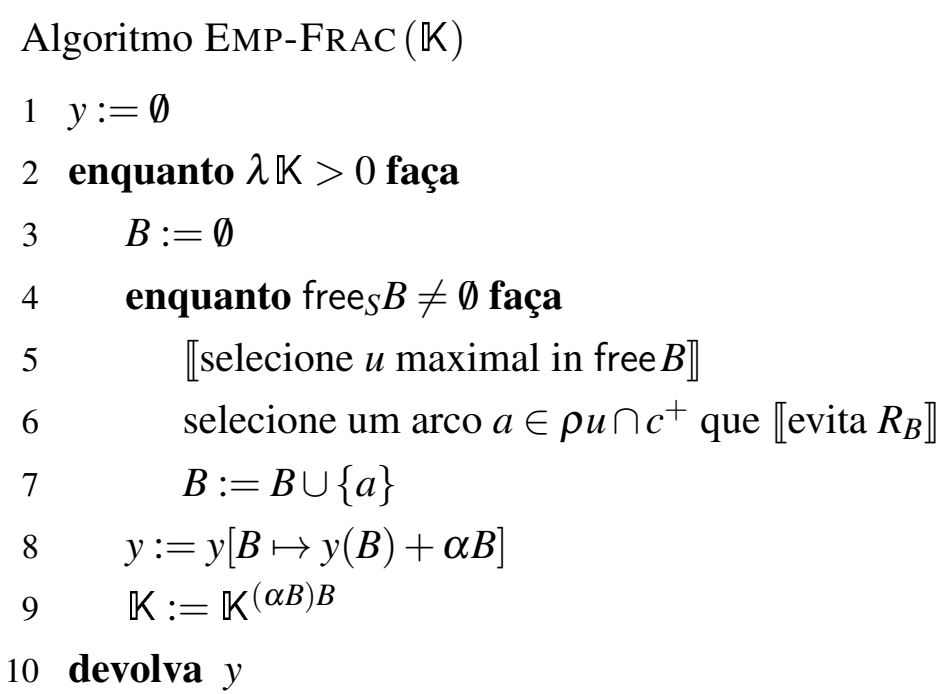

O próximo lema, aliado ao Lema 2.14, permite produzir uma prova alternativa do Teorema 2.8 .

Lema 2.18 Seja $\mathbb{K}$ um sgn inteiro, $B$ uma parte de $E$, e $R:=\left\{u \in P \mid c^{(\alpha B) B}(\rho u)=\lambda \mathbb{K}-(\alpha B) B\right\}$. Se $(B, \alpha B)$ é positivo, e $\alpha B \geqslant 1$ então $(B \cup\{a\}, \alpha(B \cup\{a\})$ é positivo e $\alpha(B \cup\{a\}) \geqslant 1$ para cada arco $a \in c^{+}$que evita $R$.

Prova. Suponha que $(B, \alpha B)$ é positivo e $\alpha B \geqslant 1$. Seja $a \in c^{+}$tal que $a$ evita $R$. Seja $\varepsilon:=\alpha B$ e $B^{\prime}:=B \cup\{a\}$. O Lema 2.17 implica que $(B \cup\{a\}, \alpha(B \cup\{a\}))$ é positivo. Note que $c \geqslant 1$. Logo, é suficiente mostrar que 
$\rightsquigarrow \quad \operatorname{cap}\left(z ; B^{\prime}\right) \geqslant 1$ para cada $z \in P \operatorname{com} d_{B^{\prime}}(z) \geqslant 2$.

Seja, então, $z \in P \operatorname{com} d_{B^{\prime}}(z) \geqslant 2$. Se $a$ evita $z$, então $d_{B}(z)=d_{B^{\prime}}(z)$ e, daí, $\operatorname{cap}\left(z ; B^{\prime}\right)=\operatorname{cap}(z ; B) \geqslant$ 1. Agora, admita que $a$ cobre $z$. O arco $a$ evita $R$ mas cobre $z$, donde $c^{\varepsilon B}(\rho z)>\lambda-\varepsilon$. Se $d_{B^{\prime}}(z)=2$, então $c(z)-\varepsilon=c^{\varepsilon B}(\rho z)>\lambda-\varepsilon$ o que acarreta que $c(z)>\lambda$. Daí, a integralidade de $c$ e $\lambda$ implica que $\operatorname{cap}\left(z ; B^{\prime}\right)=c(z)-\lambda \geqslant 1$. Finalmente, suponha que $d_{B}(z) \geqslant 3$. Ora,

$$
\lambda-\varepsilon<c^{\varepsilon B}(\rho z)=c(z)-\varepsilon d_{B}(z)
$$

e, de $\varepsilon \geqslant 1$ vem que $c(z)-\lambda>d_{B}(z)-1$. Por integralidade,

$$
\operatorname{cap}\left(z ; B^{\prime}\right)=\frac{c(z)-\lambda}{d_{B^{\prime}}(z)-1} \geqslant 1
$$

como queríamos.

Finalmente, o Teorema 2.16, aliado a um argumento de compacidade, fornece o seguinte teorema.

Teorema 2.19 Seja $\mathbb{K}$ um $\operatorname{sgn} e \mathcal{B}$ a família das coberturas de $P$, boas para $\check{P}$. Então

$$
\max \left\{\sum_{B \in \mathcal{B}} y(B) \mid y \geqslant \overrightarrow{0} ; \sum_{B \in \mathcal{B}} y(B) \chi^{B} \leqslant c\right\}=\lambda \mathbb{K} .
$$




\section{Capítulo 3}

\section{Sistemas generalizados de núcleos com a propriedade do cruzamento}

Neste capítulo, vamos introduzir um novo tipo de arcabouço, obtido de um sistema generalizado de núcleos pela adição de um axioma que permitirá definir uma operação de descruzamento. Esta operação possibilitará, como exibiremos no Capítulo 5, fornecer um melhor limitante superior para o tamanho de um empacotamento. Este novo arcabouço ainda contém, como uma de suas instâncias, os sistemas de bi-conjuntos intersectantes e, portanto, os resultados aqui obtidos podem ser aplicados, para por exemplo, deduzir propriedades a respeito do empacotamento de arborescências geradoras em digrafos, e também como veremos em um capítulo posterior, sobre o empacotamento de ramificações em digrafos.

\subsection{Sistemas generalizados de núcleos com a propriedade do cruzamento}

Eis a definição formal do arcabouço deste capítulo. Seja $\mathbb{K}$ um sistema generalizado de núcleos. Dizemos que $\mathbb{K}$ satisfaz a propriedade do cruzamento se

( $\rho .4)$ para cada $u, \ell, k \in P$ : se $\ell \bowtie u, k \bowtie(u \vee \ell)$ e $k \bowtie \ell$, então $k \bowtie u$.

$\mathrm{Na}$ falta de um nome melhor vamos denotar um tal sgn pela sigla sgn-c.

Sequências propriamente intersectantes Durante este capítulo vamos ter que lidar com sequências de núcleos. Lembre-se que $\emptyset$ denota a sequência vazia. Fixe um sgn $\mathbb{K}$. Para uma sequência $\mathbf{f} \subseteq P$ e um núcleo $v$ em $P$, escrevemos $\mathbf{f} \cdot v$ para denotar a sequência obtida de $\mathbf{f}$ pela adição de $v$ como último elemento, e dizemos, neste caso, que $v$ é um núcleo de $\mathbf{f} \cdot v$, ou está em $\mathbf{f} \cdot v$. Definimos, recursivamente, para cada $u, v \in P$ e cada sequência $\mathbf{f} \subseteq P$ :

$$
\begin{aligned}
& \text { - } u \vee \emptyset:=u \\
& \text { - } u \vee(\mathbf{f} \cdot v):=(u \vee \mathbf{f}) \vee v
\end{aligned}
$$


Note que $u \vee \mathbf{f}$ pode não estar bem definido. A próxima definição estabelece uma restrição sobre $\mathbf{f}$ que garantirá que $u \vee \mathbf{f}$ faz sentido. Seja $M$ uma parte de $P, \mathbf{f} \subseteq M$ uma sequência e $u \in P$. Dizemos que f é uma sequência propriamente intersectante em relação $a u$, ou simplesmente um $u$-spi, se $\mathbf{f}=\emptyset$ ou existe uma sequência $\mathbf{f}^{\prime}$ e um núcleo $\ell \in P$ tais que

$\triangleright \quad \mathbf{f}=\mathbf{f}^{\prime} \cdot \ell, \quad \ell \bowtie u \vee \mathbf{f}^{\prime} \quad$ e $\quad \mathbf{f}^{\prime}$ é uma $u$-spi.

\subsection{Um procedimento de descruzamento}

É apropriado, agora, relembrar algumas definições. Seja $\mathbb{K}$ um sgn, $L$ uma parte de $P$ e $u$ um dos núcleos de $\mathbb{K}$. Dizemos que $L$ é laminar se $u, w \in L$ implica $u \bowtie w$. Denotamos por $L_{u}$ o conjunto dos núcleos maximais $\ell$ de $L$ para os quais $\ell \bowtie u$.

Lema 3.1 Seja $\mathbb{K}$ um sgn-c, I uma parte $\bowtie$-fechada de $P$, L um subconjunto laminar de I, e u um núcleo em I. Então existe uma $u$-spi f $\subseteq L_{u}$ tal que

$$
u \vee \mathbf{f} \in I \quad e \quad L \cup\{u \vee \mathbf{f}\} \text { é laminar. }
$$

Prova. Vamos, primeiro, provar o seguinte fato:

Fato 3.1.1 Para cada $M \subseteq L_{u}$ existe uma $u$-spi $\mathbf{f} \subseteq M$ tal que

(*) $\quad$ para cada $m \in M: m \bowtie(u \vee \mathbf{f}) \quad$ e $\quad u \vee \mathbf{f} \in I$.

Prova. A prova é uma indução em $|M|$. Para $M=\emptyset$, o resultado é óbvio. Suponha, assim, que $M \neq \emptyset$. Seja $\ell \in M$ e $M^{\prime}:=M \backslash\{\ell\}$. Por hipótese de indução, existe uma $u$-spi $\mathbf{f}^{\prime} \subseteq M^{\prime}$ para a qual

para cada $m \in M^{\prime}: m \bowtie u \vee \mathbf{f}^{\prime} \quad$ e $\quad u \vee \mathbf{f}^{\prime} \in I$.

Se $\ell \bowtie\left(u \vee \mathbf{f}^{\prime}\right)$, então não há mais nada a provar. Suponha $\ell \bowtie\left(u \vee \mathbf{f}^{\prime}\right)$ e seja $\mathbf{f}:=\mathbf{f}^{\prime} \cdot \ell$. É evidente que f é uma $u$-spi. O núcleo $u \vee \mathbf{f}$ está em $I$, pois $I$ é $\bowtie$-fechado. Além disso, $\ell \bowtie(u \vee \mathbf{f})$. Assim, seja $k \in M^{\prime}$, e suponha, por contradição, que $k \bowtie(u \vee \mathbf{f})$. Ponha $w^{\prime}:=u \vee \mathbf{f}^{\prime}$. Então $k \bowtie w^{\prime}$ pois $k \in M^{\prime}$. Finalmente,

$$
\ell \bowtie w^{\prime}, \quad k \bowtie w^{\prime} \vee \ell, \quad \text { e } \quad \ell \bowtie k,
$$

combinado com $(\rho .4)$, fornece $k \bowtie w^{\prime}$ o qual, por sua vez, contraria $k \not \downarrow w^{\prime}$.

O Fato 3.1.1 implica que existe uma $u$-spi f tal que $u \vee \mathbf{f} \in I$ e $m \bowtie(u \vee \mathbf{f})$ para cada $m \in L_{u}$. O próximo fato estabelece que $L \cup\{u \vee \mathbf{f}\}$ é, de fato, laminar.

Fato 3.1.2 $L \cup\{u \vee \mathbf{f}\}$ é laminar.

Prova. De fato, suponha que este não é o caso. Então existe $k \in L$ tal que $k \bowtie(u \vee \mathbf{f})$. Afirmamos que $k \bowtie u$. Suponha, por contradição, que $k \bowtie u$. Seja $\mathbf{f}^{\prime}$ um prefixo mínimo de $\mathbf{f}$ dotado das seguintes propriedades: 


$$
k \bowtie\left(u \vee \mathbf{f}^{\prime}\right) \quad \text { e } \quad k \bowtie\left(u \vee \mathbf{f}^{\prime}\right) \vee \ell,
$$

onde $\ell \in L_{u}$ é tal que $\mathbf{f}^{\prime} \cdot \ell$ é também um prefixo de f. Um tal prefixo existe uma vez que $k \bowtie u$ e $k \bowtie(u \vee \mathbf{f})$. De $k, \ell \in L$ vem que $k \bowtie \ell$. Ponha $w^{\prime}:=u \vee \mathbf{f}^{\prime}$ e, assim, $k \bowtie w^{\prime}$. Ora, $\ell \bowtie w^{\prime}$ pois f é uma $u$-spi. Por hipótese, $k \bowtie w^{\prime} \vee \ell$. Agora, ( $\left.\rho .4\right)$ implica $k \bowtie w^{\prime}$, uma contradição. Logo, $k \bowtie u$ e, pelo Fato 3.1.1, $k \notin L_{u}$ o qual, por sua vez, implica que existe $m \in L_{u} \operatorname{com} k \prec m$. Como $m \in L_{u}$, então o Fato 3.1.1 implica que $m \bowtie(u \vee \mathbf{f})$. Assim, vale exatamente uma das alternativas
(a) $m \sim(u \vee \mathbf{f}) \quad$ ou
(b) $m \nsim(u \vee \mathbf{f})$ e $m^{\downarrow} \cap(u \vee \mathbf{f})^{\downarrow}=\emptyset$.

Suponha que vale a alternativa (a). Se $m \preccurlyeq u \vee \mathbf{f}$, então $k \prec u \vee \mathbf{f}$, o que contraria $k \bowtie u \vee \mathbf{f}$. Se, por outro lado, $u \vee \mathbf{f} \prec m$, então $u \prec m$, o que contraria $m \bowtie u$. Assim, não vale a alternativa (a), ou seja, $m \nsim u \vee f$. Finalmente, de $k \bowtie(u \vee \mathbf{f})$ e $k \prec m$ segue $(u \vee \mathbf{f}) \wedge k \in m^{\downarrow} \cap(u \vee \mathbf{f})^{\downarrow}$, o que é uma contradição e, desta forma, a alternativa (b) também não vale. Concluímos, assim, que um tal núcleo $k$ não existe e, portanto, $L \cup\{u \vee \mathbf{f}\}$ é laminar.

Isto completa a prova do lema.

É claro que a prova do lema precedente induz um algoritmo que recebe um sgn $\mathbb{K}$, uma parte laminar $L \subseteq I$, e $u \in I$, onde $I$ é uma parte $\bowtie$-fechada de $P$, e devolve $w \in I$ tal que $L \cup\{w\}$ é laminar. Observe, entretanto, que $w$ pode ser um elemento de $L$.

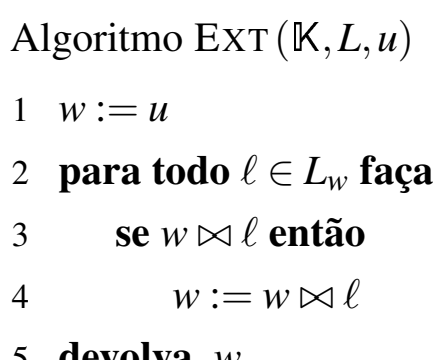

\subsection{Um algoritmo alternativo}

Nesta seção, vamos exibir um algoritmo alternativo. que produz um empacotamento em um sgn-c inteiro. Como é de se esperar, tal algoritmo, em geral, não é polinomial. No entanto, em sgn-cs não-capacitados, o algoritmo tem complexidade polinomial. É importante destacar que este algoritmo é uma generalização de um algoritmo de Gabow e Manu [GM98] para a versão nãocapacitada do empacotamento de arborescências geradoras em digrafos.

Vamos fazer uma descrição informal do algoritmo para ressaltar suas ideias fundamentais. Cada iteração do algoritmo começa com dois objetos: (1) uma parte $B$ de $c^{+}$, boa para $\check{P}$, tal que $(B, 1)$ é positivo e $(2)$ uma parte laminar $L$ de $P \backslash \check{P}$ cujos elementos são justos, conceito este que definiremos posteriormente. Cada iteração seleciona um arco $a$ que evita $L$. Se $(B \cup\{a\}, 1)$ é positivo, então começamos uma nova iteração com $B \cup\{a\}$ no lugar de $B$. Caso contrário, usamos o Lema 3.1 para determinar um núcleo $w$ que está fora de $L \cup \check{P}$ e para o qual $L \cup\{w\}$ é laminar e começamos uma nova iteração com $L \cup\{w\}$ no lugar de $L$. 
Lema 3.2 Sejam $\mathbb{K}$ um sgn-c, $J:=B \cup\{e\}$ uma parte arbórea, boa para $\check{P}$, e L uma parte laminar de $\left(\Lambda \mathbb{K} \cup \Lambda \mathbb{K}^{B}\right) \backslash \check{P}$ tais que

(i) $\lambda\left(\mathbb{K}^{B}\right) \geqslant \lambda \mathbb{K}-1$,

(ii) $\lambda\left(\mathbb{K}^{J}\right)=\lambda \mathbb{K}-2, e$

(iii) e evita $\left\{\ell \in L \mid c^{B}(\ell)=\lambda \mathbb{K}-1\right\}$.

Então existe $w \in P$ tal que
(i) $w \notin L \cup \check{P}$,
(ii) $L \cup\{w\}$ é laminar, $e$
(iii) $c^{B}(w)=\lambda \mathbb{K}-1$.

Prova. Observe, primeiro, que (3.2i) e (3.2iii) implicam que $c^{J}(\ell) \in\{\lambda \mathbb{K}, \lambda \mathbb{K}-1\}$ para todo $\ell \in L$. Seja $X:=\left\{u \in P \mid c^{J}(u)=\lambda \mathbb{K}-2\right\}$. Claramente, por (3.2.ii), $X$ é não-vazio donde o Lema 2.1 implica que existe $u \in X$ tal que para cada $\ell \in L_{u}$ e $z \in P$, se $z \prec \ell$, então $z \notin X$, ou equivalentemente,

$$
c^{J}(u)=\lambda \mathbb{K}-2, \mathrm{e}
$$

para cada $\ell \in L_{u}, z \in P$ : se $z \prec \ell$ então $c^{J}(z) \geqslant \lambda \mathbb{K}-1$.

Note que $c^{J}(u)=\lambda \mathbb{K}-2$ implica $d_{J}(u) \geqslant 2$; mas, $J$ é bom para $\check{P}$ e, daí, $u \notin \check{P}$. Agora, seja $\mathbf{f} \subseteq L_{u}$ uma $u$-spi tal que $L \cup\{u \vee \mathbf{f}\}$ é laminar, cuja existência é garantida pelo Lema 3.1. Vamos provar que $u \vee \mathbf{f} \notin L$. Devido à (3.2.iii), é suficiente estabelecer que $c^{J}(u \vee \mathbf{f})=\lambda \mathbb{K}-2$. A prova disto é por indução no comprimento de $\mathbf{f}$. Se $\mathbf{f}=\emptyset$, não há nada a provar. Admita, pois, que $\mathbf{f}=\mathbf{f}^{\prime} \cdot \ell$ para alguma $u$-spi $\mathbf{f}^{\prime}$ e algum $\ell \in L_{u}$. Por hipótese de indução, $c^{J}\left(u \vee \mathbf{f}^{\prime}\right)=\lambda \mathbb{K}-2$. Como de hábito, escreva $\lambda:=\lambda \mathbb{K}$. Dois casos necessitam consideração:

\section{Caso $1 J$ evita $\ell$}

Neste caso, $c^{J}(\ell)=\lambda$. Ademais, $J$ evita $(u \vee \mathbf{f}) \wedge \ell$ pois $J$ é arbóreo. Segue daí que $c^{J}((u \vee$ f) $\wedge \ell) \geqslant \lambda$. Então

$$
\begin{aligned}
& \lambda-2+\lambda=c^{J}\left(u \vee \mathbf{f}^{\prime}\right)+c^{J}(\ell) \\
& \geqslant c^{J}\left(\left(u \vee \mathbf{f}^{\prime}\right) \vee \ell\right)+c^{J}\left(\left(u \vee \mathbf{f}^{\prime}\right) \wedge \ell\right) \\
& \geqslant \lambda-2+\lambda,
\end{aligned}
$$

donde $c^{J}(u \vee \mathbf{f})=\lambda-2$

Caso $2 J$ cobre $\ell$.

Neste caso, em virtude de (3.2iii), temos que $c^{J}(\ell)=\lambda-1$. Como $(u \vee \mathbf{f}) \wedge \ell \prec \ell$, então a escolha de $u$ e $\ell \in L_{u}$ acarretam $c^{J}((u \vee \mathbf{f}) \wedge \ell) \geqslant \lambda-1$. Usando submodularidade obtemos

$$
\begin{aligned}
& \lambda-2+\lambda-1=c^{J}\left(u \vee \mathbf{f}^{\prime}\right)+c^{J}(\ell) \\
& \geqslant c^{J}\left(\left(u \vee \mathbf{f}^{\prime}\right) \vee \ell\right)+c^{J}\left(\left(u \vee \mathbf{f}^{\prime}\right) \wedge \ell\right) \\
& \geqslant \lambda-2+\lambda-1,
\end{aligned}
$$


e, uma vez mais, $c^{J}(u \vee \mathbf{f})=\lambda-2$.

Agora, $c^{B}(u \vee \mathbf{f})=\lambda-1$ devido à (3.2.i), o que prova (3.3iii); ademais, $u \vee \mathbf{f} \notin \check{P}$ pois $u \preccurlyeq u \vee \mathbf{f}$ e $u \notin \check{P}$. Isto completa a prova do lema.

Proposição 3.3 Seja $\llbracket$ um sgn-c inteiro e não-trivial, $B$ uma parte arbórea de $c^{+}$, boa para $\check{P}$, tal que $\lambda \mathbb{K}^{B} \geqslant \lambda \mathbb{K}-1$, e L uma parte laminar de $\left(\Lambda \mathbb{K} \cup \lambda \mathbb{K}^{B}\right) \backslash \check{P}$. Se free $B \neq \emptyset$, então existe $a \in c^{+}$ tal que

$\triangleright$ a evita $R_{1}:=\left\{\ell \in L \mid c^{B}(\rho \ell)=\lambda \mathbb{K}-1\right\}, e$

$\triangleright B \cup\{a\}$ é arbóreo e bom para $\check{P}$,

e para o qual vale uma das alternativas:

(i) $\lambda \mathbb{K}^{B \cup\{a\}} \geqslant \lambda \mathbb{K}-1$, ou

(ii) existe $w \in \Lambda \mathbb{K}^{B}$ tal que $w \notin L \cup \check{P}$ e $L \cup\{w\}$ é laminar.

Prova. Observe primeiro que $R_{1} \subseteq\left\{u \in P \mid c^{B}(u)=\lambda \mathbb{K}-1\right\}=: R$ e $(B, 1)$ é positivo donde, pela Proposição 2.6 e pelo Lema 2.7, existe $a \in c^{+}$que evita $R$ e, portanto, evita $R_{1}$. Além disso, o conjunto $B \cup\{a\}$, pelo Proposição 2.6, é arbóreo e bom para $\check{P}$. Suponha que não vale (i). Então $\lambda \mathbb{K}^{B \cup\{a\}}=\lambda \mathbb{K}-2$. Ora, o Lema 3.2 implica que existe $w \in \Lambda \mathbb{K}^{B}$ tal que $w \notin L \cup \check{P}$ e $L \cup\{w\}$ é laminar e, portanto, estabelecendo (ii).

É evidente que esta proposição implica no seguinte fato, que é um caso especial do Teorema 2.8:

Fato 3.3.1 Se $\mathbb{K}$ é um sgn-c inteiro e não-trivial, então existe uma cobertura $B$ de $P$, boa para $\check{P}$, tal que $\lambda\left(\mathbb{K}^{B}\right)=\lambda \mathbb{K}-1$.

No entanto, esta mesma proposição fornece um algoritmo alternativo, descrito no que segue, para construir uma tal cobertura. 


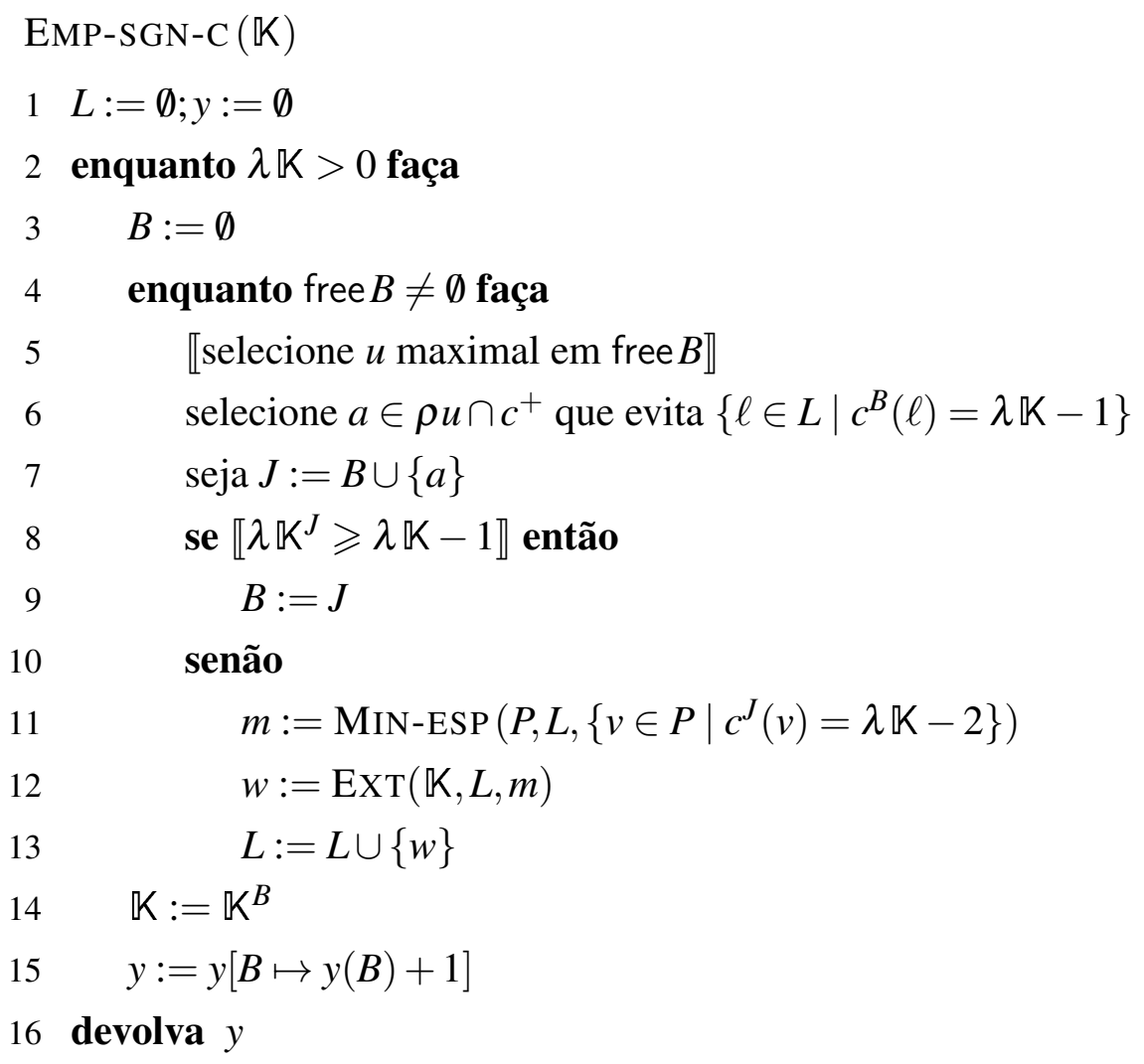




\section{Capítulo 4}

\section{Sistemas generalizados de núcleos com uma família mista}

Neste capítulo, vamos introduzir os sistemas generalizados de núcleos com uma família mista, os quais constituem uma generalização do problema do empacotamento em sgns, e provar que tal arcabouço empacota. Este arcabouço generaliza os sistemas de bi-conjuntos que satisfazem a propriedade da intersecção mista, devido a Frank e Bércsi [BF08], os quais, por sua vez, generalizam um arcabouço de Szegó [Sze01].

\subsection{Famílias mistas}

Seja $\mathbb{K}$ um sgn e $\mathcal{C}$ uma família de subconjuntos $\bowtie$-fechados de $P$. Dizemos que $\mathcal{C}$ satisfaz a propriedade da intersecção mista, ou simplesmente pim, se para cada $S, T \in \mathcal{C}$ e cada $s \in S, t \in T$ : $\triangleright \quad s \widetilde{\bowtie} t$ implica $s \wedge t \in S \cap T$.

Para encurtar, dizemos que $\mathcal{C}$ é uma família mista se $\mathcal{C}$ é uma família de subconjuntos $\bowtie$-fechados de $P$ dotada da propriedade da intersecção mista. Note que cada subfamília de uma família mista também é uma família mista. Seja $\mathcal{U}(P)$, ou simplesmente $\mathcal{U}$, a família de todos os subconjuntos $\bowtie$-fechados de $P$. Uma função $\mu: \mathcal{U} \rightarrow \mathbb{R}_{+}$é chamada de função demanda. Para cada $u \in P$, pomos

$\triangleright \quad p(u):=p_{\mu}(u):=\sum[\mu S \mid u \in S, S \in \mathcal{U}]$.

e dizemos que $p(u)$ é a demanda de $u$. O par $(\mathbb{K}, \mu)$ é denominado de um sistema generalizado de núcleos com uma família mista, ou simplesmente um sgnfm, se $\mu^{+}$é uma família mista. Vamos, tipicamente, escrever $\mathbb{F}$ para denotar um sgnfm arbitrário e, salvo dito o contrário, vamos admitir que $\mathbb{F}=(\mathbb{K}, \mu)$. Também dizemos que $\mathbb{F}$ é inteiro se $\mathbb{K}$ é inteiro e $\mu: \mathcal{U} \rightarrow \mathbb{N}$.

O problema do empacotamento É conveniente, agora, relembrar alguns conceitos. Seja $\mathbb{F}$ um sgnfm. Para cada parte $\bowtie$-fechada $S$ de $P$, seja $\mathcal{B}_{S}$ o conjunto das coberturas de $S$. Para cada $S \in \mathcal{U}$, uma função $y_{S}: \mathcal{B}_{S} \rightarrow \mathbb{R}_{+}$é um empacotamento de $S$ se 


$$
\sum\left[y_{S}(B) \mid B \in \mathcal{B}_{S}\right]=\mu S
$$

Um empacotamento é inteiro se sua imagem é um subconjunto de $\mathbb{N}$. Uma função y que atribui um empacotamento $y_{S}$ para cada $S \in \mathcal{U}$ é um empacotamento de $\mathbb{F}$, ou simplesmente um empacotamento, se

$$
\sum\left[y_{S}(B) \chi^{B} \mid S \in \mathcal{U}, B \in \mathcal{B}_{S}\right] \leqslant c .
$$

Também dizemos que y é inteiro se a imagem de $y_{S}$ é uma parte de $\mathbb{N}$ para cada $S \in \mathcal{U}$. Finalmente, dizemos que $\mathbb{F}$ empacota se $\mathbb{F}$ possui um empacotamento. Vamos enumerar os problemas que nos interessarão neste e no próximo capítulo. Para isso, seja $\mathbb{F}$ um sgnfm.

Problema 1 F empacota?

Problema 2 Se $\mathbb{F}$ empacota, então existe um empacotamento $y$ de $\mathbb{F}$ tal que $\left|y^{+}\right|$é polinomial em $|E|$ e $\left|\mu^{+}\right|$?

Problema 3 Se $\mathbb{F}$ é inteiro, então $\mathbb{F}$ possui um empacotamento inteiro?

Problema 4 Se $\mathbb{F}$ é inteiro e $\mathbb{F}$ empacota, então existe um empacotamento inteiro $y$ de $\mathbb{F}$ tal que $\left|y^{+}\right|$é polinomial em $|E|$ e $\left|\mu^{+}\right|$?

Veremos neste, e no próximo capítulo, que a resposta para todos os problemas é "sim". Neste capítulo, vamos exibir uma solução para o Problema 3 e estabelecer alguns fatos fundamentais que permitirão produzir uma solução para os demais problemas, as quais serão mostradas no Capítulo 5. Assim, no restante deste capítulo, vamos estabelecer alguns lemas — os quais serão úteis também na solução dos Problemas 1, 2, e 4 - e o teorema que produz uma solução para o Problema 3.

A próxima noção é fundamental pois ela fornecerá uma condição necessária e suficiente que garante que um sgnfm empacote. Dizemos que $\mathbb{F}$ é viável se

$\triangleright \quad$ para cada $u \in P: c(u) \geqslant p(u)$.

Eis, então, a prova da necessidade desta condição:

Lema 4.1 Se $\mathbb{F}$ é um $\operatorname{sgnfm} e \mathbb{F}$ empacota, então $\mathbb{F}$ é viável.

Prova. Seja $\mathbb{F}$ um sgnfm e suponha que $y$ é um empacotamento de $\mathbb{F}$. Então para cada $u \in P$ :

$$
\begin{aligned}
& p(u)=\sum[\mu S \mid S \in \mathcal{U}, u \in S]=\sum\left[\sum\left[y_{S}(B) \mid B \in \mathcal{B}_{S}\right] \mid S \in \mathcal{U}, u \in S\right] \\
& =\sum\left[y_{S}(B) \mid S \in \mathcal{U}, B \in \mathcal{B}_{S}, u \in S\right] \leqslant \sum\left[y_{S}(B) \mid S \in \mathcal{U}, B \in \mathcal{B}_{S}, B \cap \rho u \neq \emptyset\right] \\
& =\sum_{e \in \rho u} \sum\left[y_{S}(B) \chi^{B}(e) \mid S \in \mathcal{U}, B \in \mathcal{B}_{S}\right] \leqslant c(u),
\end{aligned}
$$

onde a primeira igualdade segue em virtude da definição (4.1); a primeira desigualdade, pois as condições $S \in \mathcal{U}, B \in \mathcal{B}_{S}$ e $u \in S$ implicam que $B \cap \rho u \neq \emptyset$; finalmente, a última desigualdade segue devido à definição (4.2). 
Como é de se esperar, o que tomará mais tempo será a prova da recíproca deste lema.

Lema 4.2 Seja $\mathbb{K}$ um sgn, $\mathcal{C}$ uma família mista, e $S \in \mathcal{C}$.

$S e B$ é uma parte $S$-arbórea de $E$, então free $B$ é $\bowtie$-fechado e $\mathcal{C} \cup\left\{\right.$ free $\left._{S} B\right\}$ é uma família mista.

Prova. Seja $B$ uma parte $S$-arbórea de $E$. Sejam $u, w \in$ free $_{S} B$ tais que $u \bowtie w$. Como $B$ evita $u$ e $w$, então $\rho_{B}(u) \cup \rho_{B}(w)=\emptyset$, donde, usando $(\rho .2)$, vem que $\rho_{B}(u \vee w) \cup \rho_{B}(u \wedge w)=\emptyset$. Segue daí que $u \vee w, u \wedge w \in$ free $_{S} B$ e, portanto, free $B$ é $\bowtie$-fechado. Agora, seja $T \in \mathcal{C}$. Tome $r \in T$ e $s \in$ free $_{S} B$ tais que $r \widetilde{凶} s$. Como $\mathcal{C}$ é uma família mista, então $r \wedge s \in S \cap T$. Vamos mostrar que $r \wedge s \in T \cap$ free $_{B} S$. Suponha, por contradição, que $r \wedge s \notin$ free $_{S} B$. Como $s \in$ free $_{S} B$, então $r \wedge s \neq s$, donde $r \wedge s \prec s$. Ora, $B$ cobre $r \wedge s$ e evita $s$ e, portanto, $B$ não é $S$-arbóreo. Esta contradição estabelece que $\mathcal{C} \cup\left\{\right.$ free $\left._{S} B\right\}$ é uma família mista.

Vamos precisar de uma operação que, informalmente, corresponde a remoção de um certo número de cópias de um subconjunto de $E$ em relação a uma parte $\bowtie$-fechada. Eis a formalização de tal ideia. Seja $\mathbb{F}$ um sgnfm, $S \in \mathcal{U}, B$ uma parte de $E$ e $\varepsilon$ um número real. Ponha

$\triangleright \quad \mu^{\varepsilon B}:=\mu\left[S \mapsto \mu S-\varepsilon\right.$, free $_{S} B \mapsto \mu\left(\right.$ free $\left.\left._{S} B\right)+\varepsilon\right]$,

e seja $\mathbb{F}^{\varepsilon B}:=\left(\mathbb{K}^{\varepsilon B}, \mu^{\varepsilon B}\right)$. Embora $\mu^{\varepsilon B}$ dependa de $S$, nunca o rotularemos com $S$ já que o contexto deixará claro o conjunto $S$ ao qual $\mu^{\varepsilon B}$ se refere. O próximo corolário estabelece condições sobre $B$ e $\varepsilon$ que garantem que $\mathbb{F}^{\varepsilon B}$ é um sgnfm.

Corolário 4.3 Seja $\mathbb{F}$ um sgnfm, $S \in \mu^{+}, B$ uma parte $S$-arbórea de $E$ e $\varepsilon$ um real tal que $0 \leqslant$ $\varepsilon \leqslant \min (\{\mu S\} \cup\{c(a) \mid a \in B\})$. Então $\mathbb{F}^{\varepsilon B}$ também é um sgnfm.

Prova. Observe que supp $\mu^{\varepsilon B}$ é uma família mista, em virtude do Lema 4.2. Além disso, a escolha de $\varepsilon$ acarreta que $\mu^{\varepsilon B} \geqslant 0, c^{\varepsilon B} \geqslant 0$. Logo, $\mathbb{F}^{\varepsilon B}$ é um sgnfm.

Seja $\mathbb{F}$ um sgnfm. É conveniente definir

$\triangleright \quad U:=U(\mathbb{F}):=\bigcup \mu^{+}$.

É evidente que para cada $u \in P$, temos que $u \in p^{+}$se e só se $u \in U$. Seja $S \in \mu^{+}$e $0 \leqslant \varepsilon \leqslant \mu S$. Por brevidade, vamos escrever $p^{\varepsilon B}:=p_{\mu^{\varepsilon B}}$. Note que para cada $u \in P$ :

$$
p^{\varepsilon B}(u)=\text { se } u \in \operatorname{cov}_{S} B \text {, então } p(u)-\varepsilon \text {, senão } p(u) .
$$

É claro que $p^{\varepsilon B}=p-\varepsilon \chi^{\operatorname{cov}_{S} B}$. Vale aqui, para $p^{\varepsilon B}$, a mesma observação que fizemos para $\mu^{\varepsilon B}$ em relação à dependência de $S$. Assim, neste caso, também não rotularemos $p^{\varepsilon B} \operatorname{com} S$. Os seguintes conjuntos serão fundamentais na discussão que segue. Definimos

$$
\begin{aligned}
& \triangleright \Gamma \mathbb{F}:=\{u \in P \mid c(\rho u)=p(u)>0\}, \mathrm{e} \\
& \triangleright \Gamma_{0} \mathbb{F}:=\{u \in P \mid c(\rho u)=p(u)\} .
\end{aligned}
$$

Finalmente, para $u, w \in P$ tais que $u \widetilde{\bowtie} w$, seja

$\triangleright \quad \mu^{+}(u, w):=\left\{S \in \mu^{+} \mid u \wedge w \in S ; u \notin S ; w \notin S\right\}$. 
O próximo lema estabelece algumas propriedades da função $p$. Em particular, o caráter supermodular de $p$ desempenhará um papel fundamental no decorrer deste capítulo; ademais, a supermodularidade de $p$ é uma pista fundamental no porquê as ideias de Lovász e Frank funcionam no contexto geral dos sgnfm.

\section{Lema 4.4 Seja $\mathbb{F} u m$ sgnfm.}

(i) Para cada $u, w \in p^{+}$:

se $u \widetilde{\bowtie} w$ então $p(u)+p(w)+\sum\left[\mu S \mid S \in \mu^{+}(u, w)\right] \leqslant p(u \vee w)+p(u \wedge w)$.

(ii) Para cada $u, w \in p^{+}$: se $u \preccurlyeq w$, então $p(u) \geqslant p(w)$.

(iii) Sejam $u, w \in p^{+}$tais que $u \widetilde{凶} w$.

Se $p(u)+p(w)=p(u \vee w)+p(u \wedge w)$, então

para cada $S \in \mu^{+}: u, w \notin S$ implica $u \vee w, u \wedge w \notin S$.

(iv) Se $\mathbb{F}$ é viável, então $\Gamma \mathbb{F} e ́ \wedge$-fechado.

Prova. Note que para cada $u \in P$ :

(*) $\quad p(u)=\sum\left[\chi^{S}(u) \mu S \mid S \in \mu^{+}\right]$.

Assim, para provar (i) é suficiente estabelecer que para todo $S \in \mu^{+}$e todo $u, w \in p^{+}$:

$(* *) \quad$ se $u \widetilde{\bowtie} w$, então $\chi^{S}(u)+\chi^{S}(w)+\chi^{\mu^{+}(u, w)}(S) \leqslant \chi^{S}(u \vee w)+\chi^{S}(u \wedge w)$.

Sejam, assim, $u, w \in p^{+}$com $u \widetilde{\bowtie} w$. Seja $S \in \mu^{+}$. A desigualdade é evidente se $u, w \notin S$, pois, neste caso, $\chi^{\mu^{+}(u, w)}(S)=\chi^{S}(u \wedge w)$. Suponha, primeiro, que $|\{u, w\} \cap S|=1$. Ajuste a notação de tal forma que $u \notin S$ e $w \in S$ (donde $\chi^{S}(w)=1$ ). Como $u \in p^{+}$, então existe $S_{u} \in \mu^{+}$tal que $u \in S_{u}$. Ora, $u \wedge w \in S \cap S_{u}$, em virtude da pim e, daí, $\chi^{S}(u \wedge w)=1$; ademais, $\chi^{\mu^{+}(u, w)}(S)=0$ pois $w, u \wedge w \in S$. Isto estabelece $(* *)$ neste caso. Suponha, agora, que $u, w \in S$. Como $S$ é $\bowtie$-fechado, então $u \vee w \in S$ e $u \wedge w \in S$, donde $\chi^{\mu^{+}(u, w)}(S)=0$, o que prova a desigualdade neste último caso.

Para a prova de (ii), sejam $u, w \in p^{+}$com $u \preccurlyeq w$. De $u \preccurlyeq w$ vem que $u \wedge w=u$. Seja $S \in \mu^{+}$ tal que $w \in S$. Como $u \in p^{+}$, então existe $S_{u} \in \mu^{+}$tal que $u \in S_{u}$. Em virtude da pim, temos que $u=u \wedge w \in S \cap S_{u}$, donde $u \in S$. Segue daí, usando $(*)$, que $p(u) \geqslant p(w)$.

Para a prova de (iii), sejam $u, w \in p^{+} \operatorname{com} u \widetilde{\bowtie} w$. Suponha que

$$
p(u)+p(w)=p(u \vee w)+p(u \wedge w) .
$$

Seja $S \in \mu^{+}$. Aliando-se esta última igualdade à $(* *)$ vem que

$$
\chi^{S}(u)+\chi^{S}(w)=\chi^{S}(u \vee w)+\chi^{S}(u \wedge w),
$$

o que estabelece (iii).

Para a prova de (iv), sejam $u, w \in \Gamma \mathbb{F} \operatorname{com} u \bowtie w$. Então

$$
p(u)+p(w)=c(u)+c(w) \geqslant c(u \vee w)+c(u \wedge w) \geqslant p(u \vee w)+p(u \wedge w) \geqslant p(u)+p(w)
$$

onde a primeira desigualdade segue do Lema 2.2; a segunda, porque $\mathbb{F}$ é viável; e a terceira, em virtude de $u, w \in p^{+}$e do item (i). Assim, vale a igualdade em todas as passagens, e daí 


$$
c(u \wedge w)=p(u \wedge w) \quad \text { e } \quad c(u \vee w)=p(u \vee w),
$$

o que estabelece $u \wedge w \in \Gamma_{0} \mathbb{F}$. Afirmamos que $u \wedge w \in p^{+}$. Suponha que não. Então

$$
p(u \vee w)=p(u)+p(w)>0 .
$$

Como $u, w \in p^{+}$, então $p(u \vee w)>p(u)$, o que contraria (ii). Concluímos, assim, que $u \wedge w \in p^{+}$. Portanto, $\Gamma \mathbb{F}$ é $\wedge$-fechado.

\section{Capacidade de um conjunto de arcos}

Vamos estender, nesta seção, a noção de capacidade de um conjunto de arcos para os sistemas generalizados de núcleos com uma família mista. Sejam $\mathbb{F}$ um sgnfm viável, $S \in \mu^{+}$, e $B$ uma parte de $c^{+}$. Seja $\varepsilon$ um número real. Lembre-se que para cada arco $a \in E$ :

$\triangleright \quad c^{\varepsilon B}(a):=$ se $a \in B$ então $c(a)-\varepsilon$ senão $c(a)$.

Além disso, para cada $u \in P$ :

$\triangleright \quad \mu^{\varepsilon B}:=\mu\left[S \mapsto \mu S-\varepsilon\right.$, free $_{S} B \mapsto \mu\left(\right.$ free $\left.\left._{S} B\right)+\varepsilon\right]$,

o que, por sua vez, implica que

$$
p^{\varepsilon B}=p-\varepsilon \chi^{\operatorname{cov}_{S} B} .
$$

Lembre-se também que $\mathbb{F}^{\varepsilon B}$ denota $\left(\mathbb{K}^{\varepsilon B}, \mu^{\varepsilon B}\right)$. Queremos encontrar um maior $\varepsilon$ tal que $\mathbb{F}^{\varepsilon B}$ é viável, ou seja, para cada $u \in P$ :

$$
c^{\varepsilon B}(\rho u) \geqslant p^{\varepsilon B}(u) .
$$

É claro que $0 \leqslant \varepsilon \leqslant \min \{c(a) \mid a \in B\}$. Seja $u$ núcleo em $P$. É claro que $u$ é relevante se $B$ cobre $u$. Assim, suponha que $u \in \operatorname{cov} B$. Admita, primeiro, que $u \in S$. Se $d_{B}(u)=1$, então $c^{\varepsilon B}(u)=$ $c(u)-\varepsilon \geqslant p(u)-\varepsilon=p^{\varepsilon B}(u)$. Se $d_{B}(u) \geqslant 2$ então

$$
p(u)-\varepsilon=p^{\varepsilon B}(u) \leqslant c^{\varepsilon B}(u)=c(u)-\varepsilon d_{B}(u)
$$

se e só se

$$
\varepsilon \leqslant \frac{c(u)-p(u)}{d_{B}(u)-1} .
$$

Finalmente, suponha que $u \notin S$. Então

$$
p(u)=p^{\varepsilon B}(u) \leqslant c^{\varepsilon B}(u)=c(u)-\varepsilon d_{B}(u)
$$

se e só se

$$
\varepsilon \leqslant \frac{c(u)-p(u)}{d_{B}(u)} .
$$

Esta discussão motiva as definições que seguem. Um núcleo $u \in P$ é especial para $B$ e $S$ se

$\triangleright \quad d_{B}(u) \geqslant 2 \quad$ ou $\quad u \in \operatorname{cov}(B) \backslash S$.

A capacidade de um núcleo $u$, especial para $B$ e $S$, é o número 


$$
\operatorname{cap}(u ; B, S):=\operatorname{cap}_{\mathbb{F}}(u ; B, S):=\frac{c(u)-p(u)}{d_{B}(u)-\chi^{S}(u)} .
$$

Observe que esta última definição implica que para cada núcleo $u$, especial para $B$ e $S$,

$$
\text { se } 0<\varepsilon \leqslant \operatorname{cap}(u ; B, S) \text {, então } c(u)-\varepsilon d_{B}(u) \geqslant p(u)-\varepsilon \chi^{S}(u)=p^{\varepsilon B}(u) .
$$

Seja $S \in \mathcal{U}$. A capacidade de $B$ em relação a $S$, denotada por $\alpha_{\mathbb{F}}(B ; S)$, ou simplesmente $\alpha(B ; S)$, é o número

$$
\min (\{c(a) \mid a \in B\} \cup\{\operatorname{cap}(u ; B, S) \mid u \in P \text { é especial para } B \text { e } S\} \cup\{\mu S\}) .
$$

Dizemos que uma parte $B$ de $S$ é uma cobertura justa de $S$ se

$\triangleright \quad B$ é uma cobertura de $S$, boa para $\check{S}$, e $\alpha(B ; S)>0$.

O lema que segue, em particular, implica que se $B$ é uma cobertura justa de $S$, então $B$ é bom para $\check{S} \cup(S \cap \Gamma)$ e $B$ evita $\bar{S} \cap \Gamma$.

Lema 4.5 Seja $\mathbb{F}$ um sgnfm viável, $S \in \mu^{+}$, e B uma parte de $c^{+}$. Então

(i) $\alpha(B ; S)>0$ se e só se $B$ é bom para $S \cap \Gamma$ e $B$ evita $\bar{S} \cap \Gamma$,

(ii) se B é $S$-arbóreo, e $0<\beta \leqslant \alpha(B ; S)$ então $\mathbb{F}^{\beta B}$ é um $\mathbf{s g n f m}$ viável, $e$

(iii) se B é uma cobertura justa de $S$, e $0<\beta \leqslant \alpha(B ; S)$, então $T \cap \Gamma \mathbb{F}^{\beta B} \supseteq T \cap \Gamma \mathbb{F}$ para cada $T \in \operatorname{supp} \mu^{\beta B}$.

Prova. Para a prova de (i), admita que $\alpha(B, S)>0$ e suponha que $B$ não é bom para $S \cap \Gamma$. Então existe $u \in \Gamma$ tal que $d_{B}(u) \geqslant 2$, donde $\operatorname{cap}(u ; B, S)=0$, o que contraria $\alpha(B ; S)>0$. Logo, $B$ é bom para $S \cap \Gamma$. Suponha, agora, que $B$ não evita $\bar{S} \cap \Gamma$. Seja $u \in \bar{S} \cap \Gamma$ tal que $B$ cobre $u$. Então $c(u)=p(u)$ e, daí, $\operatorname{cap}(u ; B, S)=0$, o que contraria $\alpha(B ; S)>0$. Suponha, agora, que $B$ é bom para $S \cap \Gamma$ e $B$ evita $\bar{S} \cap \Gamma$. É claro que, neste caso, $B$ é bom para $\Gamma$. Seja $u \in P$ tal que $u$ é especial para $B$ e $S$. Suponha que $d_{B}(u) \geqslant 2$. De $B$ bom para $\Gamma$ vem que $c(u)>p(u)$ donde cap $(u ; B, S)>0$. Suponha que $u \in \operatorname{cov}(B) \backslash S$. Como $B$ evita $\bar{S} \cap \Gamma$ e $B$ cobre $u$, temos que $u \notin \Gamma$, donde $\operatorname{cap}(u ; B, S)>0$. Por fim, $\min \{c(a) \mid a \in B\}>0$, pois $B \subseteq c^{+}$e, além disso, $\mu S>0$. Concluímos, assim, que $\alpha(B ; S)>0$, como queríamos.

Para a prova de (ii), suponha que $B$ é $S$-arbóreo e $0<\beta \leqslant \alpha(B ; S)$. Como $B$ é $S$-arbóreo e $\beta \leqslant \alpha(B ; S)$ vem, pelo Corolário 4.3 , que $\mathbb{F}^{\beta B}$ é um sgnfm. Falta verificar que $\mathbb{F}^{\beta B}$ é viável. Tome $u \in P$. Se $u \notin \operatorname{cov} B$ então

$$
c^{\beta B}(u)=c(u) \geqslant p(u)=p^{\beta B}(u)
$$

Suponha, pois, que $u \in \operatorname{cov} B$. Se $u$ é especial para $B$ e $S$, então

$$
c^{\beta B}(u)=c(u)-\beta d_{B}(u) \geqslant p(u)-\beta \chi^{S}(u)=p^{\beta B} .
$$

Suponha que $u$ não é especial para $B$ e $S$. Então $d_{B}(u)=1$. Como $u \in \operatorname{cov} B$, então $u \in S$. Daí

$$
c^{\beta B}(u)=c(u)-\beta \geqslant p(u)-\beta=p^{\beta B}(u) .
$$

Logo, $\mathbb{F}^{\beta B}$ é viável. 
Para a prova de (iii), suponha que $B$ é uma cobertura justa de $S$, e $0<\beta \leqslant \alpha(B ; S)$. Seja $T$ um elemento de supp $\mu^{\beta B}$ e tome $u \in T \cap \Gamma \mathbb{F}$. Então $c(u)=p(u)$. Como $\alpha(B ; S)>0$, então, por (i), $d_{B}(u) \leqslant \chi^{S}(u)$ e, assim, $c^{\beta B}(u)=p^{\beta B}(u)$. Entretanto, $u \in T$ e $T \in \operatorname{supp} \mu^{\beta B}$ implicam $p^{\beta B}(u)>0$ e, portanto, $u \in T \cap \Gamma \mathbb{F}^{\beta B}$, como queríamos.

\subsection{Alguns teoremas sobre empacotamentos}

Podemos, agora, provar alguns teoremas sobre empacotamentos em sgnfms. Seja $\mathbb{K}$ um sgn, $B$ uma parte de $c^{+}, S$ uma parte $\bowtie$-fechada de $P, R$ uma parte de $P$ e $\varepsilon$ um número real positivo. Lembre-se que $(R, S, B, \varepsilon)$ é evitável se

(n.0) $r \in R, s \in S$ e $r \widetilde{凶} s$ implicam $r \wedge s \in S$,

(n.1) $R$ é $\wedge$-fechado,

(n.2) $R \cap$ free $_{S} B=\emptyset$,

(n.3) para cada $r \in R, s \in S \operatorname{com} r \widetilde{凶} s:$

free $_{S} B \ni r \wedge s \prec r$ implica $c^{\varepsilon B}(r \wedge s)>c^{\varepsilon B}(r)$, e

(n.4) para cada $r, s \in R$ :

$r \bowtie s$ implica $c^{\varepsilon B}(r)+c^{\varepsilon B}(s)=c^{\varepsilon B}(r \vee s)+c^{\varepsilon B}(r \wedge s)$.

Lema 4.6 Seja $\mathbb{F}$ um sgnfm viável, $S \in \mu^{+}$, e B uma parte $S$-arbórea de c $c^{+} \operatorname{com} 0<\alpha(B ; S)=: \varepsilon$. Seja $R:=\Gamma \mathbb{F}^{\varepsilon B} \backslash$ free $_{S} B$. Então

(i) $(R, S, B, \varepsilon)$ é evitável,

(ii) se a $\in c^{+}$evita $R$ e $B \cup\{a\}$ é S-arbóreo, então $\beta>0$ e $\mathbb{F}^{\beta(B \cup\{a\})}$ é um $\mathbf{s g n f m}$ viável, onde $\beta:=\alpha(B \cup\{a\} ; S), e$

(iii) se $\mathbb{F}$ é inteiro e $a \in c^{+}$evita $R$ e $B \cup\{a\}$ é S-arbóreo, então $\varepsilon \geqslant 1$ implica $\beta \geqslant 1$, onde $\beta:=\alpha(B \cup\{a\} ; S)$.

Prova. Observe, primeiro, que o Lema 4.5(ii) implica que $\mathbb{F}^{\varepsilon B}$ é um sgnfm viável. Eis a prova de (i). Sejam $r \in R$ e $s \in S$ tais que $r \widetilde{凶} s$. Como $r \in \Gamma \mathbb{F}^{\varepsilon B}$, então $p(r)>0$, donde $r \in S^{\prime}$ para algum $S^{\prime} \in \mu^{+}$. Segue daí, pela pim, que $r \wedge s \in S$. Isto verifica (n.0).

Seja $r, s \in R \operatorname{com} r \bowtie s$. Então $r, s \in \Gamma \mathbb{F}^{\varepsilon B}$ e, portanto,

$$
c^{\varepsilon B}(u)=p^{\varepsilon B}(r)>0 \quad \text { e } \quad c^{\varepsilon B}(s)=p^{\varepsilon B}(s)>0 .
$$

Assim, o Lema 4.4 implica que

$$
\begin{aligned}
& p^{\varepsilon B}(r)+p^{\varepsilon B}(s)=c^{\varepsilon B}(r)+c^{\varepsilon B}(s) \geqslant c^{\varepsilon B}(r \vee s)+c^{\varepsilon B}(r \wedge s) \\
& \geqslant p^{\varepsilon B}(r \vee s)+p^{\varepsilon B}(r \wedge s) \geqslant p^{\varepsilon B}(r)+p^{\varepsilon B}(s),
\end{aligned}
$$

donde vale a igualdade em todas as passagens e, portanto,

$$
c^{\varepsilon B}(r)+c^{\varepsilon B}(s)=c^{\varepsilon B}(r \vee s)+c^{\varepsilon B}(r \wedge s),
$$

o que prova (n.4). Além disso, a viabilidade de $\mathbb{F}^{\varepsilon B}$ implica, de acordo com o Lema 4.4iv, que $\Gamma \mathbb{F}^{\varepsilon B}$ é $\wedge$-fechado e, portanto, $r \wedge s \in \Gamma \mathbb{F}^{\varepsilon B}$. No entanto, as condições $r \bowtie s$, e $r, s \notin$ free $B$, e 
$p^{\varepsilon B}(r)+p^{\varepsilon B}(s)=p^{\varepsilon B}(r \vee s)+p^{\varepsilon B}(r \wedge s)$, aliadas ao Lema 4.4iii, implicam que $r \wedge s \notin$ free $B$ e, portanto, $r \wedge s \in R$, o que prova $(n .1)$.

A propriedade (n.2) é óbvia. Para a prova de (n.3), sejam $r \in R$ e $s \in S$ tais que $r \widetilde{\bowtie} s$. Suponha, ademais, que free $B \ni r \wedge s \prec r$. Note que $p^{\varepsilon B}(r)>0$ uma vez que $r \in R$ e, além disso, $p^{\varepsilon B}(r \wedge s)>$ 0 , pois $r \wedge s \in$ free $_{S} B$ e $\varepsilon>0$. Segue daí, pelo Lema 4.4ii, que $p^{\varepsilon B}(r \wedge s) \geqslant p^{\varepsilon B}(r)$. No entanto, $r \wedge s \in$ free $_{S} B$ e $r \notin$ free $_{S} B$ acarretam $p^{\varepsilon B}(r \wedge s)>p^{\varepsilon B}(r)$. Agora,

$$
c^{\varepsilon B}(r \wedge s) \geqslant p^{\varepsilon B}(r \wedge s)>p^{\varepsilon B}(r)=c^{\varepsilon B}(r),
$$

onde a primeira desigualdade segue da viabilidade de $\mathbb{F}^{\varepsilon B}$, e a igualdade vem de $r \in \Gamma \mathbb{F}^{\varepsilon B}$. Isto completa a prova de (i).

Vamos, agora, lidar com as provas de (ii) e (iii). Para isso, seja $a \in c^{+}$tal que $a$ evita $R$ e suponha que $B^{\prime}:=B \cup\{a\}$ é $S$-arbóreo. Observe que se $a \in B$, então não há nada a provar. Suponha, no que segue, que $a \notin B$.

Se $\beta=\varepsilon$ ou $\beta=c(a)$, então $\beta>0$ e não há mais nada a provar. Suponha, no que segue, que $\beta<\min \{\varepsilon, c(a)\}$. Então $\beta=\operatorname{cap}(u ; B \cup\{a\}, S)$ para algum $u \in P$, especial para $B \cup\{a\}$ e $S$, tal que $a$ cobre $u$. Daí $d_{B \cup\{a\}}(u) \geqslant 2$ ou $u \in \operatorname{cov}(B \cup\{a\}) \backslash S$. Em ambos os casos, $u \notin$ free $B$ e $a$ evita $R$ implicam que $u \notin \Gamma \mathbb{F}^{\varepsilon B}$. Assim, $c^{\varepsilon B}(u)>p^{\varepsilon B}(u)$ e, consequentemente, $c(u)>p(u)$ o que, por sua vez, fornece $\operatorname{cap}(u ; B \cup\{a\}, S)>0$. Portanto, $\beta>0$. Logo, o Lema 4.5(ii) implica que $\mathbb{F}^{\beta B^{\prime}}$ é um sgnfm viável. Isto completa a prova de (ii).

Para a prova de (iii), note que $c \geqslant 1$. Assim, é suficiente mostrar que $\rightsquigarrow \quad$ para cada $z \in P$ : se $z$ é especial para $B^{\prime}$ e $S$, então $\operatorname{cap}\left(z ; B^{\prime}, S\right) \geqslant 1$.

Seja, pois, $z \in P$ especial para $B^{\prime}$ e $S$. Se $a$ evita $z$, então $d_{B}(z)=d_{B^{\prime}}(z)$ e, consequentemente, $\operatorname{cap}\left(z ; B^{\prime}, S\right)=\operatorname{cap}(z ; B, S) \geqslant 1$. No que segue, vamos supor que $a$ cobre $z$. O arco $a$ evita $R$ mas cobre $z$, donde $z \notin R$. Afirmamos que $c^{\varepsilon B}(\rho z)>p^{\varepsilon B}(z)$. De fato, suponha, do contrário, que $c^{\varepsilon B}(z)=p^{\varepsilon B}(z)$, ou seja, $z \in \Gamma \mathbb{F}^{\varepsilon B}$. Se $d_{B^{\prime}}(z) \geqslant 2$, então $d_{B}(z) \geqslant 1$, donde $z \notin$ free ${ }_{S} B$ e, portanto, $z \in R$, o que é uma contradição. Se, por outro lado, $z \in \operatorname{cov}\left(B^{\prime}\right) \backslash S$, então $z \notin$ free $_{S} B$ e, portanto, $z \in R$, o que, novamente, é uma contradição. Concluímos, assim, que $c^{\varepsilon B}(z)>p^{\varepsilon B}(z)$. Segue daí que

$$
c(z)-\varepsilon d_{B}(z)=c^{\varepsilon B}(z)>p^{\varepsilon B}(z)=p(z)-\varepsilon \chi^{S}(z) .
$$

Suponha, primeiro, que $d_{B^{\prime}}(z) \geqslant 2$. Então

$$
c(z)-p(z)>\varepsilon\left(d_{B}(z)-\chi^{S}(z)\right)>d_{B}(z)-\chi^{S}(z) .
$$

Ora, $c$ e $p$ são inteiros, donde $c(z)-p(z) \geqslant d_{B}(z)-\chi^{S}(z)+1$. Segue, assim, que

$$
\operatorname{cap}\left(z ; B^{\prime}, S\right)=\frac{c(z)-p(z)}{d_{B^{\prime}}(z)-\chi^{S}(z)} \geqslant 1,
$$

como queríamos. Suponha, finalmente, que $d_{B^{\prime}}(z)=1$. Então $c(z)=c^{\varepsilon B}(z)>p^{\varepsilon B}(z)=p(z)$ e, portanto, de $c$ e $p$ inteiros vem que $\operatorname{cap}\left(z ; B^{\prime}, S\right)=c(z)-p(z) \geqslant 1$. Isto estabelece (iii) e completa a prova do lema. 
Os dois próximos teoremas garantem a existência de coberturas justas em sistemas generalizados de núcleos com uma família mista. As provas são similares, por isso, só provamos o segundo deles.

Teorema 4.7 Seja $\mathbb{F}$ sgnfm viável e $S \in \mu^{+}$.

Então existe uma cobertura justa $B$ de $S$ tal que $\mathbb{F}^{\alpha(B ; S) B}$ é um $\mathbf{s g n f m}$ viável.

Teorema 4.8 Seja $\mathbb{F}$ sgnfm viável e inteiro e $S \in \mu^{+}$.

Então existe uma cobertura justa $B$ de $S$ tal que $\mathbb{F}^{B}$ é um $\mathbf{s g n f m}$ viável e inteiro.

Prova. Seja $B$ uma parte maximal de $c^{+}$dotada das seguintes propriedades:

$B$ é $S$-arbórea, $B$ é boa para $\check{S}$, e $\alpha(B ; S) \geqslant 1$.

O Lema 4.5ii implica que $\mathbb{F}^{B}$ é um sgnfm viável; mais ainda, $\mathbb{F}^{B}$ é inteiro. Se $B$ cobre $S$, então $B$ é uma cobertura justa de $S$. Suponha, então, que $B$ não cobre $S$. Seja $R:=\Gamma \mathbb{F}^{B} \backslash$ free $_{S} B$. O Lema 4.6i implica que $(R, S, B, 1)$ é evitável e, daí, pela Proposição 2.6, existe um arco $a \in c^{+}$ que evita $R$ e tal que $B \cup\{a\}$ é $S$-arbóreo, bom para $\check{S}$, e $\operatorname{cov}(B \cup\{a\}) \supset \operatorname{cov} B$. Por brevidade, escreva $B^{\prime}:=B \cup\{a\}$ e $\beta:=\alpha\left(B^{\prime} ; S\right)$. Ora, o Lema 4.6ii implica que $\mathbb{F}^{\beta B^{\prime}}$ é um sgnfm viável, e o Lema 4.6iii implica que $\beta \geqslant 1$. Além disso, $\beta \geqslant 1$ e o Lema 4.5ii acarretam que $\mathbb{F}^{B^{\prime}}$ é um sgnfm viável que é, claramente, inteiro. Assim, $B^{\prime}$ contraria a escolha de $B$. Esta contradição mostra que $B$ cobre $S$, como queríamos.

Observe que o teorema precedente poderia ser enunciado da seguinte forma alternativa que será útil nos teoremas envolvendo empacotamentos de coberturas que exibiremos posteriormente.

Teorema 4.9 Seja $\mathbb{F}$ um sgnfm viável e inteiro, e $S \in \mu^{+}$. Seja B uma parte $S$-arbórea de $c^{+}$, boa para $\check{S}$, e tal que $\mathbb{F}^{B}$ é um $\mathbf{s g n f m}$ viável e inteiro. Então existe uma cobertura $B^{\prime}$ de $S$, boa para $\check{S}$, tal que $B^{\prime} \supseteq B$ e $\mathbb{F}^{B^{\prime}}$ é um sgnfm viável e inteiro.

Para resumir a discussão vamos exibir o algoritmo que está implícito na prova do Teorema 4.8 que recebe um sgnfm viável $\mathbb{F}$ e $S \in \mu^{+}$e devolve uma cobertura justa de $S$.

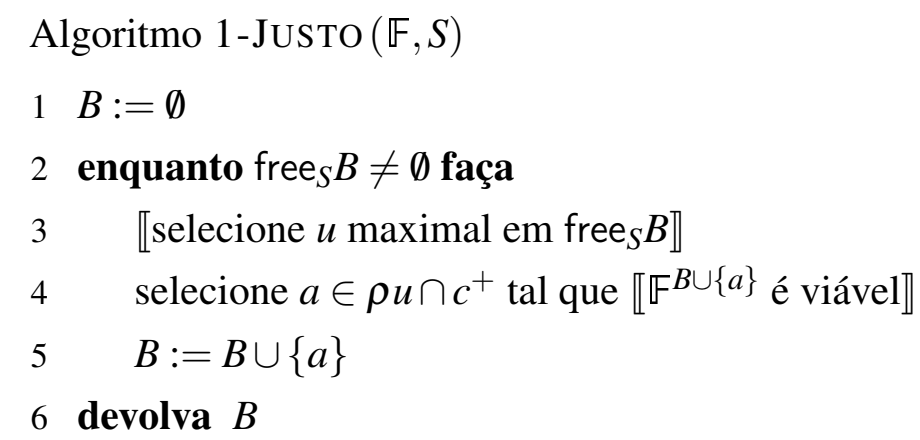

O seguinte teorema, que constitui uma solução para o Problema 1, segue por indução em $\Sigma[|S| \mu(S) \mid S \in \mu]$, usando-se o Teorema 4.8. Observe que a prova sugere um algoritmo, exibido no que segue, que em geral não é polinomial em $|E|$, a menos que estejamos lidando com a versão não-capacitada. Forneceremos um tal algoritmo, de complexidade polinomial, no Capítulo 5. 
Teorema 4.10 Se $\mathbb{F}$ é um $\mathbf{s g n f m}$ viável e inteiro, então $\mathbb{F}$ empacota.

Este teorema, por sua vez, generaliza a versão forte do Teorema de Edmonds; também generaliza um teorema de Szegő [Sze01] e um outro de Bércsi e Frank [BF08].
Algoritmo EMPACOTA (F)
1 para todo $S \in \mathcal{U}$ faça
$2 y_{S}:=\overrightarrow{0}$
3 enquanto $\mu^{+} \neq \emptyset$ faça
$4 \quad$ selecione $S \in \mu^{+}$
$5 \quad B:=1-\operatorname{JUSTO}(\mathbb{F}, S)$
$6 \quad y_{S}:=y_{S}\left[B \mapsto y_{S}(B)+1\right]$
$7 \quad \mathbb{F}:=\mathbb{F}^{B}$
8 devolva $\emptyset\left[\mathcal{U} \ni S \mapsto y_{S}\right]$ 


\section{Capítulo 5}

\section{Empacotamentos em sgnfm}

Neste capítulo, vamos provar teoremas sobre empacotamentos inteiros e fracionários em sistemas generalizados de núcleos com uma família mista. Ambos os teoremas fornecerão melhores limitantes que os estabelecidos por Gabow e Manu [GM98], e Schrijver [Sch03], quando especializados tanto para o problema do empacotamento de arborescências geradoras quanto para o problema do empacotamento de ramificações geradoras.

\subsection{Dois teoremas sobre empacotamentos}

Vamos, nesta seção, provar dois teoremas: um sobre empacotamento de sgnfms, e, o outro, sobre empacotamentos de sgn-c-mfs: um $\mathbf{s g n f m}(\mathbb{K}, \boldsymbol{\mu})$ é um sgn-c-fm se $\mathbb{K}$ é um sgn-c. Os teoremas sugerem um algoritmo que produz tais empacotamentos cuja complexidade é polinomial. Curiosamente, este algoritmo é o mesmo em ambos os teoremas e, por isso, as provas serão feitas em conjunto. A diferença é que a natureza mais restrita dos sgn-c-fms - especificamente, a possibilidade de realizar um processo de descruzamento, como aquele especificado no Capítulo 3 - permitirá produzir, diante de certas condições, um melhor limitante para o tamanho de um empacotamento.

Uma das ideias envolvidas na prova do teorema sobre empacotamentos consiste em modificar o sgnfm de tal forma que o posto de um certo conjunto de vetores aumente. Para isso, o seguinte lema, que segue por um argumento básico de álgebra linear, será útil.

Lema 5.1 Seja $M$ um conjunto finito e $Q$ o poliedro $\left\{x \in \mathbb{R}^{n} \mid a_{i} x \geqslant b_{i}\right.$ para cada $\left.i \in M\right\}$, onde $a_{i} \in \mathbb{R}^{n}$ e $b_{i} \in \mathbb{R}$ para cada $i \in M$. Seja, também, $I:=\left\{i \in M \mid \forall x \in Q: a_{i} x=b_{i}\right\}$ e $A_{I}:=\left\{a_{i} \mid i \in I\right\}$. Se existem $d \in \mathbb{R}^{n}, \delta \in \mathbb{R}$ e $x, z \in Q$ tais que $d x \leqslant \delta$ e $d z>\delta$, então $d \notin \operatorname{span} A_{I}$.

Prova. Sejam $d \in \mathbb{R}^{n}, \delta \in \mathbb{R}$ e $x, z \in Q$ tais que $d x \leqslant \delta$ e $d z>\delta$. Suponha, por contradição, que $d \in \operatorname{span} A_{I}$. Então existe $y$ tal que $y A_{I}=d$. Segue daí que

$$
\delta \geqslant d x=\left(y A_{I}\right) x=y\left(A_{I} x\right)=y b_{I}=y\left(A_{I} z\right)=\left(y A_{I}\right) z=d z>\delta,
$$

o que é uma contradição. Logo, $d \notin \operatorname{span} A_{I}$. 
Um prenúncio da prova Antes de entrarmos em detalhes mais técnicos, é conveniente tecer alguns comentários sobre a técnica de prova que vamos utilizar, sobretudo naquela que envolve os sgn-c-fms. Suponha que $\mathbb{F}$ é um sgn-c-fm viável e inteiro. O tamanho do empacotamento que produziremos dependerá de $\left|c^{+}\right|+\left|\mu^{+}\right|+\zeta$, onde $\zeta$ é um parâmetro que gostaríamos que fosse não maior (assintoticamente) que $\left|c^{+}\right|$. A ideia da prova consiste em tomar uma cobertura justa $B$ de $S$, e tentar remover um número grande, digamos $k$, de cópias de $B$ de $\mathbb{F}$. Se $k=\mu S$ ou $k=c(a)$ para algum arco $a$ de $B$, então indutivamente podemos encontrar um empacotamento de $\mathbb{F}^{k B}$ que pode ser estendido para um de $\mathbb{F}$ adicionando-se $k$ cópias de $B$. Quando $k<\mu S$ e $k<c(a)$ para todo arco $a$ de $B$, então temos que, de alguma forma, tentar diminuir $\zeta$. Para isso, vamos considerar o conjunto dos vetores $W:=\left\{\chi^{\rho u} \mid u \in \Gamma_{0} \mathbb{F}\right\}$ e o sgn-c-fm $\mathbb{F}^{k B}$. Não é difícil se convencer de que $\mathbb{F}^{(k+1) B}$ é inviável. Assim, vamos tomar uma parte maximal $A$ de $B$ tal que $\left(\mathbb{F}^{k B}\right)^{A}$ é viável. A maximalidade de $A$ garante que existe um arco $e \in B$ tal que $\tilde{F}:=\left(\mathbb{F}^{k B}\right)^{A \cup\{e\}}$ é inviável. Vamos, então, escolher um núcleo $w$ tal que $\tilde{c}(w)=\tilde{p}(w)-1$ e mostrar que o posto do conjunto $W \cup\left\{\chi^{\rho w}\right\}$ é maior que o posto de $W$. Para poder produzir um empacotamento ainda melhor, vamos ter que utilizar a operação de descruzamento exibida no Capítulo 3. Lembre-se que se $L$ é uma parte laminar de $P$ e $t$ é um núcleo de $P$, então existe uma $t$-spi $\mathbf{f} \subseteq L_{t}$ tal que $L \cup\{t \vee \mathbf{f}\}$ é laminar. Vamos mostrar que, diante destas condições, podemos escolher $t$ de tal forma que se $L$ é uma parte laminar, de tamanho máximo, de $\Gamma_{0} \mathbb{F}$ então $t \vee \mathbf{f} \notin L$ e, ademais, $L \cup\{t \vee \mathbf{f}\}$ é um subconjunto de $\Gamma_{0} \mathbb{F}^{\prime \prime}$, onde $\mathbb{F}^{\prime \prime}$ é um sgn-c-fm obtido de $\mathbb{F}$ pela remoção de certas coberturas justas. Esta última operação é tecnicamente sutil pois o argumento tem que levar em conta a supermodularidade de $p$. No entanto, estamos tomando uma parte $L$ de $\Gamma_{0} \mathbb{F}$ e $p$ é supermodular em $\Gamma \mathbb{F}$. Há duas alternativas para contornar esta limitação. A primeira delas consiste em considerar os elementos maximais de $\mu^{+}$, aumentando-se sua multiplicidade de uma unidade, e modificar $\mathbb{F}$ adicionando-se um novo arco, de capacidade 1 , para cada elemento da dupla $(u, S)$, onde $u \in S$ e $S \in \mu^{+}$. Suponha que $\mathbb{G}$ é este novo sgn-c-fm. Desta forma, nos teoremas, teríamos que lidar tanto com $\mathbb{F}$ quanto com $\mathbb{G}$, fazendo um malabarismo entre ambos para garantir a correção dos argumentos. Embora tal ideia pareça intuitivamente mais clara, ela esconde uma operação fundamental que num certo sentido preserva a supermodularidade de $p$. Optamos, desta forma, pela segunda alternativa, que passamos a descrever.

Seja $\mathbb{F}$ um sgnfm e $V$ uma parte de $P$. Dizemos que $p$ é legal para $V$ em $\mathbb{F}$ se

(i) $V \supseteq U \mathbb{F}$,

(ii) para todo $S \in \mu^{+}$: se $s \in S$ e $v \in V$ e $s \widetilde{\bowtie} v$, então $s \wedge v \in S$.

(iii) para todo $u, w \in V$ tal que $u \bowtie w$ :

$$
p(u)+p(w)+\sum\left[\mu(S) \mid S \in \mu^{+}(u, w)\right] \leqslant p(u \wedge w)+p(u \vee w) .
$$

Lembre-se que $\mu^{+}(u, w)$ denota o conjunto $\left\{S \in \mu^{+} \mid u \wedge w \in S ; u \notin S ; w \notin S\right\}$.

Lema 5.2 Se $\mathbb{F}$ é um sgn-c-fm, então pé legal para UF em $\mathbb{F}$.

Prova. Seja $\mathbb{F}$ um sgn-c-fm. A propriedade (5.1i) é evidente; (5.1ii) é válida uma vez que $\mu^{+}$é uma família mista e, portanto, $U \mathbb{F}$ é $\wedge$-fechado. Finalmente, o Lema 4.4i implica (5.1iii). 
Lema 5.3 Seja $\mathbb{F}$ um sgnfm, $V$ uma parte de $P, S \in \mu^{+}, B$ uma parte $S$-arbórea de $E$, e $k \in \mathbb{R}$. Se p é legal para $V$ em $\mathbb{F} e 0 \leqslant k \leqslant \mu S$, então $p^{k B}$ é legal para $V$ em $\mathbb{F}^{k B}$.

Prova. Seja $S^{\circ}:=$ free $_{S} B$ e $S^{\bullet}:=\operatorname{cov}_{S} B$. Suponha que $p$ é legal para $V$ em $\mathbb{F}$ e $0 \leqslant k \leqslant \mu S$. Vamos mostrar que $p^{k B}$ é legal para $V$ em $\mathbb{F}^{k B}$. É claro que $V \supseteq U \mathbb{F}^{k B}$ e, portanto, vale (5.1i). Para a prova de (5.1ii), sejam $T \in\left(\mu^{k B}\right)^{+}, t \in T$ e $v \in V$ tais que $t \widetilde{\bowtie} v$. Se $T \in \mu^{+}$, então $t \wedge v \in T$. Suponha que $T \notin \mu^{+}$. Então $T=S^{\circ}$. Como $S^{\circ} \subseteq S$ e $S \in \mu^{+}$, então $t \wedge v \in S$. Ora, $B$ é $S$-arbóreo e $t \in S^{\circ}$, donde $t \wedge v \in S^{\circ}$, estabelecendo (5.1ii). Resta verificar (5.1iii). Sejam $u, w \in V$ tais que $u \bowtie w$.

Fato 5.3.1 $p^{k B}(u)+p^{k B}(w)+\sum\left[\mu^{k B}(T) \mid T \in\left(\mu^{k B}\right)^{+}(u, w)\right] \leqslant p^{k B}(u \wedge w)+p^{k B}(u \vee w)$.

- Vamos precisar da seguinte definição: para cada $X \subseteq P$, pomos

$\triangleright \quad \delta^{u w}(X):=$ se $\{u, w\} \cap X=\emptyset$ e $u \wedge w \in X$ então 1 , senão 0.

Vamos mostrar que

$(*) \quad \chi^{S^{\bullet}}(u)+\chi^{S^{\bullet}}(w)+\delta^{u w}(S) \geqslant \chi^{S^{\bullet}}(u \vee w)+\chi^{S^{\bullet}}(u \wedge w)+\delta^{u w}\left(S^{\circ}\right)$.

A prova é dividida em uma série de casos:

Caso $1 u, w \notin S$.

Então $\chi^{S^{\bullet}}(u)=\chi^{S^{\bullet}}(w)=0$. Ora, devido a (5.1ii), $u \vee w \notin S$, donde $\chi^{S^{\bullet}}(u \vee w)=0$.

Suponha que $\chi^{S^{\bullet}}(u \wedge w)=0$. Então $\delta^{u w}(S)=\delta^{u w}\left(S^{\circ}\right)$. Logo, $(*)$ é válida.

Suponha que $\chi^{S^{\bullet}}(u \wedge w)=1$. Então $u \wedge w \in S$, donde $\delta^{u w}(S)=1$. Além disso, $u \wedge w \notin S^{\circ} \mathrm{e}$ daí $\delta^{u w}\left(S^{\circ}\right)=0$. Logo, $(*)$ é válida.

\section{Caso $2 u, w \in S$.}

Suponha, primeiro, que $\chi^{S^{\bullet}}(u)=\chi^{S^{\bullet}}(w)=1$. Então $\delta^{u w}(S)=0$. Como $B$ é $S$-arbóreo e $u \vee w \in S$, então $\chi^{S^{\bullet}}(u \vee w)=1$. Note que

$$
\chi^{S^{\bullet}}(u \wedge w)=1 \Rightarrow \delta^{u w}\left(S^{\circ}\right)=0 \quad \text { e } \quad \chi^{S^{\bullet}}(u \wedge w)=0 \Rightarrow \delta^{u w}\left(S^{\circ}\right)=1 .
$$

Segue daí que $\chi^{S^{\bullet}}(u \wedge w)+\delta^{u w}\left(S^{\circ}\right)=1$, o que estabelece $(*)$ neste caso.

Suponha, agora, que $\chi^{S^{\bullet}}(u)=\chi^{S^{\bullet}}(w)=0$. Então $\delta^{u w}\left(S^{\circ}\right)=\delta^{u w}(S)=0$. Ora, como $B$ é $S$-arbóreo e $u \wedge w \in S$, então $\chi^{S^{\bullet}}(u \wedge w)=0$. Agora, vamos mostrar que $\chi^{S^{\bullet}}(u \vee w)=0$, o que prova $(*)$, neste caso. De fato, suponha, por contradição, que $\chi^{S^{\bullet}}(u \vee w)=1$ e seja $a \in \rho_{B}(u \vee w)$. Agora, $(\rho .2)$ implica que $a \in \rho u \cup \rho w$, donde $\chi^{S^{\bullet}}(u)+\chi^{S^{\bullet}}(w) \geqslant 1$, o que é uma contradição.

Suponha, enfim, que $\chi^{S^{\bullet}}(u)+\chi^{S^{\bullet}}(w)=1$. Ajuste a notação de tal forma que $\chi^{S^{\bullet}}(u)=1$. Como $B$ é $S$-arbóreo, $u \wedge w \in S$, e $\chi^{S^{\bullet}}(w)=0$, então $\chi^{S^{\bullet}}(u \wedge w)=0$. No entanto, $w \in S^{\circ}$ implica que $\delta^{u w}\left(S^{\circ}\right)=0$, o que estabelece $(*)$. 
Caso $3|\{u, w\} \cap S|=1$.

Ajuste a notação de tal forma que $u \in S$ e $w \notin S$; neste caso $\chi^{S^{\bullet}}(w)=0$. Note que $u \wedge w \in S$ e $\delta^{u w}(S)=0$. Se $u \vee w \in S$, então (5.1ii) implica que $w \in S$, o que é uma contradição. Assim, $u \vee w \notin S$ e, portanto, $\chi^{S^{\bullet}}(u \vee w)=0$.

Suponha que $\chi^{S^{\bullet}}(u)=1$. Se $\chi^{S^{\bullet}}(u \wedge w)=1$, então $\delta^{u w}\left(S^{\circ}\right)=0$, o que estabelece $(*)$. Se $\chi^{S^{\bullet}}(u \wedge w)=0$, então $(*)$ decorre de $\delta^{u w}\left(S^{\circ}\right) \leqslant 1$.

Suponha que $\chi^{S^{\bullet}}(u)=0$. Então $\delta^{u w}\left(S^{\circ}\right)=0$. Afirmamos que $\chi^{S^{\bullet}}(u \wedge w)=0$, o que prova (*). De fato, suponha que $\chi^{S^{\bullet}}(u \wedge w)=1$. Seja $a \in \rho_{B}(u \wedge w)$. Então $(\rho .3)$ implica que $a \in \rho u \cup \rho w$, donde $\chi^{S^{\bullet}}(u)+\chi^{S^{\bullet}}(w)=1$, o que é uma contradição.

Agora, observe que $p^{k B}(v)=p(v)-k \chi^{S^{\bullet}}(v)$ para cada $v \in V$ e, ademais,

$$
\sum\left[\mu^{k B}(T) \mid T \in\left(\mu^{k B}\right)^{+}(u, w)\right]=\sum\left[\mu T \mid T \in \mu^{+}(u, w)\right]-k \delta^{u w}(S)+k \delta^{u w}\left(S^{\circ}\right),
$$

donde, em virtude de $(*)$, vem que

$$
p^{k B}(u)+p^{k B}(w)+\sum\left[\mu^{k B}(T) \mid T \in\left(\mu^{k B}\right)^{+}(u, w)\right] \leqslant p^{k B}(u \wedge w)+p^{k B}(u \vee w),
$$

o que completa a prova do fato.

Segue daí que $p^{k B}$ é legal para $V$ em $\mathbb{F}^{k B}$, como queríamos.

Seja $\mathbb{F}$ um sgnfm. Lembre-se que para uma parte $X$ de $P$, denotamos por rank $(X)$ a cardinalidade de um conjunto linearmente independente de tamanho máximo de $\left\{\chi^{\rho u} \mid u \in X\right\}$. Uma conveção similar é feita a respeito do conjunto span $X$. É conveniente definir

$\triangleright \quad M \mathbb{F}:=\check{P} \cap\left(\cap \mu^{+}\right)$.

Em palavras, $M \mathbb{F}$ é o conjunto dos núcleos minimais de $P$ que participam de todos os elementos de $\mu^{+}$.

Vamos, agora, enunciar o teorema sobre empacotamento fracionário. No entanto, omitimos sua prova uma vez que a mesma é uma versão mais simples da prova do teorema sobre empacotamento inteiro, para o qual uma prova detalhada é fornecida.

Teorema 5.4 Seja $\mathbb{F}$ um $\mathbf{s g n f m}$ viável.

Então $\mathbb{F}$ possui um empacotamento y tal que $\left|y^{+}\right| \leqslant\left|c^{+}\right|-\operatorname{rank}\left(M \mathbb{F} \cup \Gamma_{0} \mathbb{F}\right)+\left|\mu^{+}\right|$.

Eis, então, o primeiro teorema sobre empacotamentos inteiros. Na verdade, no que segue, há dois teoremas: um envolvendo sgnfms e, o outro, o arcabouço mais restrito dos sgn-c-fms. Antes, porém, vamos precisar da definição que segue. Para uma parte $X$ de $P, \gamma X$ é a cardinalidade de um subconjunto laminar de $X$ de cardinalidade máxima.

Teorema 5.5 Seja $\mathbb{F}$ um sgnfm viável e inteiro. Então

(i) $\mathbb{F}$ possui um empacotamento inteiro y tal que $\left|y^{+}\right| \leqslant 2\left(\left|c^{+}\right|-\operatorname{rank}\left(M \mathbb{F} \cup \Gamma_{0} \mathbb{F}\right)\right)+\left|\mu^{+}\right|$.

(ii) se $\mathbb{F}$ é um sgn-c-fm, então $\mathbb{F}$ possui um empacotamento inteiro y tal que 


$$
\left|y^{+}\right| \leqslant\left|c^{+}\right|+\left|\mu^{+}\right|+\gamma(U \mathbb{F} \backslash \check{P})-\gamma(\Gamma \mathbb{F} \backslash \check{P})-\operatorname{rank}\left(M \mathbb{F} \cup \Gamma_{0} \mathbb{F}\right) .
$$

Prova. Vamos provar (i) e a seguinte afirmação:

(ii)' Seja $V$ uma parte de $P$. Se $\mathbb{F}$ é um sgn-c-fm e $p$ é legal para $V$, então $\mathbb{F}$ possui um empacotamento inteiro $y$ tal que

$$
\left|y^{+}\right| \leqslant\left|c^{+}\right|+\left|\mu^{+}\right|+\gamma(V \backslash \check{P})-\gamma\left(\left(V \cap \Gamma_{0} \mathbb{F}\right) \backslash \check{P}\right)-\operatorname{rank}\left(M \mathbb{F} \cup \Gamma_{0} \mathbb{F}\right) .
$$

As provas dos itens (i) e (ii)' contêm diversos passos em comum. Na maior parte do tempo os argumentos que vamos utilizar se aplicam a ambos os casos. Assim, para frisar que estamos lidando com o caso (ii)', que requer argumentos específicos, vamos usar uma outra fonte e, ademais, envolver o argumento com os delimitadores " $\checkmark$ " e “ $\triangleleft$ ". Além disso, sempre que usarmos esta outra fonte, vamos admitir, sobretudo para evitar o excesso de repetições, que $\mathbb{F}$ é um sgn-c-fm e que $V$ é uma parte de $P$ tal que $p$ é legal para $V$. Vamos escrever, para abreviar,

(i) $\varphi \mathbb{F}:=2\left(\left|c^{+}\right|-\operatorname{rank}\left(M \mathbb{F} \cup \Gamma_{0} \mathbb{F}\right)\right)+\left|\mu^{+}\right|$, e

(ii) $\pi \mathbb{F}:=\pi(\mathbb{F}, V):=\left|c^{+}\right|+\left|\mu^{+}\right|+\gamma(V \backslash \check{P})-\gamma\left(\left(V \cap \Gamma_{0} \mathbb{F}\right) \backslash \check{P}\right)-\operatorname{rank}\left(M \mathbb{F} \cup \Gamma_{0} \mathbb{F}\right)$.

A prova é uma indução em sz $\mathbb{F}:=\sum[|S| \mu S \mid S \in \mu]$. Suponha que sz $\mathbb{F}=0$. Se $\mu^{+}=\emptyset$, então não há nada a provar. Se $\mu^{+} \neq \emptyset$, então $\mu^{+}=\{\emptyset\}$. Seja $y_{\emptyset}:=\overrightarrow{0}[\emptyset \mapsto \mu \emptyset]$ e $y:=\vec{\emptyset}\left[\emptyset \mapsto y_{\emptyset}\right]$. É claro que $y$ é um empacotamento que satisfaz $\left|y^{+}\right| \leqslant \min \{\varphi \mathbb{F}, \pi \mathbb{F}\}$.

Suponha, agora, que sz $\mathbb{F}>0$. Seja $S \in \mu^{+}$. Ora, $\mathbb{F}$ viável implica, em virtude do Teorema 4.7, que $\mathbb{F}$ possui uma cobertura justa $B$ de $S$ tal que $\mathbb{F}^{\alpha(B ; S) B}$ é viável. Para o resto da prova, vamos escrever

$\triangleright \quad k:=\lfloor\alpha(B ; S)\rfloor$,

ou seja, $k$ é o maior inteiro menor ou igual a $\alpha(B ; S)$. Para simplificar a notação, definimos $\mathbb{F}^{\prime}:=\mathbb{F}^{k B}$ e, de forma análoga, $c^{\prime}:=c^{k B}, p^{\prime}:=p^{k B}$ e $\mu^{\prime}:=\mu^{k B}$. Esta convenção é estendida de tal forma que sempre que rotularmos $\mathbb{F}$ com um superescrito, então os correspondentes elementos que compõe $\mathbb{F}$ também serão denotados usando-se este mesmo superescrito. É claro que $M \mathbb{F}^{\prime} \supseteq M \mathbb{F}$. Como $B$ é uma cobertura justa de $S$ em $\mathbb{F}$, então $\Gamma_{0} \mathbb{F}^{\prime} \supseteq \Gamma_{0} \mathbb{F}$ e, consequentemente, $\operatorname{rank}\left(M \mathbb{F}^{\prime} \cup \Gamma_{0} \mathbb{F}^{\prime}\right) \geqslant \operatorname{rank}\left(M \mathbb{F} \cup \Gamma_{0} \mathbb{F}\right)$. O restante da prova é dividida em dois casos, os quais passamos a descrever.

Caso $10<k=\alpha(B ; S)$.

Neste caso, sz $\mathbb{F}^{\prime}<s z \mathbb{F}$. Como $\mathbb{F}^{\prime}$ é viável, então, por hipótese de indução, $\mathbb{F}^{\prime}$ possui um empacotamento $y^{\prime}$ tal que $\left|\operatorname{supp} y^{\prime}\right| \leqslant \varphi \mathbb{F}^{\prime}$.

- Como $B$ é $S$-arbóreo, então o Lema 5.3 implica que $p^{\prime}$ é legal para $V$ em $\mathbb{F}^{\prime}$. Segue daí, como $\mathbb{F}^{\prime}$ é viável, por hipótese de indução, que $\mathbb{F}^{\prime}$ possui um empacotamento $y^{\prime}$ tal que $\left|\operatorname{supp} y^{\prime}\right| \leqslant \pi \mathbb{F}^{\prime}$

Afirmamos que

$$
\rightsquigarrow \quad \varphi \mathbb{F}^{\prime}<\varphi \mathbb{F} \quad \text { e } \quad>\pi \mathbb{F}^{\prime}<\pi \mathbb{F} \triangleleft \text {. }
$$


- Suponha, primeiro, que $k=\mu S$ ou existe $a \in B$ tal que $k=c(a)$. Então, no primeiro caso, $\left|\operatorname{supp} \mu^{\prime}\right|=\left|\mu^{+}\right|-1$, e, no segundo caso, $\left|\operatorname{supp} c^{\prime}\right|<\left|c^{+}\right|$. No entanto,

$$
\operatorname{rank}\left(M \mathbb{F}^{\prime} \cup \Gamma_{0} \mathbb{F}^{\prime}\right) \geqslant \operatorname{rank}\left(M \mathbb{F} \cup \Gamma_{0} \mathbb{F}\right)
$$

o que implica, em ambos os casos, que $\varphi \mathbb{F}^{\prime}<\varphi \mathbb{F}$ e $>\pi \mathbb{F}^{\prime}<\pi \mathbb{F}$

Suponha, agora, que $k<\mu S$ e $k<c(a)$ para cada $a \in B$. Então $k=\operatorname{cap}(w ; B, S)$ para algum $w$ especial para $B$ e $S$. Assim ${ }^{1}$,

$$
\chi^{B}(\rho w) \geqslant 2 \quad \text { ou } \quad w \notin S \text { e } \chi^{B}(\rho w)=1 .
$$

Como $\mathbb{F}^{\prime}$ é viável e $\mu^{\prime} S>0$, então o Teorema 2.8 implica que $\mathbb{F}^{\prime}$ possui uma cobertura justa $J$ de $S$. Lembre-se que $\Gamma_{0} \mathbb{F}^{\prime} \supseteq \Gamma_{0} \mathbb{F}$. Assim,

$$
\begin{aligned}
& \text { para todo } u \in \Gamma_{0} \mathbb{F}: \chi^{B}(\rho u)=\chi^{S}(u)=\chi^{J}(\rho u), \mathrm{e} \\
& \text { para todo } u \in M \mathbb{F}: \chi^{B}(\rho u)=1=\chi^{J}(\rho u) .
\end{aligned}
$$

No entanto, $w \in \Gamma_{0} \mathbb{F}^{\prime}$ e, portanto,

$$
\chi^{J}(\rho w) \leqslant 1 \quad \text { e } \quad \text { se } w \notin S \text {, então } \chi^{J}(\rho w)=0 \text {. }
$$

Finalmente, as propriedades (1०), (2०) e (3०), aliadas ao Lema 5.1 implicam que

$$
\chi^{\rho w} \notin \operatorname{span}\left(M \mathbb{F} \cup \Gamma_{0} \mathbb{F}\right)
$$

o qual, combinado com $w \in \Gamma_{0} \mathbb{F}^{\prime} \backslash \Gamma_{0} \mathbb{F}$, acarreta em $\operatorname{rank}\left(M \mathbb{F}^{\prime} \cup \Gamma_{0} \mathbb{F}^{\prime}\right)>\operatorname{rank}\left(M \mathbb{F} \cup \Gamma_{0} \mathbb{F}\right)$. Logo, $\varphi \mathbb{F}^{\prime}<\varphi \mathbb{F}$ e $\rightarrow \pi \mathbb{F}^{\prime}<\pi \mathbb{F} \varangle$, como queríamos.

Vamos agora definir um empacotamento $y$ de $\mathbb{F}$. Seja

$$
y_{S}:=y_{S}^{\prime}\left[B \mapsto y_{S}^{\prime}(B)+k\right] \quad \text { e } \quad y:=y^{\prime}\left[S \mapsto y_{S}\right]
$$

É fácil verificar que $y$ é um empacotamento de $\mathbb{F}$ tal que $|y| \leqslant\left|\operatorname{supp} y^{\prime}\right|+1$ e, portanto, $\left|y^{+}\right| \leqslant \varphi \mathbb{F}$ e $\left|y^{+}\right| \leqslant \pi \mathbb{F} \triangleleft$. Isto completa a análise do Caso 1.

Caso $2 k=0 \quad$ ou $\quad k<\alpha(B ; S)$.

Neste caso, $\mu^{\prime} S>0$. Além disso, $c^{\prime}(a)>0$ para cada $a \in B$ o que implica que $\mathbb{F}^{(k+1) B}$ é um sgnfm. No entanto, a definição de $\alpha$ implica que $\mathbb{F}^{(k+1) B}=\left(\mathbb{F}^{\prime}\right)^{B}$ não é viável. Selecione uma parte maximal $A$ de $B$ tal que

$$
A \text { é } S \text {-arbórea e } \quad\left(\mathbb{F}^{\prime}\right)^{A} \text { é viável. }
$$

\footnotetext{
${ }^{1}$ Lembre-se que $\chi^{B}(\rho w)=d_{B}(w)$. Entretanto, no argumento que segue, esta notação, embora obtusa, é mais conveniente uma vez que queremos concluir que um certo conjunto de vetores é linearmente independente.
} 
É claro que uma tal parte existe uma vez que $\emptyset$ é um candidato evidente. Como $\left(\mathbb{F}^{\prime}\right)^{B}$ não é viável, então $B \backslash A \neq \emptyset$. Se free ${ }_{S} A=\emptyset$, então seja $e$ um arco arbitrário em $B \backslash A$. Se free $_{S} A \neq \emptyset$, então seja $u$ um núcleo maximal em free ${ }_{S} A$ e tome um arco $e$ em $B \backslash A$ que cobre $u$; um tal arco existe pois $B$ cobre $u$. O Lema 2.4 implica que $J:=A \cup\{e\}$ é $S$-arbóreo. Entretanto, a maximalidade de $A$ implica que $\left(\mathbb{F}^{\prime}\right)^{J}$ não é viável. No entanto, o Teorema 2.9 implica que $\mathbb{F}^{\prime}$ possui uma cobertura justa $A^{\prime}$ de $S$ tal que

$$
A^{\prime} \supseteq A \quad \text { e } \quad\left(\mathbb{F}^{\prime}\right)^{A^{\prime}}=: \mathbb{F}^{\prime \prime} \text { é viável. }
$$

Observe que $A^{\prime} \neq B$. Além disso, sz $\mathbb{F}^{\prime \prime}<\mathrm{sz} \mathbb{F}$. Afirmamos, agora, que

$$
\rightsquigarrow \quad \operatorname{rank}\left(M \mathbb{F}^{\prime \prime} \cup \Gamma_{0} \mathbb{F}^{\prime \prime}\right)>\operatorname{rank}\left(M \mathbb{F} \cup \Gamma_{0} \mathbb{F}\right)
$$

- A viabilidade de $\mathbb{F}^{A}$ e a inviabilidade de $\mathbb{F}^{J}$ implicam na existência de $w$ em $P$ tal que

$$
e \in \rho w, \quad\left(c^{\prime}\right)^{A}(\rho w)=\left(p^{\prime}\right)^{A}(w), \quad \text { e } \quad\left(c^{\prime}\right)^{J}(\rho w)=\left(p^{\prime}\right)^{J}(w)-1 .
$$

Como $\Gamma_{0} \mathbb{F}^{\prime} \supseteq \Gamma_{0} \mathbb{F}, B$ é uma cobertura justa de $S$ em $\mathbb{F}, A^{\prime}$ é uma cobertura justa de $S$ em $\mathbb{F}^{\prime}$, e $k<\mu S$, então

$(1 \diamond) \quad$ para todo $u \in \Gamma_{0} \mathbb{F}: \chi^{B}(\rho u)=\chi^{S}(u)=\chi^{A^{\prime}}(\rho u), \mathrm{e}$ para todo $u \in M \mathbb{F}: \chi^{B}(\rho u)=1=\chi^{J}(\rho u)$.

Além disso, a escolha de $w$ implica que

$$
\chi^{A^{\prime}}(\rho w) \leqslant \chi^{B}(\rho w)-1
$$

As propriedades $(1 \diamond)$ e $(2 \diamond)$, aliadas ao Lema 5.1, implicam que $\chi^{\rho w} \notin \operatorname{span}\left(M \mathbb{F} \cup \Gamma_{0} \mathbb{F}\right)$. Finalmente, $w \in \Gamma_{0} \mathbb{F}^{\prime \prime} \backslash \Gamma_{0} \mathbb{F}$ implica que $\operatorname{rank}\left(M \mathbb{F}^{\prime \prime} \cup \Gamma_{0} \mathbb{F}^{\prime \prime}\right)>\operatorname{rank}\left(M \mathbb{F} \cup \Gamma_{0} \mathbb{F}\right)$, como queríamos.

Por hipótese de indução, $\mathbb{F}^{\prime \prime}$ possui um empacotamento $y^{\prime \prime}$ tal que $\left|\operatorname{supp} y^{\prime \prime}\right| \leqslant \varphi \mathbb{F}^{\prime \prime}$. Ponha, agora,

$$
y_{S}:=y_{S}^{\prime \prime}\left[B \mapsto y_{S}^{\prime \prime}(B)+k, A^{\prime} \mapsto y_{S}^{\prime \prime}\left(A^{\prime}\right)+1\right] \quad \text { e } \quad y:=y^{\prime \prime}\left[S \mapsto y_{S}\right] .
$$

É claro que $y$ é um empacotamento de $\mathbb{F}$. Ora, $\operatorname{rank}\left(M \mathbb{F}^{\prime \prime} \cup \Gamma_{0} \mathbb{F}^{\prime \prime}\right)>\operatorname{rank}\left(M \mathbb{F} \cup \Gamma_{0} \mathbb{F}\right)$ implica que

$$
\begin{aligned}
& \left|y^{+}\right| \leqslant\left|\operatorname{supp} y^{\prime \prime}\right|+2 \leqslant \varphi \mathbb{F}^{\prime \prime}+2 \leqslant 2\left(\left|c^{+}\right|-\operatorname{rank}\left(M \mathbb{F}^{\prime \prime} \cup \Gamma_{0} \mathbb{F}^{\prime \prime}\right)+1\right)+\left|\mu^{+}\right| \\
& \leqslant 2\left(\left|c^{+}\right|-\operatorname{rank}\left(M \mathbb{F} \cup \Gamma_{0} \mathbb{F}\right)\right)+\left|\mu^{+}\right|=\varphi \mathbb{F} .
\end{aligned}
$$

Isto completa a prova do teorema quando $\mathbb{F}$ é um sgnfm.

- Suponha, no que segue, que $\mathbb{F}$ é um sgn-c-fm. 
Note que já estabelecemos que $\operatorname{rank}\left(M \mathbb{F}^{\prime \prime} \cup \Gamma_{0} \mathbb{F}^{\prime \prime}\right)>\operatorname{rank}\left(M \mathbb{F} \cup \Gamma_{0} \mathbb{F}\right)$. Assim, se provarmos que $\gamma\left(\left(V \cap \Gamma_{0} \mathbb{F}^{\prime \prime}\right) \backslash \check{P}\right)>\gamma\left(\left(V \cap \Gamma_{0} \mathbb{F}\right) \backslash \check{P}\right)$, então $\pi \mathbb{F}^{\prime \prime} \leqslant \pi \mathbb{F}-2$. Por hipótese de indução, temos um empacotamento $y^{\prime \prime}$ de $\mathbb{F}^{\prime \prime}$ tal que $\left|\operatorname{supp} y^{\prime \prime}\right| \leqslant \pi \mathbb{F}^{\prime \prime}$. Segue daí que se utilizarmos a mesma estratégia de empacotamento que produziu $y$, então $\left|y^{+}\right| \leqslant \pi \mathbb{F}$. Vamos, pois, aos detalhes da prova.

Para abreviar, vamos escrever $\tilde{\mathbb{F}}:=\left(\mathbb{F}^{\prime}\right)^{J}$. O conjunto $X:=\{u \in P \mid \tilde{c}(u)=\tilde{p}(u)-1\}$ é não-vazio, pois $\left(\mathbb{F}^{\prime}\right)^{A}$ é viável e $\tilde{\mathbb{F}}=\left(\mathbb{F}^{\prime}\right)^{A \cup\{e\}}$ é inviável. Note que $\tilde{p}(u)>0$ para todo $u \in X$ donde $X \subseteq V$.

Observe que podemos supor que para cada arco $e$ de $B$ existe $s \in S$ tal que $e \in \rho s$ uma vez que o Teorema 4.7 naturalmente produz uma cobertura dotada desta propriedade. Esta propriedade somente será utilizada na prova do fato que segue.

Fato 5.5.1 Se $u \in X$ então $u \notin \check{P}$

- Seja $u \in X$. É claro que $e \in \rho u$. Como $e$ está em $B$, então existe $s \in S$ tal que $e \in \rho s$. Segue daí, em virtude de ( $\rho .3)$, que $u \widetilde{\bowtie} s$. Se $u \bowtie s$ ou $s \prec u$, então $u \wedge s \prec u$ e, portanto, $u \notin \check{P}$. Suponha que $u \preccurlyeq s$. Devido à pim, temos que $u \in S$. Mais ainda, $u \notin \check{S}$. De fato, se $u \in \check{S}$, então $d_{B}(u)=1$ e, consequentemente, $\tilde{c}(\rho u) \geqslant \tilde{p}(u)$, o que contraria $u \in X$. No entanto, $u \notin \check{S}$ implica $u \notin \check{P}$, como queríamos.

Fato 5.5.2 $\gamma\left(\left(V \cap \Gamma_{0} \mathbb{F}^{\prime \prime}\right) \backslash \check{P}\right)>\gamma\left(\left(V \cap \Gamma_{0} \mathbb{F}\right) \backslash \check{P}\right)$.

- Seja, agora, $L$ uma parte laminar de $\left(V \cap \Gamma_{0} \mathbb{F}\right) \backslash \check{P}$. Afirmamos que

$(1 *)$ para todo $u \in P: \tilde{c}(u) \geqslant \tilde{p}(u)-1$, e

$(2 *)$ para todo $\ell \in L: \tilde{c}(\ell)=\tilde{p}(\ell)$.

- Para a prova de $(1 *)$, seja $u \in P$. Então $\left(c^{\prime}\right)^{A}(u) \geqslant\left(p^{\prime}\right)^{A}(u)$ pois $\left(\mathbb{F}^{\prime}\right)^{A}$ é viável. Assim, $\tilde{c}(u)=\left(c^{\prime}\right)^{A}(u)-\chi^{\rho u}(e) \geqslant\left(p^{\prime}\right)^{A}(u)-1 \geqslant \tilde{p}(u)-1$.

Para a prova de $(2 *)$, seja $\ell \in L$. Como $J \subseteq B$ e $B$ é uma cobertura justa de $S$ em $\mathbb{F}$, então $d_{J}(\ell)=\chi^{\operatorname{cov}_{S} J}(\ell)$. Segue daí que

$$
\tilde{c}(\ell)=c^{\prime}(\ell)-d_{J}(\ell)=p^{\prime}(\ell)-d_{J}(\ell)=p^{\prime}(\ell)-\chi^{\operatorname{cov}_{S} J}(\ell)=\tilde{p}(\ell),
$$

o que estabelece $(2 *)$.

É claro que $(2 *)$ implica em $L \cap X=\emptyset$.

Agora, o Lema 2.1, quando aplicado ao iposet $P$, e aos conjuntos $X$ e $L$, implica que existe $t \in X$ tal que

para todo $\ell \in L_{t}$ e todo $z \in P: z \prec \ell$ implica que $\tilde{c}(z) \geqslant \tilde{p}(z)$. 
O Lema 3.1 por sua vez, implica que existe uma $t$-spi $\mathbf{f}$ tal que $L \cup\{t \vee \mathbf{f}\}$ é laminar. Afirmamos que

$$
\rightsquigarrow \quad \tilde{c}(t \vee \mathbf{f})=\tilde{p}(t \vee \mathbf{f})-1 .
$$

-• Primeiro, observe que como $B$ é $S$-arbóreo e $p$ é legal para $V$ em $\mathbb{F}$, então, pelo Lema 5.3, $p^{\prime}$ é legal para $V$ em $\mathbb{F}^{\prime}$ o que, por sua vez, aliado à $J$-arbóreo e ao Lema 5.3, implica que $\tilde{p}$ é legal para $V$ em $\tilde{\mathbb{F}}$.

A prova é por indução no comprimento de $\mathbf{f}$. O resultado é óbvio se $\mathbf{f}=\emptyset$ uma vez que $t \in X$. Suponha, então, que $\mathbf{f}=\mathbf{f}^{\prime} \cdot \ell$, onde $\mathbf{f}^{\prime} \subseteq L_{t}$ é uma $t$-spi e $\ell \in L_{t}$. Por hipótese de indução, $\tilde{c}\left(t \vee \mathbf{f}^{\prime}\right)=\tilde{p}\left(t \vee \mathbf{f}^{\prime}\right)-1$, donde $t \vee \mathbf{f}^{\prime} \in X$ e, por conseguinte, $t \vee \mathbf{f}^{\prime} \in V$. Note, também, que $\ell \in V$. Então

$$
\begin{aligned}
& \tilde{p}\left(t \vee \mathbf{f}^{\prime}\right)-1+\tilde{p}(\ell) \\
& =\tilde{c}\left(t \vee \mathbf{f}^{\prime}\right)+\tilde{c}(\ell) \\
& \geqslant \tilde{c}\left(\left(t \vee \mathbf{f}^{\prime}\right) \vee \ell\right)+\tilde{c}\left(\left(t \vee \mathbf{f}^{\prime}\right) \wedge \ell\right) \\
& \geqslant \tilde{p}\left(\left(t \vee \mathbf{f}^{\prime}\right) \vee \ell\right)-1+\tilde{p}\left(\left(t \vee \mathbf{f}^{\prime}\right) \wedge \ell\right) \\
& \geqslant \tilde{p}\left(t \vee \mathbf{f}^{\prime}\right)+\tilde{p}(\ell),
\end{aligned}
$$

onde a primeira igualdade vem da hipótese de indução e de $(2 *)$; a primeira desigualdade, da submodularidade de $\tilde{c}$; a segunda desigualdade, de $(1 *)$ e de $(3 *)$; e, a última desigualdade, vem de $\tilde{p}$ legal para $V$ em $\tilde{\mathbb{F}}$ e $t \vee \mathbf{f}^{\prime}, \ell \in V$. Assim, vale a igualdade em todas as passagens e, em particular, $\tilde{c}(t \vee \mathbf{f})=\tilde{p}(t \vee \mathbf{f})-1$, como queríamos.

Este último fato implica que $t \vee \mathbf{f} \in X$ e, consequentemente, $t \vee \mathbf{f} \in V \backslash(L \cup \check{P})$. Finalmente, $t \vee \mathbf{f} \in \Gamma \mathbb{F}^{\prime \prime}$ uma vez que $e \in \rho(t \vee \mathbf{f})$ e $\tilde{c}(t \vee \mathbf{f})=\tilde{p}(t \vee \mathbf{f})-1$ e, por conseguinte, concluímos que $\gamma\left(\left(V \cap \Gamma_{0} \mathbb{F}^{\prime \prime}\right) \backslash \check{P}\right)>\gamma\left(\left(V \cap \Gamma_{0} \mathbb{F}\right) \backslash \check{P}\right)$.

Ora, como $A^{\prime}$ é $S$-arbóreo e $p^{\prime}$ é legal para $V$ em $\mathbb{F}^{\prime}$, então o Lema 5.3 implica que $p^{\prime \prime}$ é legal para $V$ em $\mathbb{F}^{\prime \prime}$. Como sz $\mathbb{F}^{\prime \prime}<\mathrm{sz} \mathbb{F}$ e $\mathbb{F}^{\prime \prime}$ é viável, então, por hipótese de indução, $\mathbb{F}^{\prime \prime}$ possui um empacotamento $y^{\prime \prime}$ tal que $\left|\operatorname{supp} y^{\prime \prime}\right| \leqslant \pi \mathbb{F}^{\prime \prime}$. Definimos

$$
y_{S}:=y_{S}^{\prime \prime}\left[B \mapsto y_{S}^{\prime \prime}(B)+k, A^{\prime} \mapsto y_{S}^{\prime \prime}\left(A^{\prime}\right)+1\right] \quad \text { e } \quad y:=y^{\prime \prime}\left[S \mapsto y_{S}\right] .
$$

Agora, $\pi \mathbb{F}^{\prime} \leqslant \pi \mathbb{F}-2$ implica que $\left|y^{+}\right| \leqslant \pi \mathbb{F}$. Isto completa a prova de (ii)'.

Para estabelecer (ii), observe que pelo Lema 5.2, $p$ é bom para $U \mathbb{F}$ e, consequentemente, por (ii)', $\mathbb{F}$ possui um empacotamento $y$ tal que

$$
\left|y^{+}\right| \leqslant\left|c^{+}\right|+\left|\mu^{+}\right|+\gamma(U \mathbb{F} \backslash \check{P})-\gamma\left(\left(U \mathbb{F} \cap \Gamma_{0} \mathbb{F}\right) \backslash \check{P}\right)-\operatorname{rank}\left(M \mathbb{F} \cup \Gamma_{0} \mathbb{F}\right) .
$$

Isto completa a prova do teorema. 
A prova sugere um algoritmo que recebe um sgnfm $\mathbb{F}$ viável e inteiro e devolve um empacotamento de $\mathbb{F}$, dotado das propriedades do Teorema 5.5. Para estabelecer que este algoritmo tem complexidade de tempo polinomial, vamos admitir que está disponível um oráculo que dado $S \in \mu^{+}$e um subconjunto $B$ de $E$ calcula $\alpha(B ; S)$ em tempo polinomial. No que segue, exibimos o algoritmo implícito na prova do Teorema 5.5.

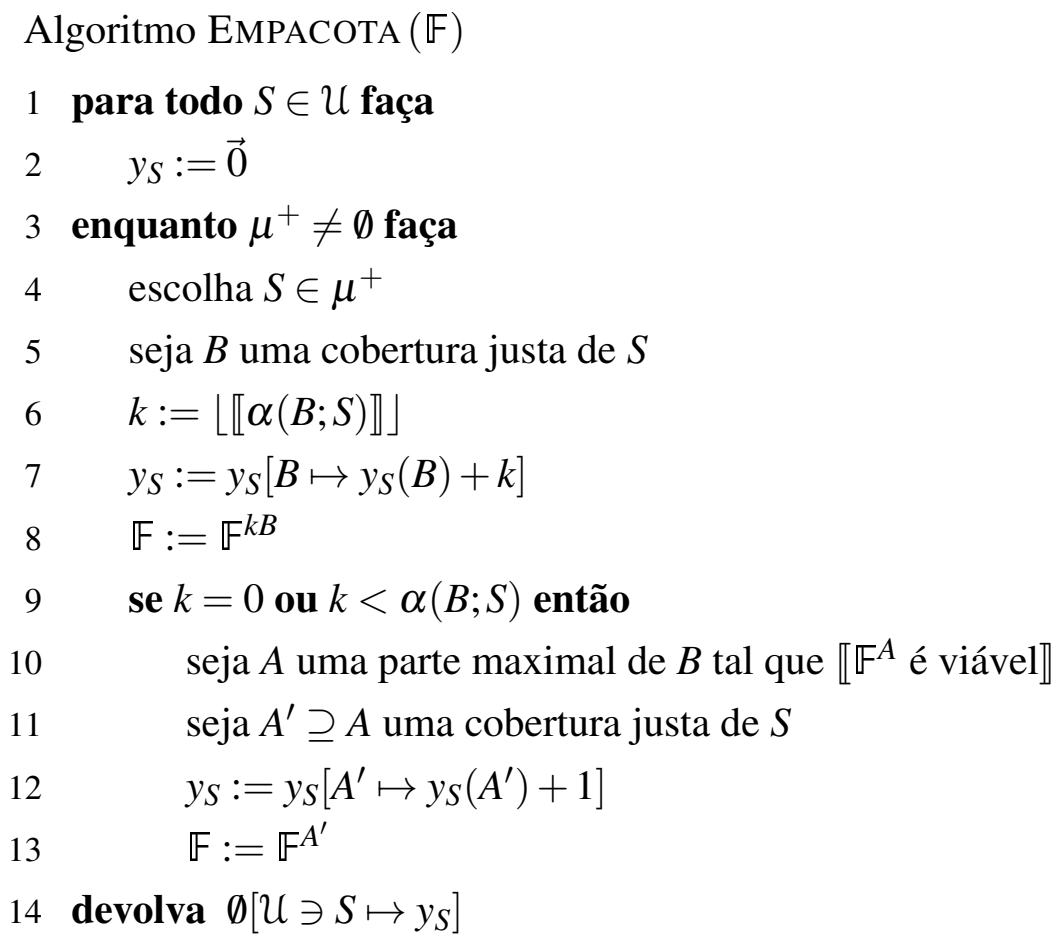

\section{Algumas consequências}

É claro que os Teoremas 5.4 e 5.5 se aplicam a cada um dos arcabouços enumerados no Capítulo 1. Vamos, nesta seção, exibir algumas consequências destes teoremas envolvendo sobretudo empacotamentos de ramificações e arborescências.

Corolário 5.6 Seja $\mathbb{K}$ um sgn-c inteiro. Então existe uma função y: $\mathcal{B}_{P} \rightarrow \mathbb{N}$ tal que

(i) $\sum\left[y(B) \mid B \in \mathcal{B}_{P}\right]=\lambda \mathbb{K}$,

(ii) $\sum\left[y(B) \chi^{B} \mid B \in \mathcal{B}_{P}\right] \leqslant c$,

(iii) $\left|y^{+}\right| \leqslant 2\left(\left|c^{+}\right|-|\check{P}|\right)+1, e$

(iv) se $\mathbb{K}$ é um sgn-c então $\left|y^{+}\right| \leqslant\left|c^{+}\right|-|\check{P}|+\gamma(P)+1$.

Prova. O resultado é evidente se $\mathbb{K}$ é trivial. Suponha, então, que $\mathbb{K}$ não é trivial. Seja $\mathbb{F}:=(\mathbb{K}, \mu)$, onde $\mu(P):=\lambda \mathbb{K}$ e $\mu(S):=0$ para cada $S \in \mathcal{U} \backslash\{P\}$. Agora, (i), (ii) e (iii) seguem do Teorema 5.5i. Para a prova de (iv), suponha que $\mathbb{K}$ é um sgn-c. Observe que o conjunto $W:=\left\{\chi^{\rho u} \mid u \in \check{P}\right\}$ é linearmente independente. De fato, suponha que $u \neq w \in \check{P}$. Ora, a propriedade $(\rho .3)$ implica que $\rho u \cap \rho w=\emptyset$. Como $\mathbb{K}$ é não-trivial então $\rho u \neq \emptyset \neq \rho w$, o que estabelece a independência linear de $W$. Finalmente, $M \mathbb{F}=\check{P}$ e $\operatorname{rank}\left(\check{P} \cup \Gamma_{0} \mathbb{F}\right) \geqslant|\check{P}|$ e o Teorema 5.5ii acarretam (iv). 
De forma análoga, usando-se o Teorema 5.4, obtemos:

Corolário 5.7 Seja $\mathbb{K}$ um sgn. Então existe uma função y: $\mathcal{B}_{P} \rightarrow \mathbb{R}_{+}$tal que

(i) $\sum\left[y(B) \mid B \in \mathcal{B}_{P}\right]=\lambda \mathbb{K}$,

(ii) $\sum\left[y(B) \chi^{B} \mid B \in \mathcal{B}_{P}\right] \leqslant c$,

(iii) $\left|y^{+}\right| \leqslant\left|c^{+}\right|-|\check{P}|+1$.

Como já mencionado, os itens (i) e (ii) do Corolário abaixo foram estabelecidos por Edmonds [Edm73]. O item (iii), que é uma pequena melhora no limitante de Gabow e Manu [GM98], segue do Corolário 5.6 observando-se que a família dos $\bar{r}$-conjuntos minimais tem cardinalidade $|V|-1$, e que cada família laminar de $\bar{r}$-conjuntos não-minimais tem cardinalidade não superior a $|V|-2$.

Corolário 5.8 Seja $D=(V, A)$ um digrafo, $c: A \rightarrow \mathbb{N}$ uma função capacidade, e $r \in V$. Seja, também, $\mathcal{B}$ o conjunto das r-arborescências geradoras de D. Então existe $y: \mathcal{B} \rightarrow \mathbb{N}$ tal que

(i) $\sum[y(B) \mid B \in \mathcal{B}]=\lambda$,

(ii) $\sum\left[y(B) \chi^{B} \mid B \in \mathcal{B}\right] \leqslant c, e$

(iii) $\left|y^{+}\right| \leqslant|A|$,

onde $\lambda:=\min \{c(\vec{\delta}(X)) \mid X$ é um $\bar{r}$-conjunto $\}$.

Vale destacar que para o empacotamento fracionário de arborescências podemos derivar, usandose o Teorema 5.4, o limitante superior $|A|-|V|+2$, que também é uma pequena melhora no limitante provado por Gabow e Manu [GM98], para o suporte do empacotamento.

\section{Empacotamento de ramificações}

Seja $D=(V, A)$ um digrafo, $c: A \rightarrow \mathbb{N}$ uma função capacidade, e $\mu: 2^{V} \rightarrow \mathbb{N}$ uma função demanda. De forma análoga ao que fizemos quando lidamos com sistemas generalizados de núcleos com uma família mista, defina para cada $X \subseteq V$ :

$\triangleright \quad p(X):=\sum\left[\mu R \mid R \in 2^{V}, R \cap X=\emptyset\right]$.

Dizemos que $(D, c, \mu)$ é viável se $c(\vec{\delta}(X)) \geqslant p(X)$ para cada $X \subseteq V$. Seja $R \in 2^{V}$, e denote por $\mathcal{B}_{R}$ o conjunto das $R$-ramificações. Uma função $y_{R}: \mathcal{B}_{R} \rightarrow \mathbb{N}$ é um empacotamento de $R$ se $\triangleright \quad \sum\left[y_{R}(B) \mid B \in \mathcal{B}_{R}\right]=\mu R$.

Uma função $y$ que atribui um empacotamento $y_{R}$ de $R$ a cada $R \in 2^{V}$ é um empacotamento de $(D, c, \mu)$ se

$\triangleright \quad \sum\left[y_{R}(B) \chi^{B} \mid R \in 2^{V}, B \in \mathcal{B}_{R}\right] \leqslant c$.

Vamos, por brevidade, denominar a tripla $(D, c, \mu)$ de rede. É fácil estabelecer que

$$
\mathbb{K}=\left(2^{V}, \subseteq, \cup, \cap, A, \vec{\delta}, c\right)
$$


é um sgn e $2^{\bar{R}}$ é $\bowtie$-fechado para cada $R \in 2^{V}$. Ademais, a família $\left\{2^{\bar{R}} \mid R \in 2^{V}, \mu R>0\right\}$ satisfaz a propriedade da intersecção mista. Portanto, $(\mathbb{K}, \mu)$ é um sgnfm. Não é difícil ver também que $(\mathbb{K}, \mu)$ é um sgn-c-fm. Assim, o seguinte corolário segue imediatamente do Teorema 5.5, o que é uma melhora no limitante provado por Schrijver [Sch03].

Corolário 5.9 Seja $\mathbb{D}=(D, c, \mu)$ uma rede viável.

Então $\mathbb{D}$ possui um empacotamento y tal que $\left|y^{+}\right| \leqslant \min \left\{2|A|+\left|\mu^{+}\right|,|A|+\left|\mu^{+}\right|+|V|-2\right\}$.

\subsection{Mais dois teoremas sobre empacotamentos}

Nesta seção, vamos provar mais dois teoremas envolvendo empacotamentos em sgnfms. Basicamente, estes teoremas fornecem um mesmo limitante que os anteriores. Entretanto, são baseados em um algoritmo distinto, que é uma generalização de um algoritmo de Schrijver [Sch03] para o empacotamento de ramificações geradoras em digrafos. A prova que apresentamos também fornece um melhor limitante - tanto para o número de iterações quanto para o tamanho do empacotamento - que o originalmente estabelecido por Schrijver [Sch03].

$\mathrm{O}$ algoritmo de Schrijver, que produz um empacotamento de ramificações geradoras em digrafos, consiste em selecionar um arco seguro e recursivamente encontrar um empacotamento no digrafo obtido ao removermos um certo número de cópias deste arco. Como já mencionado, o algoritmo de Schrijver realiza, para um digrafo $D=(V, A)$ e uma família $\mathcal{R}$ de conjuntos de raízes, $|V|^{3}+|A|+|\mathcal{R}|$ iterações e, evidentemente, produz um empacotamento de tamanho não superior a este número. O próximo teorema, quando particularizado para o problema de empacotamentos de ramificações geradoras em digrafos, mostra que o número de iterações e o tamanho do empacotamento estão limitados por $|A|+|\mathcal{R}|(2|V|-1)$. Vamos, posteriormente, melhorar este limitante para $|A|+2|V|+|\mathcal{R}|$.

Teorema 5.10 Seja $\mathbb{F}$ um sgnfm viável. Então existe um empacotamento y de $\mathbb{F}$ tal que

$$
\left|y^{+}\right| \leqslant\left|c^{+}\right|+\left|\mu^{+}\right|+\sum\left[\left(\left|c^{+}\right|-\operatorname{rank}\left(\Gamma_{0} \mathbb{F} \backslash S\right)\right) \mid S \in \widehat{\mu^{+}}\right] .
$$

Ademais, se $\mathbb{F}$ é inteiro, então y é inteiro.

Prova. Para simplificar, vamos escrever

$$
\begin{aligned}
& \triangleright \varphi_{0} \mathbb{F}:=\sum\left[\left(\left|c^{+}\right|-\operatorname{rank}\left(\Gamma_{0} \mathbb{F} \backslash S\right)\right) \mid S \in \widehat{\mu^{+}}\right], \mathrm{e} \\
& \triangleright \varphi \mathbb{F}:=\left|c^{+}\right|+\left|\mu^{+}\right|+\varphi_{0} \mathbb{F} .
\end{aligned}
$$

A prova é por indução em sz $\mathbb{F}:=\sum[|S| \mu(S) \mid S \in \mu]$. Suponha, primeiro, que sz $\mathbb{F}=0$. O resultado é trivial se $\mu^{+}=\emptyset$. Se $\mu^{+} \neq \emptyset$, então $\emptyset \in \mu^{+}$. Neste caso, seja $y_{\emptyset}:=\overrightarrow{0}[\emptyset \mapsto \mu \emptyset]$ e $y:=\vec{\emptyset}\left[\emptyset \mapsto y_{\emptyset}\right]$. É claro que $y$ é um empacotamento de $\mathbb{F}$ que satisfaz (i) e (ii).

Suponha, agora, que sz $\mathbb{F}>0$. Então existe $S \in \widehat{\mu^{+}}$tal que $S \neq \emptyset$. O Lema 4.6 implica que $(\Gamma \mathbb{F} \backslash S, S, \emptyset, \infty)$ é evitável. Segue daí, usando-se a Proposição 2.6, que existe um arco $a \in c^{+}$ que cobre algum núcleo de $S$, evita $\Gamma \mathbb{F} \backslash S$ e, ademais, $\{a\}$ é $S$-arbóreo. Como $a \in c^{+}$então, evidentemente, $a$ evita $\Gamma_{0} \mathbb{F} \backslash S$. Seja 
$\triangleright \quad k:=\min (\{\mu S, c(a)\} \cup\{c(u)-p(u) \mid u \in \bar{S} ; a \in \rho u\})$.

Ponha, para abreviar, $\mathbb{F}^{\prime}:=\mathbb{F}^{k a}$. Em virtude do Corolário 4.3, temos que $\mathbb{F}^{\prime}$ é um sgnfm

Fato 5.10.1 $\mathbb{F}^{\prime}$ é viável.

- De fato, note primeiro que $c^{\prime} \geqslant 0$ e $\mu^{\prime} \geqslant 0$. Ora, se $a \notin \rho u$, então não há nada a provar. Suponha, assim, que $a \in \rho u$. Se $u \in S$, então

$$
c^{\prime}(\rho u)=c(u)-k \geqslant p(u)-k=p^{\prime}(u) .
$$

Suponha, agora, que $u \notin S$. Como $a$ evita $\Gamma_{0} \mathbb{F} \backslash S$, então $c(u)>p(u)$. A definição de $k$ implica que $k \leqslant c(u)-p(u)$. Segue daí que $c^{\prime}(\rho u)=c(u)-k \geqslant p(u)=p^{\prime}(u)$, como queríamos.

Observe que $\left|\operatorname{supp} c^{\prime}\right| \leqslant\left|c^{+}\right|$. Ademais, $\mathbb{F}^{\prime}$ viável implica $\Gamma_{0} \mathbb{F}^{\prime} \supseteq \Gamma_{0} \mathbb{F}$. Seja $S^{\circ}:=$ free $_{S}\{a\}$.

Fato 5.10.2 $\varphi_{0} \mathbb{F}^{\prime} \leqslant \varphi_{0} \mathbb{F}$.

- Se $S \in \widehat{\operatorname{supp} \mu^{\prime}}$, então $\widehat{\operatorname{supp} \mu^{\prime}}=\widehat{\mu^{+}}$o que, quando combinado com $\Gamma_{0} \mathbb{F}^{\prime} \supseteq \Gamma_{0} \mathbb{F}$, acarreta em $\varphi_{0} \mathbb{F}^{\prime} \leqslant \varphi_{0} \mathbb{F}$. Suponha, assim, que $S \notin \widehat{\operatorname{supp} \mu^{\prime}}$ (ou seja, $\mu^{\prime}(S)=0$ ). Como $S^{\circ} \in \operatorname{supp} \mu^{\prime}$, então existe $T \in \widehat{\operatorname{supp} \mu^{\prime}}$ tal que $T \supseteq S^{\circ}$. Se $T \in \widehat{\mu^{+}}$, então $\widehat{\operatorname{supp} \mu^{\prime}} \subseteq \widehat{\mu^{+}}$, donde, mais uma vez, $\varphi_{0} \mathbb{F}^{\prime} \leqslant \varphi_{0} \mathbb{F}$. Suponha que $T \notin \widehat{\mu^{+}}$. Então $T \subseteq S$ o que, aliado à $\Gamma_{0} \mathbb{F}^{\prime} \supseteq \Gamma_{0} \mathbb{F}$, implica que

$$
\operatorname{rank}\left(\Gamma_{0} \mathbb{F}^{\prime} \backslash T\right) \geqslant \operatorname{rank}\left(\Gamma_{0} \mathbb{F} \backslash S\right)
$$

e, consequentemente, $\varphi_{0} \mathbb{F}^{\prime} \leqslant \varphi_{0} \mathbb{F}$.

Como a cobre algum elemento de $S$, temos que $S^{\circ} \subset S$. Ora, $S^{\circ} \subset S$ acarreta em sz $\mathbb{F}^{\prime}<$ sz $\mathbb{F}$. Mas, $\mathbb{F}^{\prime}$ é viável. Assim, por hipótese de indução, existe um empacotamento $y^{\prime}$ de $\mathbb{F}^{\prime}$ tal que $\left|\operatorname{supp} y^{\prime}\right| \leqslant \varphi \mathbb{F}^{\prime}$. Note que $\mu^{\prime}\left(S^{\circ}\right) \geqslant k$.

Fato 5.10.3 Existe um empacotamento $y$ de $\mathbb{F}$ tal que

(i) $\mu^{\prime}\left(S^{\circ}\right)=k$ implica $\left|y^{+}\right|=\left|\operatorname{supp} y^{\prime}\right|$, e

(ii) $\mu^{\prime}\left(S^{\circ}\right)>k$ implica $\left|y^{+}\right|=\left|\operatorname{supp} y^{\prime}\right|+1$.

- Sejam $B_{1}, \ldots, B_{e}$ as coberturas de $\operatorname{supp}\left(y_{S^{\circ}}^{\prime}\right)$. Então $\sum\left[y_{S^{\circ}}^{\prime}\left(B_{i}\right) \mid i \in[e]\right]=\mu^{\prime}\left(S^{\circ}\right) \geqslant k$. Escolha $i$ em $[e]$ tal que

$$
\sum\left[y_{S^{\circ}}^{\prime}\left(B_{j}\right) \mid j \in[i-1]\right]<k \quad \text { e } \quad \sum\left[y_{S^{\circ}}^{\prime}\left(B_{j}\right) \mid j \in[i]\right] \geqslant k .
$$

Ponha $k^{\prime}:=k-\sum\left[y_{S^{\circ}}^{\prime}\left(B_{j}\right) \mid j \in[i-1]\right]$. Defina, agora, $y_{S}: \mathcal{B}_{S} \rightarrow \mathbb{R}_{+}$pondo-se $\left.\triangleright \quad y_{S}:=\overrightarrow{0}\left[\forall j \in[i-1]:\{a\} \cup B_{j} \mapsto y_{S}^{\prime}\left(\{a\} \cup B_{j}\right)+y_{S^{\circ}}^{\prime}\left(B_{j}\right)\right),\{a\} \cup B_{i} \mapsto y_{S}^{\prime}\left(\{a\} \cup B_{i}\right)+k^{\prime}\right]$

Além disso, seja

$\triangleright \quad y_{S^{\circ}}:=\overrightarrow{0}\left[B_{i} \mapsto y_{S^{\circ}}^{\prime}\left(B_{i}\right)-k^{\prime}, \forall j \in\{i+1, \ldots, e\}: B_{j} \mapsto y_{S^{\circ}}^{\prime}\left(B_{j}\right)\right]$.

Finalmente, seja $y:=\vec{\emptyset}\left[S \mapsto y_{S}, S^{\circ} \mapsto y_{S^{\circ}},(\operatorname{supp} \mu) \backslash\left\{S, S^{\circ}\right\} \ni I \mapsto y_{I}^{\prime}\right]$. É fácil verificar que $y$ é um empacotamento de $\mathbb{F}$ que satisfaz (i) e (ii). 
Vamos, agora, levando-se em conta a definição de $k$, separar a prova nos seguintes casos:

Caso $1 k=\mu S$.

Neste caso, $S \notin \operatorname{supp}\left(\mu^{\prime}\right)$. Suponha que $S^{\circ} \in \mu^{+}$, donde $\mu^{\prime}\left(S^{\circ}\right)>k$. Assim, $\left|\operatorname{supp} \mu^{\prime}\right|<\left|\mu^{+}\right|$ o que, aliado à $\varphi_{0} \mathbb{F}^{\prime} \leqslant \varphi_{0} \mathbb{F}$, fornece $\varphi \mathbb{F}^{\prime}<\varphi \mathbb{F}$. Segue daí, usando-se o Fato 5.10.3ii, que existe um empacotamento $y$ de $\mathbb{F}$ tal que $\left|y^{+}\right| \leqslant \mid$supp $y^{\prime} \mid+1$ e, consequentemente, $\left|y^{+}\right| \leqslant \varphi \mathbb{F}$. Suponha, agora, que $S^{\circ} \notin \mu^{+}$, donde $\mu^{\prime}\left(S_{0}\right)=k$. Neste caso, $\left|\operatorname{supp} \mu^{\prime}\right|=|\operatorname{supp} \mu|$ e, daí, $\varphi \mathbb{F}^{\prime} \leqslant \varphi \mathbb{F}$. Finalmente, usando-se o Fato 5.10.3i, vem que existe um empacotamento $y$ de $\mathbb{F}$ tal que $\left|y^{+}\right| \leqslant\left|\operatorname{supp} y^{\prime}\right|$ e, assim, $\left|y^{+}\right| \leqslant \varphi \mathbb{F}$, o que conclui a análise do Caso 1 .

Caso $2 k<\mu S$ e $k=c(a)$.

Neste caso, $\left|\operatorname{supp} c^{\prime}\right|<\left|c^{+}\right|$. Suponha, primeiro, que $S^{\circ} \in \mu^{+}$, donde $\left|\operatorname{supp} \mu^{\prime}\right|=\left|\mu^{+}\right|$. Logo, $\varphi \mathbb{F}^{\prime}<\varphi \mathbb{F}$. Agora, o Fato 5.10 .3 implica na existência de um empacotamento $y$ de $\mathbb{F}$ tal que $\left|y^{+}\right| \leqslant\left|\operatorname{supp} y^{\prime}\right|+1$ o que, combinado com $\varphi \mathbb{F}^{\prime}<\varphi \mathbb{F}$, fornece $\left|y^{+}\right| \leqslant \varphi \mathbb{F}$.

Suponha, agora, que $S^{\circ} \notin \mu^{+}$. Então $\left|\operatorname{supp} \mu^{\prime}\right|=\left|\mu^{+}\right|+1$ e $\mu^{\prime}\left(S^{\circ}\right)=k$. Portanto, $\varphi \mathbb{F}^{\prime} \leqslant \varphi \mathbb{F}$. Assim, pelo Fato 5.10.3, existe um empacotamento $y$ de $\mathbb{F}$ tal que $\left|y^{+}\right| \leqslant\left|\operatorname{supp} y^{\prime}\right|$ o que, combinado com $\varphi \mathbb{F}^{\prime} \leqslant \varphi \mathbb{F}$, fornece $\left|y^{+}\right| \leqslant \varphi \mathbb{F}$, completando a análise do Caso 2 .

Caso $3 k<\mu S$ e $k<c(a)$.

Então $k=c(w)-p(w)$ para algum $w \in \bar{S}$. Note que como $k<c(a)$ então $p(w)>0$ e, daí, $w \in U(\mathbb{F})$. Como $a$ cobre $w$ e $a \in c^{+}$, então $w \notin \Gamma_{0} \mathbb{F} \backslash S$ e, portanto, $w \notin \Gamma_{0} \mathbb{F} \cup S$. Mas, $c^{\prime}(w)=p^{\prime}(w)$ e, por conseguinte, $w \in \Gamma_{0} \mathbb{F}^{\prime} \backslash S$. Como $a$ evita $\Gamma_{0} \mathbb{F} \backslash S$, então para cada $u \in \Gamma_{0} \mathbb{F} \backslash S:$

(i) $c(u)=p(u)$, e

(ii) $c^{\prime}(u)=c(u)=p(u)$.

Ademais, $c(w)>p(w)$ e $c^{\prime}(w)=p^{\prime}(w)=p(w)$. Pelo Lema 5.1, $\chi^{\rho w} \notin \operatorname{span}\left(\Gamma_{0} \mathbb{F} \backslash S\right)$, donde $\operatorname{rank}\left(\Gamma_{0} \mathbb{F}^{\prime} \backslash S\right)>\operatorname{rank}\left(\Gamma_{0} \mathbb{F} \backslash S\right)$ e, consequentemente, $\varphi_{0} \mathbb{F}^{\prime}<\varphi_{0} \mathbb{F}$. Se $S^{\circ} \in \mu^{+}$, então $\left|\operatorname{supp} \mu^{\prime}\right|=\left|\mu^{+}\right|$e, assim, $\varphi \mathbb{F}^{\prime}<\varphi \mathbb{F}$. O Fato 5.10 .3 implica que existe um empacotamento $y$ de $\mathbb{F}$ tal que $\left|y^{+}\right| \leqslant \varphi \mathbb{F}$. Finalmente, se $S^{\circ} \notin \mu^{+}$, então $\mu^{\prime}\left(S^{\circ}\right)=k$; mais ainda, $\left|\operatorname{supp} \mu^{\prime}\right|=\left|\mu^{+}\right|+1$ e, consequentemente, $\varphi \mathbb{F}^{\prime} \leqslant \varphi \mathbb{F}$. Logo, pelo Fato 5.10.3, existe um empacotamento $y$ de $\mathbb{F}$ tal que $\left|y^{+}\right| \leqslant \varphi \mathbb{F}$.

É claro que se $\mathbb{F}$ é inteiro, então y é inteiro. Isto completa a prova do teorema.

Vamos, agora, ao segundo teorema. Como é de se esperar, este envolve sgn-c-fm e, mais uma vez, vamos utilizar a natureza mais restrita dos sgn-c-fm, e a possibilidade de lidar com um conjunto laminar, para produzir um melhor limitante para o tamanho de um empacotamento. O próximo teorema implica num limitante de $|A|+2|V|+|\mathcal{R}|$ para o número de iterações e para o tamanho do empacotamento produzido pelo algoritmo de Schrijver. 
Proposição 5.11 Seja $\mathbb{F}$ um sgn-c-mf $e V$ uma parte de P. Se $\mathbb{F}$ é viável e inteiro, e p é legal para $V$, então $\mathbb{F}$ possui um empacotamento inteiro y tal que $\left|y^{+}\right| \leqslant\left|c^{+}\right|+\left|\mu^{+}\right|+\gamma V-\gamma\left(V \cap \Gamma_{0} \mathbb{F}\right)$.

Prova. Suponha que $\mathbb{F}$ é viável e inteiro e que $p$ é legal para $V$. Para simplificar, vamos escrever $\triangleright \quad \pi(\mathbb{F}, V):=\left|c^{+}\right|+\left|\mu^{+}\right|+\gamma V-\gamma\left(V \cap \Gamma_{0} \mathbb{F}\right)$.

A prova é por indução em sz $\mathbb{F}:=\sum[|S| \mu S \mid S \in \mu]$. Suponha, primeiro, que sz $\mathbb{F}=0$. O resultado é trivial se $\mu=\emptyset$. Se $\mu^{+} \neq \emptyset$, então $\emptyset \in \mu^{+}$. Neste caso, seja $y_{\emptyset}:=\overrightarrow{0}[\emptyset \mapsto \mu \emptyset]$ e $y:=\vec{\emptyset}\left[\emptyset \mapsto y_{\emptyset}\right]$. É claro que $y$ é um empacotamento inteiro de $\mathbb{F}$ tal que $\left|y^{+}\right| \leqslant \pi \mathbb{F}$.

Suponha, agora, que sz $\mathbb{F}>0$. Então existe $S \in \mu^{+}$tal que $S \neq \emptyset$. O Lema 4.6 implica que

$$
(\Gamma \mathbb{F} \backslash S, S, \emptyset, \infty)
$$

é evitável. Segue daí, usando-se a Proposição 2.6, que existe um arco $a \in c^{+}$que cobre algum núcleo de $S$, evita $\Gamma \mathbb{F} \backslash S$ e, ademais, $\{a\}$ é $S$-arbóreo. Como $a \in c^{+}$, então, evidentemente, $a$ evita $\Gamma_{0} \mathbb{F} \backslash S$. Seja

$\triangleright \quad k:=\min (\{\mu S, c(a)\} \cup\{c(u)-p(u) \mid u \in \bar{S} ; a \in \rho u\})$.

Para abreviar, no que segue, vamos escrever $\mathbb{F}^{\prime}:=\mathbb{F}^{k a}$ e, naturalmente, os membros de $\mathbb{F}^{\prime}$ serão denotados de forma similar. Ora, $\{a\}$ é $S$-arbóreo e, daí, o Corolário 4.3 implica que $\mathbb{F}^{\prime}$ é um sgn-c-fm. Ademais, pelo Fato 5.10.1, $\mathbb{F}^{\prime}$ é viável.

Observe que $\mid$ supp $c^{\prime}|\leqslant| c^{+} \mid$. Ademais, $\mathbb{F}^{\prime}$ viável implica $\Gamma_{0} \mathbb{F}^{\prime} \supseteq \Gamma_{0} \mathbb{F}$. Seja $S^{\circ}:=$ free $_{S}\{a\}$. Como a cobre algum elemento de $S$, temos que $S^{\circ} \subset S$ e, consequentemente, sz $\mathbb{F}^{\prime}<$ sz $\mathbb{F}$. Pelo Lema 5.3, como $\{a\}$ é $S$-arbóreo e $p$ é legal para $V$, então $p^{\prime}$ é legal para $V$ em $\mathbb{F}^{\prime}$. Assim, por hipótese de indução, existe um empacotamento $y^{\prime}$ de $\mathbb{F}^{\prime}$ tal que $\left|\operatorname{supp} y^{\prime}\right| \leqslant \pi\left(\mathbb{F}^{\prime}, V\right)$.

Note que $\mu^{\prime}\left(S^{\circ}\right) \geqslant k$. Vamos, agora, levando-se em conta a definição de $k$, separar a prova nos seguintes casos:

Caso $1 k=\mu S$.

Neste caso, $S \notin \operatorname{supp} \mu^{\prime}$. Suponha que $S^{\circ} \in \mu^{+}$, donde $\mu^{\prime}\left(S^{\circ}\right)>k$. Assim, $\left|\operatorname{supp} \mu^{\prime}\right|<\left|\mu^{+}\right|$o que, aliado à $\gamma\left(V \cap \Gamma_{0} \mathbb{F}^{\prime}\right) \geqslant \gamma\left(V \cap \Gamma_{0} \mathbb{F}\right)$, fornece $\pi\left(\mathbb{F}^{\prime}, V\right)<\pi(\mathbb{F}, V)$ O Fato 5.10.3ii implica que existe um empacotamento $y$ de $\mathbb{F}$ tal que $\left|y^{+}\right| \leqslant\left|\operatorname{supp} y^{\prime}\right|+1$ e, daí, $\left|y^{+}\right| \leqslant \pi \mathbb{F}$.

Suponha, agora, que $S^{\circ} \notin \mu^{+}$donde $\mu^{\prime}\left(S^{\circ}\right)=k$. Neste caso, $\left|\operatorname{supp} \mu^{\prime}\right|=\left|\mu^{+}\right|$e, consequentemente, $\pi\left(\mathbb{F}^{\prime}, V\right) \leqslant \pi(\mathbb{F}, V)$. Finalmente, usando-se o Fato 5.10.3i, vem que existe um empacotamento $y$ de $\mathbb{F}$ tal que $\left|y^{+}\right| \leqslant \mid$supp $y^{\prime} \mid$ e, assim, $\left|y^{+}\right| \leqslant \pi \mathbb{F}$, completando a análise do Caso 1.

Caso $2 k<\mu S$ e $k=c(a)$.

Neste caso, $\left|\operatorname{supp} c^{\prime}\right|<\left|c^{+}\right|$. Suponha, primeiro, que $S^{\circ} \in \mu^{+}$, donde $\left|\operatorname{supp} \mu^{\prime}\right|=\left|\mu^{+}\right|$e, assim, $\pi \mathbb{F}^{\prime}<\pi \mathbb{F}$. O Fato 5.10.3 implica na existência de um empacotamento $y$ de $\mathbb{F}$ tal que $\left|y^{+}\right| \leqslant\left|\operatorname{supp} y^{\prime}\right|+1$ o que, combinado com $\pi \mathbb{F}^{\prime}<\pi \mathbb{F}$, fornece $\left|y^{+}\right| \leqslant \pi \mathbb{F}$. 
Suponha, agora, que $S^{\circ} \notin \mu^{+}$. Por conseguinte, $\mid$supp $\mu^{\prime}|=| \mu^{+} \mid+1$ e $\mu^{\prime}\left(S^{\circ}\right)=k$ e, assim, $\pi\left(\mathbb{F}^{\prime}, V\right) \leqslant \pi(\mathbb{F}, V)$. O Fato 5.10 .3 implica na existência de um empacotamento $y$ de $\mathbb{F}$ tal que $\left|y^{+}\right| \leqslant\left|\operatorname{supp} y^{\prime}\right|$ o que, combinado com $\pi\left(\mathbb{F}^{\prime}, V\right) \leqslant \pi(\mathbb{F}, V)$, fornece $\left|y^{+}\right| \leqslant \pi(\mathbb{F}, V)$, completando a análise do Caso 2.

Caso $3 k<\mu S$ e $k<c(a)$.

Neste caso, $k=c(w)-p(w)$ para algum $w \in \bar{S}$ tal que $a \in \rho(w)$. Para encurtar, vamos escrever $\tilde{\mathbb{F}}:=\mathbb{F}^{(k+1) a}$. Note que $\tilde{\mathbb{F}}$ é um sgn-c-fm. Uma vez mais, pelo Lema 5.3 , como $p$ é legal para $V$, então $\tilde{p}$ é legal para $V$ em $\tilde{\mathbb{F}}$. Observe que $k<c(a)$ e $c$ inteiro implicam que $\tilde{c} \geqslant 0$. Ora, $\tilde{\mathbb{F}}$ é inviável e, desta forma, o conjunto

$$
X:=\{u \in P \mid \tilde{c}(u)=\tilde{p}(u)-1\}
$$

é não-vazio. É claro que $X \subseteq \bar{S}$. Além disso, $\tilde{p}(u)>0$ para todo $u \in X$ e, portanto, $X \subseteq V$. Seja $L$ uma parte laminar de $V \cap \Gamma_{0} \mathbb{F}$. Então

$(1 \diamond)$ para todo $u \in P: \tilde{c}(u) \geqslant \tilde{p}(u)-1, \mathrm{e}$

$(2 \diamond)$ para todo $\ell \in L: \tilde{c}(\ell) \geqslant \tilde{p}(\ell)$.

- Eis a prova de $(1 \diamond)$ e $(2 \diamond)$. Lembre-se que $\mathbb{F}$ é viável. Seja $u \in P$. Se $a \notin \rho u$, então não há nada a provar. Suponha, assim, que $a \in \rho u$. Se $u \in S$, então

$$
\tilde{c}(u)=c(u)-(k+1) \geqslant p(u)-(k+1)=\tilde{p}(u) .
$$

Se $u \in \bar{S}$, então $\tilde{c}(u)=c(u)-(k+1) \geqslant p(u)+k-(k+1)=p(u)-1=\tilde{p}(u)$, uma vez que, pela escolha de $k, c(u) \geqslant p(u)+k$. Isto prova $(1 \diamond)$. Note que já estabelecemos que $\tilde{c}(s) \geqslant \tilde{p}(s)$ para todo $s \in S$. Assim, suponha, agora, que $u \in \Gamma_{0} \mathbb{F} \backslash S$. Então $a \notin \rho u$, pois $a$ evita $\Gamma_{0} \mathbb{F} \backslash S$ e, daí, $\tilde{c}(u)=c(u) \geqslant p(u)=\tilde{p}(u)$. Segue daí que $\tilde{c}(s) \geqslant \tilde{p}(s)$ para todo $s \in \Gamma_{0} \mathbb{F}$, o que estabelece a validade de $(2 \diamond)$.

Note que $(2 \diamond)$ implica que $L \cap X=\emptyset$.

Agora, o Lema 2.1, quando aplicado ao iposet $P$, e aos conjuntos $X$ e $L$, implica que existe $t \in X$ tal que

$$
\text { para todo } \ell \in L_{t} \text { e todo } z \in P: z \prec \ell \text { implica } \tilde{c}(z) \geqslant \tilde{p}(z) \text {. }
$$

O Lema 3.1, por sua vez, implica que existe uma $t$-spi f tal que $L \cup\{t \vee \mathbf{f}\}$ é laminar.

Fato 5.11.1 $\tilde{c}(t \vee \mathbf{f})=\tilde{p}(t \vee \mathbf{f})-1$.

- A prova é por indução no comprimento de $\mathbf{f}$. O resultado é óbvio se $\mathbf{f}=\emptyset$ uma vez que $t \in X$. Suponha, então, que $\mathbf{f}=\mathbf{f}^{\prime} \cdot \ell$, onde $\mathbf{f}^{\prime} \subseteq L_{t}$ é uma $t$-spi e $\ell \in L_{t}$. Por hipótese de indução, $\tilde{c}\left(t \vee \mathbf{f}^{\prime}\right)=\tilde{p}\left(t \vee \mathbf{f}^{\prime}\right)-1$ donde $t \vee \mathbf{f}^{\prime} \in X$ e, por conseguinte, $t \vee \mathbf{f}^{\prime} \in V$. Note, também, que $\ell \in V$. Então 


$$
\begin{aligned}
& \tilde{p}\left(t \vee \mathbf{f}^{\prime}\right)-1+\tilde{p}(\ell) \\
& =\tilde{c}\left(t \vee \mathbf{f}^{\prime}\right)+\tilde{c}(\rho \ell) \\
& \geqslant \tilde{c}\left(\left(t \vee \mathbf{f}^{\prime}\right) \vee \ell\right)+\tilde{c}\left(\left(t \vee \mathbf{f}^{\prime}\right) \wedge \ell\right) \\
& \geqslant \tilde{p}(t \vee \mathbf{f})-1+\tilde{p}\left(\left(t \vee \mathbf{f}^{\prime}\right) \wedge \ell\right) \\
& \geqslant \tilde{p}\left(t \vee \mathbf{f}^{\prime}\right)+\tilde{p}(\ell) .
\end{aligned}
$$

Assim, vale a igualdade em todas as passagens e, em particular, $\tilde{c}(t \vee \mathbf{f})=\tilde{p}(t \vee \mathbf{f})-1$, como queríamos.

Este último fato implica que $t \vee \mathbf{f} \in X$ donde $t \vee \mathbf{f} \in V \backslash(S \cup L)$. Finalmente, $t \vee \mathbf{f} \in \Gamma \mathbb{F}^{\prime}$ uma vez que $a \in \rho(t \vee \mathbf{f})$ e $\tilde{c}(t \vee \mathbf{f})=\tilde{p}(t \vee \mathbf{f})-1$. Isto estabelece que $\gamma\left(V \cap \Gamma_{0} \mathbb{F}^{\prime}\right)>\gamma\left(V \cap \Gamma_{0} \mathbb{F}\right)$. Suponha, primeiro, que $S^{\circ} \in \mu^{+}$donde $\left|\operatorname{supp} \mu^{\prime}\right|=\left|\mu^{+}\right|$. Desta forma, $\pi\left(\mathbb{F}^{\prime}, V\right)<\pi(\mathbb{F}, V)$. Assim, pelo Fato 5.10.3, existe um empacotamento $y$ de $\mathbb{F}$ tal que $\left|y^{+}\right| \leqslant\left|\operatorname{supp} y^{\prime}\right|+1$ o que, combinado com $\pi \mathbb{F}^{\prime}<\pi \mathbb{F}$, fornece $\left|y^{+}\right| \leqslant \pi \mathbb{F}$. Finalmente, se $S^{\circ} \notin \mu^{+}$então $\mu^{\prime}\left(S^{\circ}\right)=$ $k$; mais ainda, $\left|\operatorname{supp} \mu^{\prime}\right|=\left|\mu^{+}\right|+1$ e, consequentemente, $\pi\left(\mathbb{F}^{\prime}, V\right) \leqslant \pi(\mathbb{F}, V)$. Logo, pelo Fato 5.10.3, existe um empacotamento $y$ de $\mathbb{F}$ tal que $\left|y^{+}\right| \leqslant \pi \mathbb{F}$. Isto completa a análise do Caso 3.

É claro que se $\mathbb{F}$ é inteiro, então $y$ é inteiro, o que. finaliza a prova do teorema.

Teorema 5.12 Se $\mathbb{F}$ é um $\mathbf{s g n - c - f m ~ v i a ́ v e l ~ e ~ i n t e i r o , ~ e n t a ̃ o ~} \mathbb{F}$ possui um empacotamento y tal que

$$
\left|y^{+}\right| \leqslant\left|c^{+}\right|+\left|\mu^{+}\right|+\gamma(U \mathbb{F})-\gamma(\Gamma \mathbb{F}) .
$$

Prova. Seja $\mathbb{F}$ um sgn-c-fm. Pelo Lema 5.2, p é bom para $U \mathbb{F}$ e, daí, pela Proposição 5.11, $\mathbb{F}$ possui um empacotamento $y$ tal que $\left|y^{+}\right| \leqslant\left|c^{+}\right|+\left|\mu^{+}\right|+\gamma(U \mathbb{F})-\gamma\left(U \mathbb{F} \cap \Gamma_{0} \mathbb{F}\right)$.

Implícito na prova dos Teoremas 5.10 e 5.12 está o algoritmo cuja descrição segue. Note que para garantir o limitante do Teorema 5.12 não é necessário selecionar, na linha $6, S$ maximal em $\mu^{+}$; basta selecionar um elemento arbitrário de $\mu^{+}$.

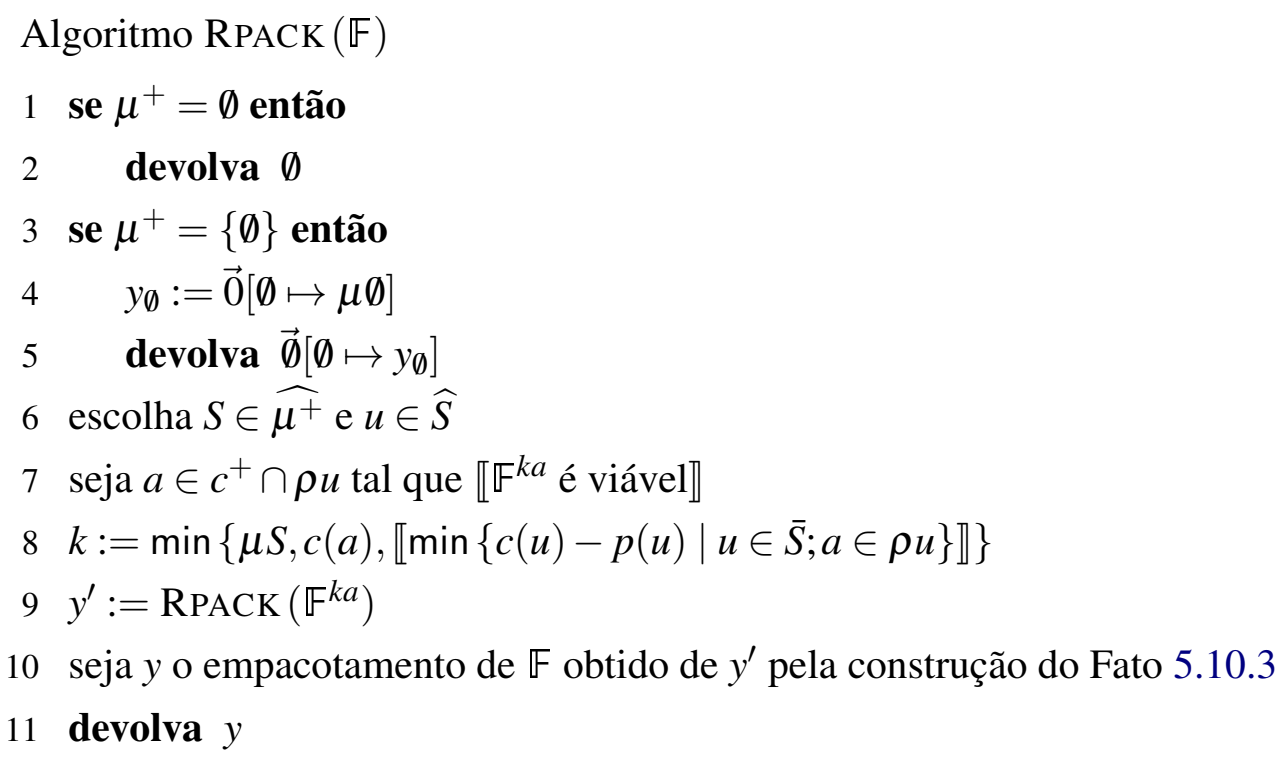




\section{Capítulo 6}

\section{Alguns poliedros}

Neste capítulo, vamos provar uma caracterização para o politopo das coberturas de um sistema generalizado de núcleos. Além disso, vamos estabelecer a integralidade de um certo poliedro, cuja técnica de prova fornecerá um algoritmo polinomial, módulo um oráculo de separação, para um certo problema de minimização, que generaliza, dentre outros, o problema da arborescência geradora de custo mínimo.

\subsection{Uma caracterização do politopo das coberturas}

Nesta seção, vamos provar uma caracterização para o politopo das coberturas de um sistema generalizado de núcleos. Para isso, seja $\mathbb{K}$ um sgn, cujo conjunto de núcleos é, como de hábito, denotado por $P$. O politopo das coberturas de $P$, boas para $\check{P}$, é o fecho convexo dos vetores de incidência das coberturas de $P$, boas para $\check{P}$, isto é,

$\triangleright \quad \mathrm{P} \mathbb{K}:=\operatorname{conv}\left\{\chi^{B} \mid B\right.$ é uma cobertura de $P$, boa para $\left.\check{P}\right\}$

Teorema 6.1 (Frank [Fra99]) Seja $\mathbb{K} u m$ sgn. Então

(6.1) $\min \{c(B) \mid B$ é uma cobertura de $P$, boa para $\check{P}\}$

$=\max \left\{\sum_{u \in P} y(u) \mid y \geqslant 0 ; \sum_{u \in P} y(u) \chi^{\rho u} \leqslant c\right\}$.

Lema 6.2 Seja $\mathbb{K}$ um sgn, $e \mathrm{P}^{\uparrow} \mathbb{K}:=\mathrm{P} \mathbb{K}+\mathbb{R}_{+}^{E}$.

Então $\mathrm{P}^{\uparrow} \mathbb{K}$ é igual ao conjunto $Q$ dos $x \in \mathbb{R}^{E}$ tais que

(i) $x \geqslant 0, e$

(ii) $x(\rho u) \geqslant 1$ para cada $u \in P$.

Prova. É claro que $\mathrm{P}^{\uparrow} \mathbb{K} \subseteq Q$. Suponha, por contradição, que $Q \backslash \mathrm{P}^{\uparrow} \mathbb{K} \neq \emptyset$. Seja $z \in Q \backslash \mathrm{P}^{\uparrow} \mathbb{K}$. Pelo Teorema do Hiperplano Separador, existem $c \in \mathbb{R}^{E}$ e $\eta \in \mathbb{R}$ tais que

$$
c z=\eta \quad \mathrm{e} \quad x \in \mathrm{P}^{\uparrow} \mathbb{K} \text { implica } c x>\eta,
$$

o que, por sua vez, acarreta em $\min \{c x \mid x \in Q\}<\min \left\{c x \mid x \in \mathrm{P}^{\uparrow} \mathbb{K}\right\}$. É claro que $c \geqslant 0$ pois, caso contrário, o mínimo nas duas expressões é $-\infty$. Note que o dual de $\min \{c x \mid x \in Q\}$ é 


$$
\max \left\{\sum_{u \in P} y(u) \mid y \geqslant 0, \sum_{u \in P} y(u) \chi^{\rho u} \leqslant c\right\} .
$$

Agora, aliando-se o Teorema Fraco da Dualidade com o Teorema 6.1, obtemos a seguinte contradição:

$$
\begin{aligned}
& \min \{c x \mid x \in Q\} \\
& \geqslant \max \left\{\sum_{u \in P} y(u) \mid y \geqslant 0 ; \sum_{u \in P} y(u) \chi^{\rho u} \leqslant c\right\} \\
& =\min \{c(B) \mid B \text { é uma cobertura de } P, \text { boa para } \check{P}\} \\
& \geqslant \min \left\{c x \mid x \in \mathrm{P}^{\uparrow} \mathbb{K}\right\},
\end{aligned}
$$

o que estabelece o lema.

Teorema 6.3 Seja $\mathbb{K}$ um sgn. Então $\mathrm{P} \mathbb{K}$ é igual ao conjunto $Q$ dos $x \in \mathbb{R}^{E}$ tais que

$$
\begin{aligned}
& \text { (i) } 0 \leqslant x \leqslant 1, \\
& \text { (ii) } x(\rho u) \geqslant 1 \text { para cada } u \in P \text {, } \\
& \text { (iii) } x(\rho u)=1 \text { para cada } u \in \check{P} \text {. }
\end{aligned}
$$

Ademais, $\operatorname{dim} \mathrm{PKK} \leqslant|E|-|\check{P}|$.

Prova. Seja $H:=\left\{x \in \mathbb{R}^{E} \mid x(\rho u)=1\right.$ para cada $\left.u \in \check{P}\right\}$. Vamos provar que $\mathrm{P} \mathbb{K}=\mathrm{P}^{\uparrow} \mathbb{K} \cap H$ o que, combinado com o Lema 6.2, estabelece este teorema. É claro que $\mathrm{P} \llbracket \mathrm{K} \subseteq \mathrm{P}^{\uparrow} \mathbb{K} \cap H$. Para a recíproca, seja $x \in \mathrm{P}^{\uparrow} \cap H$. Denote por $\mathcal{B}$ o conjunto das coberturas de $P$, boas para $\check{P}$. Tome $\xi: \mathcal{B} \rightarrow[0,1]$ tal que

$$
\begin{aligned}
& \text { (i) } \xi \geqslant 0 \text { e } \sum\left[\xi_{B} \mid B \in \mathcal{B}\right]=1, \\
& \text { (ii) } y:=\sum\left[\xi_{B} \chi^{B} \mid B \in \mathcal{B}\right] \leqslant x \text {, e } \\
& \text { (iii) } \sum\left[\xi_{B}|B| \mid B \in \mathcal{B}\right] \text { é máximo. }
\end{aligned}
$$

Um tal $\xi$ existe pois o conjunto sobre o qual maximizamos é compacto e a função a maximizar é contínua. Note que $y \in \mathrm{P} \mathbb{K}$ devido a (6.4.i) e (6.4.ii). Afirmamos que $y=x$. Suponha que este não é o caso. Defina $z:=x-y$. Obviamente, $y+z \in \mathrm{P}^{\uparrow} \cap H$. Então $z(e)>0$ para algum $\operatorname{arco} e \operatorname{em} E$.

Fato 6.3.1 Para cada $m \in \check{P}: e$ evita $m$.

- Suponha, por contradição, que existe $m \in \check{P}$ tal que $e \in \rho m$. Temos, então, a seguinte contradição:

$$
1>y(\rho m)=\sum_{B \in \mathcal{B}} \xi_{B} \chi^{B}(\rho m)=1 .
$$

A primeira desigualdade é válida pois $e \in \rho m$ e $z(e)>0$; a segunda, pois $y \in \mathrm{P} \mathbb{K}$; e a última, pois $B$ é uma cobertura de $P$ boa para $\check{P}$ (e daí $\left.\chi^{B}(\rho m)=1\right)$, e $\sum_{B \in \mathcal{B}} \xi_{B}=1$.

Ora, $x(e) \leqslant 1$ e $z(e)>0$ implicam que $y(e)<1$ donde existe $F \in \mathcal{B}$ tal que $e \notin F$. Seja, agora, $F^{\prime}:=F \cup\{e\}$. Note que $F^{\prime}$ é uma cobertura de $P$ boa para $\check{P}$ pois, pelo Fato 6.3.1 $e$ evita cada um dos núcleos em $\check{P}$. Há dois casos a considerar. Se $z(e) \geqslant \xi_{F}$, então definimos 
$\triangleright \quad \xi^{\prime}:=\xi\left[F \mapsto 0, F^{\prime} \mapsto \xi_{F^{\prime}}+\xi_{F}\right]$.

Se, por outro lado, $z(e)<\xi_{F}$, então definimos

$\triangleright \quad \xi^{\prime}:=\xi\left[F \mapsto \xi_{F}-z(e), F^{\prime} \mapsto \xi_{F^{\prime}}+z(e)\right]$.

Agora, $\xi^{\prime} \geqslant 0, \sum\left[\xi_{B}^{\prime} \mid B \in \mathcal{B}\right]=1$ e $\sum\left[\xi_{B}^{\prime} \chi^{B} \mid B \in \mathcal{B}\right] \leqslant x$. Ademais,

$$
\sum\left[\xi_{B}^{\prime}|B| \mid B \in \mathcal{B}\right]>\sum\left[\xi_{B}|B| \mid B \in \mathcal{B}\right]
$$

e esta contradição mostra que $z=0 \mathrm{e}$, portanto, $y=x$, como queríamos.

\subsection{A integralidade de um certo poliedro supermodular}

Nesta seção, vamos estabelecer a integralidade de um certo poliedro envolvendo os sistemas generalizados de núcleos com a propriedade do cruzamento, usando uma técnica de Jain [Jai01], extensivamente ilustrada em Lau, Ravi and Singh [LRS11]. Destacamos que, como os sistemas generalizados de núcleos com a propriedade do cruzamento generalizam famílias intersectantes de biconjuntos, o Teorema 6.5, que segue, inclui como caso especial a polinomialidade do problema do subdigrafo enraizado $k$-conexo de custo mínimo [LRS11]. Em particular, este teorema generaliza o Teorem 6.2.8 in [LRS11]. Ressaltamos que a prova que exibiremos induz, módulo um oráculo de separação, um algoritmo polinomial para o problema da minimização.

Seja $\mathbb{K}$ um sgnc. Uma função $p: P \rightarrow \mathbb{R}$ é uma função supermodular nos membros positivos $e$ intersectantes se para cada $u, w \in p^{+}$:

$$
u \bowtie w \text { implica } p(u)+p(w) \leqslant p(u \vee w)+p(u \wedge w) .
$$

Seja $\mathbf{S}_{p}$ o sistema de inequações:

(i) $0 \leqslant x \leqslant 1$, e

(ii) $x(\rho u) \geqslant p(u)$ para cada $u \in P$,

onde $x \in \mathbb{R}^{E}$. Vamos abusar um pouco da notação e confundir o sistema $\mathbf{S}_{p}$ com o conjunto dos $x$ que satisfazem cada restrição em $\mathbf{S}_{p}$. Assim, escrevemos $x \in \mathbf{S}_{p}$ se $x$ satisfaz (6.6).

É conveniente também introduzir a seguinte notação. Para cada $x \in \mathbf{S}_{p}$, seja eq $\left(x, \mathbf{S}_{p}\right)$ o conjunto das inequações de $\mathbf{S}_{p}$ que $x$ satisfaz com igualdade, isto é,

$$
\text { eq }\left(x, \mathbf{S}_{p}\right):=\left\{\chi^{\{a\}} \mid a \in E, x(a) \in\{0,1\}\right\} \bigcup\left\{\chi^{\rho u} \mid u \in P, x(\rho u)=p(u)\right\} .
$$

Vamos precisar de um resultado elementar de teoria dos poliedros, veja, por exemplo, Lau, Ravi and Singh [LRS11], ou a obra ímpar de Schrijver [Sch86].

Lema 6.4 Seja $M$ um conjunto finito, $\left\{a_{i} \mid i \in M\right\} \subseteq \mathbb{R}^{m}, b \in \mathbb{R}^{M}$ e $Q:=\left\{x \in \mathbb{R}^{n} \mid a_{i} x \geqslant b_{i} ; x \geqslant 0\right\}$ um poliedro. Se x é um ponto extremo de $Q$ e $x>0$, então rank $\left\{a_{i} \mid a_{i} x=b_{i}, i \in M\right\}=n$.

Teorema 6.5 Seja $\mathbb{K}$ um sgn-c não-trivial e $p: P \rightarrow \mathbb{Z}$ uma função supermodular intersectante nos membros positivos. Então o poliedro $\mathbf{S}_{p}$ é inteiro. 
Prova. Para cada $x \in \mathbf{S}_{p}$, seja $T^{x}:=\left\{u \in p^{+} \mid x(\rho u)=p(u)\right\}$. Observe que $\mathbf{S}_{p} \neq \emptyset$ pois $\mathbb{K}$ é não-trivial.

Fato 6.5.1 Se $x \in \mathbf{S}_{p}$ e $x>0$ então

(i) $T^{x}$ é $\bowtie$-fechado.

(ii) para cada $u, w \in T^{x}$ :

$u \bowtie w$ implica $x(\rho u)+x(\rho w)=x(\rho(u \vee w))+x(\rho(u \wedge w))$.

- A prova deste fato é bem rotineira. Seja $x \in \mathbf{S}_{p}$. Sejam $u, w \in T^{x}$ e suponha que $u \bowtie w$. Então

$$
\begin{aligned}
& p(u)+p(w) \\
& =x(\rho u)+x(\rho w) \\
& \geqslant x(\rho(u \vee w))+x(\rho(u \wedge w)) \\
& \geqslant p(u \vee w)+p(u \wedge w) \\
& \geqslant p(u)+p(w),
\end{aligned}
$$

onde a primeira igualdade segue de $u, w \in T^{x}$; a primeira desigualdade, por submodularidade; a segunda desigualdade, de $x \in \mathbf{S}_{p}$; a terceira desigualdade, de $u, w \in p^{+}$e da supermodularidade de $p$. Vale, portanto, a igualdade em todas as passagens e daí

$$
x(\rho(u \vee w))=p(u \vee w) \quad \text { e } \quad x(\rho(u \wedge w))=p(u \wedge w) .
$$

Como $x>0$ e $\mathbb{K}$ é não-trivial, então $p(u \vee w)>0$ e $p(u \wedge w)>0$, e isto estabelece (i) e (ii).

Lembre-se que para uma parte $X$ de $P$, span $X$ denota o conjunto das combinações lineares de $\left\{\chi^{\rho u} \mid u \in X\right\}$.

Fato 6.5.2 Seja $x \in \mathbf{S}_{p} \operatorname{com} x>0$, e $L \subseteq T^{x}$.

Se $L$ é um subconjunto laminar maximal de $T^{x}$ então $\operatorname{span} L=\operatorname{span} T^{x}$.

- Suponha, por contradição, que span $L \subset \operatorname{span} T^{x}$. Seja $X:=\left\{u \in T^{x} \mid \chi^{\rho u} \notin \operatorname{span} L\right\}$. Como $T^{x}$ é $\bowtie$-fechado e $X \neq \emptyset$ então, pelo Lema 2.1, existe $u \in X$ tal que

(*) $\quad$ para cada $\ell \in L_{u}, z \in T^{x}:$ se $z \prec \ell$, então $\chi^{\rho z} \in \operatorname{span} L$.

Além disso, o Lema 3.1 acarreta na existência de uma $u$-spi f para a qual

$$
u \vee \mathbf{f} \in T^{x} \quad \text { e } \quad L \cup\{u \vee \mathbf{f}\} \text { é laminar. }
$$

A maximalidade de $L$ implica $u \vee \mathbf{f} \in L$. Agora, de $x>0$ e do Fato 6.5.1 vem, usando o Lema 2.3iii, que para cada $v, w \in T^{x}:$

$(* *) \quad v \bowtie w$ implica $\chi^{\rho v}+\chi^{\rho w}=\chi^{\rho(v \vee w)}+\chi^{\rho(v \wedge w)}$.

Fato 6.5.2.1 $\chi^{\rho u}-\chi^{\rho(u \vee \mathbf{f})} \in \operatorname{span} L$.

-• A prova é uma indução no comprimento de f. Note que $u \notin L$ implica que o comprimento de $\mathbf{f}$ é ao menos 1. Suponha, primeiro, que $\mathbf{f}=\ell$. Então, em virtude de $(* *)$ e $u \bowtie \ell$, temos que 


$$
\chi^{\rho u}-\chi^{\rho(u \vee \ell)}=\chi^{\rho(u \wedge \ell)}-\chi^{\rho \ell} .
$$

No entanto, $(*)$ implica que $\chi^{\rho(u \wedge \ell)} \in \operatorname{span} L$ o que, aliado à $\chi^{\rho \ell} \in \operatorname{span} L$, fornece

$$
\chi^{\rho u}-\chi^{\rho(u \vee \ell)} \in \operatorname{span} L .
$$

Suponha, agora, que o comprimento de $\mathbf{f}$ é maior que 1 . Seja, então, $\mathbf{f}^{\prime}$ e $\ell$ tais que $\mathbf{f}=\mathbf{f}^{\prime} \cdot m$. Por hipótese de indução, segue que $\chi^{\rho u}-\chi^{\rho\left(u \vee \mathbf{f}^{\prime}\right)} \in \operatorname{span} L$. Ora, $u \vee \mathbf{f}^{\prime} \bowtie \ell \mathrm{e}(* *)$ acarretam

$$
\chi^{\rho\left(u \vee \mathbf{f}^{\prime}\right)}-\chi^{\rho(u \vee \mathbf{f})}=\chi^{\left.\rho\left(u \vee \mathbf{f}^{\prime}\right) \wedge \ell\right)}-\chi^{\rho \ell} .
$$

Mais uma vez, $(*)$ fornece $\chi^{\rho\left(\left(u \vee \mathbf{f}^{\prime}\right) \wedge \ell\right)} \in \operatorname{span} L$ o que, quando combinado com $\chi^{\rho \ell} \in \operatorname{span} L$, implica que $\chi^{\rho\left(u \vee \mathbf{f}^{\prime}\right)}-\chi^{\rho(u \vee \mathbf{f})} \in \operatorname{span} L$. Logo, $\chi^{\rho u}-\chi^{\rho(u \vee \mathbf{f})} \in \operatorname{span} L$, pois

$$
\chi^{\rho u}-\chi^{\rho(u \vee \mathbf{f})}=\chi^{\rho u}-\chi^{\rho\left(u \vee \mathbf{f}^{\prime}\right)}+\chi^{\rho\left(u \vee \mathbf{f}^{\prime}\right)}-\chi^{\rho(u \vee \mathbf{f})} .
$$

e isto conclui a prova do fato.

Como $u \vee \mathbf{f} \in L$, então $\chi^{\rho(u \vee \mathbf{f})} \in \operatorname{span} L$ o que, combinado com $\chi^{\rho u}-\chi^{\rho(u \vee \mathbf{f})} \in \operatorname{span} L$, implica que $\chi^{\rho u}$ está também em $\operatorname{span} L$, o que é uma contradição. Isto completa a prova do fato.

A próxima afirmação é essencialmente uma reformulação do Lema 6.2.10 em [LRS11]. Para enunciá-la, necessitamos de algumas definições. Seja $X$ um subconjunto de $P$. Dizemos que uma função $f: X \rightarrow 2^{E}$ é uma distribuição de $E$ sobre $X$ se

(i) para cada $u, w \in X: u \neq w$ implica $f(u) \cap f(w)=\emptyset$,

(ii) para cada $u \in X: f(u) \neq \emptyset$, e

(iii) para cada $u \in X: u$ maximal em $X$ implica $|f(u)| \geqslant p(u)+1$.

Fato 6.5.3 Seja $x \in \mathbf{S}_{p}$, e seja $L$ uma parte laminar de $T^{x}$ tal que $L$ é linearmente independente. Se $0<x<1$ então existe uma distribuição $f: L \rightarrow 2^{E}$ de $E$ sobre $L$.

- A prova é uma indução em $|L|$. Se $L=\emptyset$, não há nada a provar. Suponha, pois, $L \neq \emptyset$. Seja $\ell$ um núcleo maximal em $L$ e $L^{\prime}:=L \backslash\{\ell\}$. Por hipótese de indução, existe uma distribuição $f^{\prime}: L^{\prime} \rightarrow 2^{E}$ de $E$ sobre $L^{\prime}$. Suponha, primeiro, que $\ell$ é também um núcleo minimal em $L$. Ora, $\rho \ell \neq \emptyset$ e $x>0$ acarretam $x(\rho \ell)>0$; ademais, $x \in \mathbf{S}_{p}$ fornece $x(\rho \ell) \geqslant p(\ell)$. Aliando-se estas últimas observações $\operatorname{com} x<1$, obtemos $d(\ell) \geqslant p(\ell)+1$. Note que $\rho m \cap \rho \ell=\emptyset$ para cada $m \in L$, caso contrário, $m \bowtie \ell$ contraria a laminaridade de $L$. Defina $f: L \rightarrow 2^{E}$ pondo-se

$\triangleright \quad f:=f^{\prime}[\ell \mapsto \rho \ell]$

Está claro que $f$, neste caso, é uma distribuição de $E$ sobre $L$.

Suponha, agora, que $\ell$ não é minimal em $L$. Seja $M$ o conjunto dos núcleos $m \in L$ maximais tais que $m \prec \ell$. Então $\left|f^{\prime}(m)\right| \geqslant p(m)+1$ para cada $m \in M$. Selecione, para cada $m \in M$, um núcleo, digamos $\eta_{m}$, em $f^{\prime}(m)$. Ponha $F:=\bigcup\left\{f^{\prime}(m) \backslash\left\{\eta_{m}\right\} \mid m \in M\right\}$. Se $|F|>p(\ell)$, então defina $f: L \rightarrow 2^{E}$ pondo-se

$\triangleright \quad f:=f^{\prime}\left[\ell \mapsto F, M \ni m \mapsto f^{\prime}(m) \backslash\left\{\eta_{m}\right\}\right]$. 
Claramente, $f$ é uma distribuição de $E$ sobre $L$ e, neste caso, não há mais nada a provar.

Seja $B:=\rho \ell \backslash\left(\bigcup_{m \in M} \rho m\right)$. Suponha, agora, que $|F| \leqslant p(\ell)$. Note que $|F| \geqslant \sum\left[p^{\prime}(m) \mid m \in M\right]$.

Fato 6.5.3.1 $B \neq \emptyset$.

•• Suponha, por contradição, que $B=\emptyset$. Então $\rho \ell \subseteq \bigcup_{m \in M} \rho m$ donde, como $x>0$, segue que

$$
x(\rho \ell) \leqslant \sum[x(\rho m) \mid m \in M] \leqslant|F| \leqslant p(\ell)=x(\rho \ell) .
$$

Assim, vale a igualdade em todas as passagens o que, aliado a $x>0$, fornece $\chi^{\rho \ell}=\sum\left[\chi^{\rho m} \mid m \in M\right]$. Isto contraria a independência linear de $L$.

Ora, $x(B) \geqslant p(\ell)-|F|$ pois, caso contrário, se $x(B)<p(\ell)-|F|$ então temos a seguinte contradição.

$$
p(\ell)=x(\rho \ell) \leqslant x(B)+\sum[x(\rho m) \mid m \in M]<p(\ell)-|F|+|F|=p(\ell) .
$$

Observe que $|B| \geqslant p(\ell)-|F|+1$. De fato, se $p(\ell)=|F|$, então a desigualdade é evidente pois $B \neq \emptyset$. Se $p(\ell)>|F|$ então a desigualdade segue de $x<1$.

Fato 6.5.3.2 Para cada $e \in B, u \in L^{\prime}: e \notin \rho u$.

- Suponha, por contradição, que existam $e \in B$ e $u \in L^{\prime}$ tais que $e \in \rho u$. Então, em virtude de $(\rho .3)$, vem que $u \widetilde{\bowtie \ell}$. Como $L$ é laminar, segue que $u \sim \ell$. No entanto, $\ell$ é maximal em $L$ e, assim, $u \prec \ell$. Além disso, existe $m \in M$ tal que $u \preccurlyeq m \prec \ell$ e $(\rho .1)$ implica $e \in \rho m$, uma contradição.

Agora, defina $f: L \rightarrow 2^{E}$ pondo-se

$$
\triangleright \quad f:=f^{\prime}\left[\ell \mapsto F \cup B, M \ni m \mapsto f^{\prime}(m) \backslash\left\{\eta_{m}\right\}\right]
$$

É fácil verificar que $f$ é uma distribuição de $E$ sobre $L$, completando, assim, a prova do fato.

Fato 6.5.4 Seja $x$ um ponto extremo de $\mathbf{S}_{p}$. Então existe $a \in E$ tal que $x(a) \in\{0,1\}$.

- Suponha, por contradição, que $0<x<1$. Pelo Lema 6.4, temos que $|E|=|L|$. Seja $f$ uma distribuição de $E$ sobre $L$ garantida pelo Fato 6.5.3. Seja $m$ um núcleo maximal em $L$. Então, em virtude de (6.7.iii), $|f(m)| \geqslant p(m)+1$. Agora, $0<x(\rho m)=p(m)$ e, portanto, $f(m) \geqslant 2$ o que, quando aliado a (6.7.i) e (6.7.ii), acarreta $|E|>|L|$, o que é uma contradição. Concluímos, assim, que existe $a \in E$ tal que $x(a) \in\{0,1\}$.

Seja $x$ um ponto extremo de $\mathbf{S}_{p}$. Então existe $a \in E$ tal que $x(a) \in\{0,1\}$. Suponha, primeiro, que $x(a)=0$. Vamos, por este caso ser trivial, argumentar informalmente. Considere o sgn-c obtido de $\mathbb{K}$ através da remoção do arco $a$ de cada $u \in P$ com $a \in \rho u$ e $E^{\prime}:=E \backslash\{a\}$. A restrição, $x^{\prime}$, de $x$ ao conjunto $E^{\prime}$ é claramente um ponto extremo do poliedro correspondente e, portanto, por indução, $x^{\prime}$ é inteiro. Logo, $x$ também é inteiro.

Agora, suponha que $x(a)=1$. Defina $p^{\prime}: P \rightarrow \mathbb{R}$ pondo-se para cada $u \in P$ :

$$
p^{\prime}(u):=\text { se } a \in \rho u \text { então } p(u)-1 \text {, senão } p(u) .
$$


Note que $p^{\prime}$ é supermodular nos membros positivos e intersectantes uma vez que

- $p^{\prime}=p-d_{\{a\}}$

- $p$ é supermodular nos membros positivos e intersectantes, e

- $d_{\{a\}}$ é submodular nos membros intersectantes.

Defina, também, $x^{\prime}: E \rightarrow \mathbb{R}_{+}$pondo-se para cada $e \in E$ :

$$
x^{\prime}(e):=\text { se } a=e \text { então } x(a)-1, \text { senão } x(a) .
$$

Como $x$ é um ponto extremo de $\mathbf{S}_{p}$, então $x^{\prime}$ é um ponto extremo de $\mathbf{S}_{p^{\prime}}$. Para ver isso, apenas observe que eq $\left(x, \mathbf{S}_{p}\right)=$ eq $\left(x^{\prime}, \mathbf{S}_{p^{\prime}}\right)$. Assim, por hipótese de indução, $x^{\prime}$ é inteiro e, por conseguinte, $x$ também é inteiro. 


\section{Capítulo 7}

\section{Considerações finais}

Vamos resumir brevemente as nossas contribuições neste trabalho. Estendemos as ideias de submodularidade de Lovász e Frank para os sistemas generalizados de núcleos com uma família mista, que constituem o arcabouço mais geral conhecido onde estas ideias podem ser aplicadas. Provamos uma série de teoremas neste arcabouço - envolvendo tanto empacotamentos inteiros quanto fracionários - que melhoram, quando instanciados para o problema do empacotamento de arborescências e de ramificações, os resultados conhecidos. Finalmente, também mostramos que é possível resolver, em tempo polinomial, módulo um oráculo de separação, o problema de encontrar uma cobertura mínima de um sistema generalizado de núcleos sujeito a restrições supermodulares. 
74 CONSIDERAÇÕES FINAIS 


\section{Referências Bibliográficas}

[Bar04] F. Barahona. Fractional packing of $t$-joins. SIAM Journal of Discrete Mathematics, $17: 661-669,2004.7$

[BF08] K. Bérczi e A. Frank. Variations for lovász submodular ideas. Em G.O.H. Katona M. Grötschel, editor, Building Bridges Between Mathematics and Computer Science, páginas 137-164. $1^{\circ}$ edição, 2008. 3, 8, 13, 37, 46

[Edm73] J. Edmonds. Edge-disjoint branchings. Em Combinatorial Algorithms, páginas 91-96. $1^{\circ}$ edição, 1973. 2, 6, 8, 57

[Fra79] A. Frank. Kernel systems of directed graphs. Acta Scientiarum Mathematicarum (Szeged), 41 (1-2):63-76, 1979. 2, 3, 13, 18

[Fra99] A. Frank. Increasing the rooted-connectivity of a digraph by one. Mathematical Programming, 84:565-576, 1999. 3, 13, 16, 65

[Fra09] A. Frank. Rooted $k$-connections in digraphs. Discrete Applied Mathematics, 157:12421254, 2009. 3, 6

[Fra11] A. Frank. Connection in combinatorial optimization. Oxford University Press, $1^{\mathrm{o}}$ edição, 2011. 2

[GM98] H.N. Gabow e K.S. Manu. Packing algorithms for arborescences (and spanning trees) in capacitated graphs. Mathematical Programming, 82:83-109, 1998. 6, 7, 33, 47, 57

[Jai01] K. Jain. A factor 2 approximation algorithm for the generalized steiner network problem. Combinatorica, 21:39-60, 2001. 67

[Lov76] L. Lovász. On two minimax theorems on graph theory. Journal of Combinatorial Theory, Series B, 21:96-103, 1976. 3, 13, 18

[LRS11] L. C. Lau, R. Ravi e M. Singh. Iterative methods in combinatorial optimization. Cambridge University Press, $1^{\circ}$ edição, 2011. 67, 69

[Mat12] Y. Matsuoka. Fractional packing in ideal clutters. Mathematical Programming, 133:159-169, 2012. 7

[Sch86] A. Schrijver. Theory of Linear and Integer Programming. Wiley, $1^{\circ}$ edição, 1986. 67

[Sch03] A. Schrijver. Combinatorial Optimization: Polyhedra and Efficiency 3 vols. Springer, $1^{\circ}$ edição, 2003. 8, 47, 58

[Sze01] L. Szegó. Note on covering intersecting set-systems by digraphs. Discrete Mathematics, 234:187-189, 2001. 3, 8, 13, 37, 46 


\section{Índice Remissivo}

$\wedge$-fechada, 14

$\bowtie$-fechada, 14

$r$-arborescência, 2

st-caminho, 2

iposet, 14

pim, 37

sbi, 4

grau, 4

sgn-c-fm, 47

sgnmf

inteiro, 37

sgn

não-trivial, 16

afim-independente, 24

arbóreo, 18

arco, 2

cabeça, 2

cauda, 2

arcos, 15

biconjunto, 3

biconjuntos

intersectante, 4

boa, 18

capacidade, 26, 41

cobertura, 1, 18

cobre, 4, 18

comparável, 13

digrafo, 2

distribuição, 69

empacota, 6, 38

empacotamento, 37, 38

inteiro, 38

entra, 4

especial, 26, 41

evita, 18

família, 9 família mista, 37

intersectante, 2, 14

laminar, 14

legal, 48

núcleo, 13

não-capacitado, 16

politopo das coberturas, 65

pontas, 2

poset, 13

maximal, 13

minimal, 13

positivo, 22

prefixo, 10

propriamente intersectantes, 13

propriedade da intersecção mista, 37

ramificação, 8

sequência, 10

vazia, 10

sequência propriamente intersectante, 32

sequência vazia, 31

sistema generalizado de núcleos, 16 inteiro, 16

sistema generalizado de núcleos com uma família mista, 37

sistemas de biconjuntos intersectantes, 4

supermodular

membros positivos e intersectantes, 67

suporte, 10

vértice, 2 